
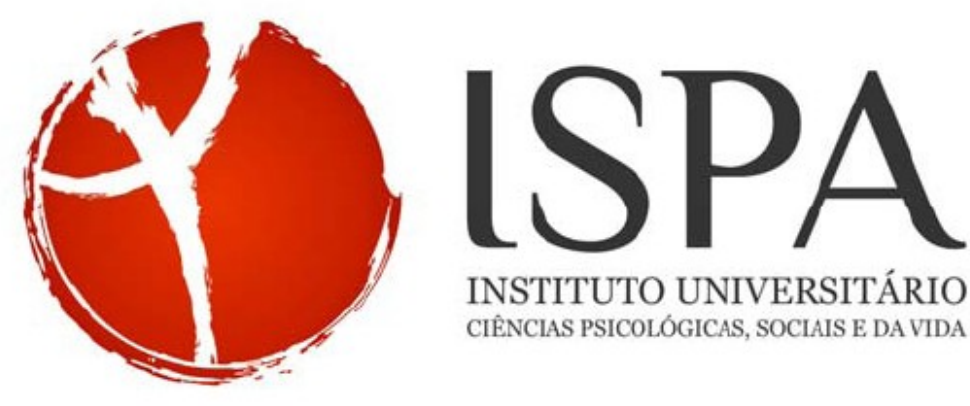

INSTITUTO UNIVERSITÁRIO

CIÊNCIAS PSICOLÓGICAS, SOCIAIS E DAVIDA

\title{
FAMILIARITY, CHALLENGE AND PROCESSING OF PERSUASION MESSAGES
}

Ricardo Jorge Rodrigues Moita da Fonseca

\author{
Tese orientada por \\ Proferssora Doutora Teresa Garcia-Marques \\ ISPA - Instituto Universitário \\ $\mathrm{e}$ \\ co-orientada por \\ $\mathrm{PhD}$. James Blascovich \\ University of California, Santa Barbara
}

Tese submetida como requisito parcial para obtenção do grau de

Doutoramento em Psicologia

Área de especialidade. Psicologia Social 
Tese apresentada para cumprimento dos requisitos necessários à obtenção do grau de Doutor em Psicologia na área de especialização de Psicologia Social realizada sob a orientação de Teresa Garcia-Marques e co-orientação de James Blascovich, apresentada no ISPA - Instituto Universitário no ano de 2012.

O presente trabalho foi financiado pelo Programa Operacional Ciência e Inovação (POCI 2010) da Fundação para a Ciência e Tecnologia (SFRH/BD/30515/ 2006).

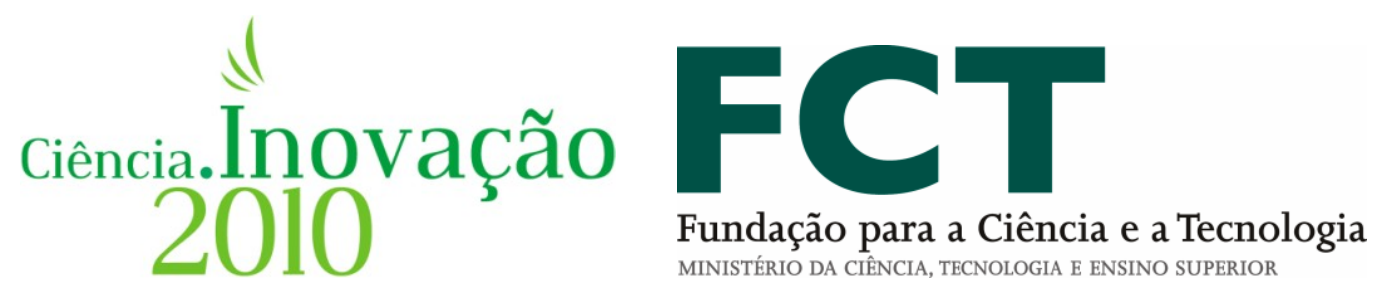




\section{Acknowledgments}

Well, it has been a long journey since the beginning of this dissertation. I have learned so much and experienced so many things throughout this process that I am afraid I have no words to express my gratitude to all the people involved in it. But here is an honest and brief attempt.

Firstly, I would like to thank FCT for the financial support which made the accomplishment of this dissertation possible. Secondly, but most important, I would like to thank both my advisors. Teresa, thank you for teaching me all you know. Thank you for putting so much effort and vision on this dissertation. Thank you for standing by me even when the times were rough and for giving me all the "frustrations" that I needed to become a stronger researcher. I am sure that, without your dedication, this dissertation would not have the quality it has. Jim, thank you for teaching me all you know. Thank you for the way you receive me at UCSB. It is not easy to leave your country and go to the other side of the planet, but you sure made it easier. I learned a lot in your labmeetings. I am sure I am a more complete researcher because of your advices and teachings.

For all the emotional support, I also would like to thank my parents, my big brother, my sister and my aunt. And Carlos. And Ricardo. It was surely important to lie on your shoulders when the times got tough. Words are not enough to express my gratitude for your love.

I also would like to thank my colleagues for their inputs and friendship. Mariana, Ana, Pedro, Rita, Alexandre, Cristina, Cátia, Inês and Hugo. Also, Tânia, Sara, Sofia, Rita J., André, Rui, Margarida, Ludmila, David K. And my dearests David and Marilia. To my fellow American colleagues: Deb, Cade, Masaki, Jane, Christena, Cynthia and Debbie and all the research assistants, particularly Natalya.

To ISPA-IU, in particular to UIPCDE. Thank you to Ana Cristina Quelhas, Jorge Senos, Csongor Juhos and a special word to Maria Gouveia-Pereira responsible for making me fall in love with social facilitation. Outside ISPA, I also would like to thank all the other professors, both from Portugal and USA, who offered me their insights throughout the PhD: Mario Ferreira, Leonel Garcia-Marques, Diane Mackie, and Brenda Major. On this note, I also take the opportunity to thank all the dead ones who have inspired me, especially Stanley Milgram and Robert Zajonc. 
To all my friend who stood there for me and had the patience for taking all the "sorryI-can't-meet you-I'm-doing-phd-stuff'.

Dona Mécia de Sena for all the love you gave me while I was in a far place. I am such a more complete person after metting you.

Lastly, Mother, wheverever you are, your love made me the strong person that I am today. This one is for you. Hope you are proud. 
Palavras-chave:

Familiaridade; desafio e ameaça; modos de processar; presença de outros.

Key words:

Familiarity; challenge and threat; modes of processing; presence of others.

\section{Categorias de Classificação da tese}

PsycINFO Classification Categories and Codes:

2300 Human Experimental Psychology 2340 Cognitive Processes

2343 Social Psychology 2360 Motivation and Emotion

3000 Social Psychology 3040 Social Perception and Cognition 


\begin{abstract}
This thesis investigates the relationship between an experience of familiarity and a motivational state of challenge with how information is processed in a persuasion context.

Previous research on social cognition has suggested that familiarity not only impacts a wide range of cognitive processes, but also regulates the activation of a more analytic information-processing mode, an assumption of the Familiarity of As a Regulation Mechanism model (Garcia-Marques, 1999; Garcia-Marques et al., 2010). On a different field, research on the Biopsychosocial model of challenge and threat (Blascovich et al., 1993, 1999) has suggested that familiarity influences the activation of a motivational state of challenge. These two approaches suggest, therefore, that an experience of familiarity is able to influence both cognitive and motivational processes features. The overlap between the assumptions underlying both approaches is here explored being suggested the possibility that they might be closely related. For example, both approaches assume that an experience of familiarity signals individuals with necessary resources available and accessible in memory to deal with the situation. In this thesis, we have explored the relationship between these two approaches developing four experiments that could simultaneously inform about information-processing modes and assess the cardiovascular responses that typically map the motivational state.

Experiment 1 showed the expected association of familiarity with non-analytical processing and at the same time the exhibition of a challenge type of cardiovascular responses. Interestingly these two effects that were activated by the same source, familiarity, did not seem to be related. Neither the observed cardiovascular indexes explained why individuals engaged in less analytic processing, nor did this processing mode was associated with the cardiovascular indexes. To continue exploring the relationship between these two effects, experiment 2 tested if the motivational state of challenge could promote less analytic processing by itself. Although the manipulation of motivational challenge did in fact influence how information was processed and was associated with the correspondent cardiovascular pattern of challenge, once again, the cardiovascular indexes were not related with the cognitive effect.

The subsequent studies were designed to directly test the observed independence of both processes. We hypothesized that this observed dissociation could be in some way related with the fact that both processes depend on different levels of task-engagement. Experiment 3 replicates experiment 2 by manipulating the motivational state of challenge and adding to it a manipulation of task-engagement (presence versus absence of an observer). Results revealed that the two previously observed effects were only found in the task-engagement condition (i.e. in the presence of the observer). In experiment 4, we went back to the original study of the experience of familiarity and thus replicated experiment 1, adding to it the same manipulation of task-engagement. Results revealed that although the motivational effects disappeared in the low engagement condition (i.e. those who were alone), the cognitive impact was always observed regardless of the task-engagement level. To our view, these results are suggesting that the two effects here approached - the cognitive and motivational impact of familiarity, are related indeed. However, they are related under specific conditions, for example, the degree with which individuals are engaged with the task. As such, we claim that their co-occurrence does not mean that they are part of the same process. This assumption is discussed and a set of new experiments is proposed to further support it.
\end{abstract}

Keywords: Familiarity; challenge and threat; modes of processing; presence of others. 
Index

Overview 1

Section I 3

Chapter I: Familiarity, Cognitive Processing and Motivational States 5

The impact of familiarity on cognitive processing 5

The impact of familiarity on motivational states of challenge and threat 12

Chapter II: Hypothesizing the relationship between the FARM and the BPS 25 model

Familiarity and Challenge $\quad 25$

Overview of the Experiments $\quad 28$

Section II: Empirical Section 33

Experiment 1. $\quad 35$

$\begin{array}{ll}\text { Method } & 37\end{array}$

Participants and Design $\quad 37$

$\begin{array}{ll}\text { Material } & 37\end{array}$

Procedure 38

$\begin{array}{ll}\text { Dependent Measures } & 42\end{array}$

Results and Discussion $\quad 43$

Experiment 2. $\quad 52$

Method 53

Participants and Design 53

Material 53

Procedure $\quad 54$

Dependent Measures $\quad 56$

Results and Discussion $\quad 57$

Experiment 3. $\quad 62$

Method $\quad 63$

Participants and Design $\quad 63$

$\begin{array}{ll}\text { Material } & 63\end{array}$

Procedure $\quad 63$

$\begin{array}{ll}\text { Dependent Measures } & 65\end{array}$

Results and Discussion $\quad 65$

Experiment 4. 70

$\begin{array}{ll}\text { Method } & 70\end{array}$

$\begin{array}{ll}\text { Partcipants and Design } & 70\end{array}$

$\begin{array}{ll}\text { Material } & 71\end{array}$

$\begin{array}{ll}\text { Procedure } & 71\end{array}$

$\begin{array}{ll}\text { Dependent Measures } & 72\end{array}$

$\begin{array}{ll}\text { Results and Discussion } & 73\end{array}$ 
General Discussion

References

\section{Appendixes}

$\begin{array}{ll}\text { Appendix A: Persuasive messages } & 100\end{array}$

$\begin{array}{ll}\text { Appendix B: Pre-attitudinal questionnaire } & 103\end{array}$

Appendix C: Self-Report questionnaire - Experiment 1 and $4 \quad 109$

Appendix D: Statistics of Experiment $1 \quad 111$

Appendix E: Material by condition $\quad 118$

Appendix F: Self-Report questionnaire - Experiment 2 and $3 \quad 120$

Appendix G: Statistics of Experiment 2 122

$\begin{array}{lr}\text { Appendix H: Statistics of Experiment } 3 & 128\end{array}$

Appendix I: Statistics of Experiment 4 


\section{Index of Figures}

Figure 1. Attitude-change index towards the target issue as a function of message repetition and argument quality

Figure 2. TPR and $\mathrm{CO}$ values during the speech task as a function of message repetition $\quad 48$

Figure 3. Attitude-change index towards the target issue as a function of demands and message quality

Figure 4. Attitude-change index towards the target issue as a function of motivational state and argument strength for the presence condition

Figure 5. Attitude-change index towards the target issue as a function of message repetition and argument quality 


\section{Index of Tables}

Table 1. Proportion of favorable and unfavorable thoughts as a function of message repetition and message quality

Table 2. Proportion of favorable and unfavorable thoughts as a function of message repetition and message quality 


\section{Overview}

A previous encounter with a performance task is known to impact subsequent cognitive and motivational processes. However, theorists and researchers generally focus on one or the other of these effects. Here, we aim to interrelate these perspectives, specifically two well-grounded models: the Familiarity as a Regulation Mechanism Model and the Biopsicossocial Model of Challenge and Threat.

The Familiarity as a Regulation Mechanism Model (FARM model, Garcia-Marques, 1999; Garcia-Marques \& Mackie, 2007) is anchored on a large corpus of empirical evidence that shows that previously encountered information is processed analytically and in less detail. It assumes and empirically demonstrates that familiarity is a regulation mechanism responsible for decreasing the probability of analytic processing. The main rationale of this model is that when individuals reencounter a stimulus that was previously processed, it matches information stored in memory resulting in an implicit feeling of familiarity. Consequently, individuals learn to rely on familiarity as a signal that they can rely on an already available response in memory with no need to engage in deeper processing; that is, individuals can process the stimulus superficially rather than on its specificities (see Chapter $1)$.

Social psychophysiological theory has also focused on and demonstrated the impact of familiarity on motivational states. According to Blascovich' Biopsychosocial Model (2008), challenge and threat are activated as a function of whether or not individuals evaluate themselves as having sufficient or insufficient resources to meet or exceed situational demands (see Chapter 2). In this evaluation process, familiarity signals resources increasing the likelihood of challenge motivation. Specifically familiarity is based on previous successful learning and/or prior exposure to the stimulus or situation. Consequently, individuals evaluate the task as challenge, an effect typically marked by a specific pattern of cardiovascular responses. For example, cardiovascular responses consistent with challenge are exhibited when individuals perform in a motivated performance situation involving a well-learned task (Blascovich, Mendes, Hunter and Salomon, 1999) or when well-rehearsed attitudes are accessible to decision-making processes (Blascovich Blascovich, Ernst, Tomaka, Kelsey, Salomon \& Fazio 1993; Fazio, Blascovich \& Driscoll, 1992). 
These two lines of research suggest that the experience of familiarity is related to specific cognitive and motivational processes. Here, we purpose that both Blascovich's and Garcia-Marques's (1999) views are congruent and complementary. They overlap in one central aspect - they both argue that familiarity signals the cognitive system with an experience of resources available and complement each other by offering the motivational and cognitive implications of engaging in such experience. This overlap allows us to deduce several assumptions and test relevant hypotheses. Here we explore that deductive process and approach empirically some of these assumptions.

Our first approach was to integrate the cognitive and motivational impacts of familiarity and to study the relationship between them. Replicating Claypool et al.'s experiment (2005) on how familiarity induces non analytic processing, we aim to show that motivational state of challenge (measured by physiological activation indexes) accompanies such processing. We further address the relationship between these two effects, expecting the physiological correlates of the motivational state mediate familiarity effects on how persuasive information is processed.

Our second aim was to test if the motivational state of challenge generally (i.e., even if not elicited by familiarity) engages individuals in non-analytic processing. In the relevant experiment, challenge and threat were manipulated prior to the information-processing task (the same used in experiment 1) eliminating the familiarity path. If the motivational state of challenge impacts information-processing through an experience of resources availability, then, challenge should reduce analytic processing.

The relationship between the cognitive and motivational effects of familiarity on information-processing was further explored by manipulating task engagement. Both models assume task engagement as a modulator of how familiarity impacts cognitive processing or motivational processes. However they differ in how they describe such modulation. According to the FARM model, the impact of familiarity on processing is not dependent upon a high level of task engagement. On the contrary, the effect is more noticeable when engagement in moderate. For the BPS model task engagement is a necessary condition for engagement in challenge and threat motivation. As such, if our previous experiments show that both challenge and familiarity decrease analytic processing, it is expected that challenge only affects information-processing when task engagement is increased, whereas, it is expected that the familiarity effects are independent of such variable. We tested this hypothesis in the subsequent experiments (3 and 4). 
Section I 


\section{Chapter I: Familiarity, Cognitive Processing and Motivational States}

The impact of familiarity on information-processing has been a topic of growing interest in recent decades. In different subfields of social psychology, research has demonstrated that the familiarity of contexts affects various cognitive processes. For example, familiar situations can affect the way information is retrieved from memory (Jacoby, Woloshyn \& Kelley, 1989); the way other people are perceived (e.g. GarciaMarques \& Mackie, 2007); the perceptions of available resources to cope with stressful tasks (e.g. Blascovich, Ernst, Tomaka, Kelsey, Salomon \& Fazio 1993); liking towards abstract (ambiguous) stimuli (e.g. Zajonc, 1968; Zajonc, Shaven, Tavris, \& Van Kreveld, 1972); and even mood (e.g. Garcia-Marques, 1999; Garcia-Marques, Mackie, Claypool \& GarciaMarques, 2010).

This dissertation focuses on the impact of familiarity on cognitive processing and motivational states. Familiarity impacts a wide range of cognitive processes including perception, memory and impression-formation. Importantly here, familiarity impacts cognitive evaluation processes involved in the activation of motivational states of challenge and threat. In this thesis, possible links between cognitive processes and these motivational states were examined. The main purpose was to clarify whether the effects of familiarity on cognitive processing and motivational states involve independent parallel processes or whether they result from a single shared process.

\section{The impact of familiarity on cognitive processing.}

The demonstration that familiarity impacts cognitive processing has been well documented in different domains. For example, familiarity, increased via repetition, can bias judgments and the way information is retrieved from memory. One typical demonstration is provided by the well-known false fame paradigm (Jacoby, Woloshyn \& Kelley, 1989). In this study, participants read a list of non-famous names under a condition of divided attention (where they had to monitor an audio list of random numbers and signal any time they identify three consecutive odd numbers) or a condition of full attention (where they just reading the names). Then, participants read another list which now included famous names, repeated non-famous names (i.e. names previously shown in the first list) and new nonfamous names. Their task was to read each name and judge it as famous or non-famous. The results revealed that the repeated names (i.e. old non-famous names) were judged mistakenly 
as famous more than were the new ones (i.e. new non-famous names) in the divided attention condition. This means that, under certain circumstances like cognitive load, familiarity can impact cognitive processing directly.

Additionally, familiarity can also serve as an informative cue to one's decision processes. Evidence for this is found in all the experiments regarding the truth effect. These studies (e.g. Arkes, Hackett \& Boehm, 2006; Begg, Anas \& Farinacci, 1992) consistently find that repeated statements are judged as more truthful than unrepeated ones. This has been interpreted as a sign that individuals use their subjective experience of familiarity with the stimulus to ground their decisions (Begg \& Armour, 1991). Another example of how familiarity cues decision processes is provided by Halberstadt and Catty (2008). In their investigation, participants rated the popularity of a pair of tunes in terms of which was the most popular. One of each pair was a repeated tune and the other a new one. Half the participants were instructed to think about the reasons why they thought the tune they chose was the most popular (reasoning condition). The other half (control condition) did not receive this instruction. The results demonstrated that those in the control condition were more likely than "reasoners" to choose in accordance with their subjective reactions, i.e., their own familiarity with the stimuli. "Reasoners", on the other hand, chose according to other valid information besides their subjective experience.

These examples demonstrate how the experience of familiarity has a direct impact on cognitive processes by serving as a cue to drive information-processing. However, other evidence suggests that familiarity does not only have a direct impact on cognitive processing via these cognitive biases, but it also seems to affect how deep information is processed. Researchers in the cognitive and social cognitive literature have argued that when one reencounters a person or an object, he or she likely experience fluency or ease in processing its details (Schwarz \& Clore, 1996; Winkielman \& Cacioppo, 2001). Specifically, some have argued that this fluency is experienced as a feeling of familiarity (e.g. Garcia-Marques, 1999; Jacoby \& Kelley, 1990; Schwarz, 2004; Whittlesea, 1993) and such an experience is related to depth of information-processing. Garcia-Marques (1999, 2003, 2009) and her colleagues (Garcia-Marques \& Mackie, 2001; 2007; Garcia-Marques, Mackie, Claypool \& GarciaMarques, 2010) grasped this idea and explicitly posited that such feeling of familiarity may signal the need/or lack of need of deeper processing of information. Indeed, evidence within the FARM has demonstrated strongly that repetition can modulate the activation of the "modes of processing". 
Modes of processing and the regulatory role of feeling of familiarity. The assumption that familiarity regulates information-processing modes implies that information can be processed in different ways. The notion of "modes of processing" is associated with the widespread dualistic view that information can be processed in two qualitatively different modes: one based on the activation of prior knowledge (referred to as non-analytic processing below) and another one based on the computation of a new response (referred to as analytic processing below). Despite some conceptual differences that go beyond the purpose of this thesis (for appropriate reviews see, Chaiken \& Trope, 1999; Evans, 2008; Smith \& Decoster, 2000; Strack \& Deutsch, 2004), non-analytic processing is characterized by the activation of top-down processes that require little processing resources and motivation, whereas analytic processing is characterized by the activation of bottom-up processes that require resources and motivation.

Because top-down processes are driven by superordinate knowledge structures in memory, like schema or stereotypes (e.g. Higgins, King \& Mavin, 1982; Bodenhausen, Kramer, \& Siisser, 1994) non-analytic processing is more associated with superficial treatment of information. Evidence of non-analytic processing is found, for example, in the greater impact of category-based information on impression-formation (Brewer, 1988; Fiske \& Neuberg, 1999); in a greater impact of stereotypic information on judgments (Devine, 1989; Payne, 2008); and in an equal impact of strong and weak arguments in attitude-change (Chaiken, 1980; Petty \& Cacioppo, 1999; Wagner \& Petty, 2011).

On the other hand, because bottom-up processes are guided by new stimulus input rather than by pre-existent knowledge structures, analytic processing is associated with the detailed processing of the information as demonstrated in research revealing a greater use of individuating information when forming impressions about a target (Brewer, 1988; Fiske, Lin \& Neuberg, 1999); in the control of the impact of stereotypical information on judgments (Devine, 1989; Payne, 2008); or in a greater persuasive impact of strong over weak arguments (Chaiken, 1980; Petty \& Cacioppo, 1999; Wagner \& Petty, 2011).

Researchers such as Higgins (1989) and colleagues (Higgins \& Brendel, 1995), have suggested that the activation of a bottom-up type of processing is determined by the adequacy of the match between the input and its mnesic representation. Assuming that the information stored in memory varies in terms of frequency and recency of activation and that activation declines over time as a negative function of the frequency of its use, the investigators argue that if information is activated before the stimulus presentation and if it is 
applicable to it, then, the information-processing is likely to be guided by these principles of associative information activation.

A similar argument is made by Johnston and Hawley (1994). According to these authors, cognitive resources are managed in order to be applied only to relevant situations. Thus, the cognitive system does not waste valuable attentional resources deeply processing expectancy-consistent or familiar material that can be encoded quickly and efficiently by simple and associative processes. As expected, when information matches existing templates in memory, individuals will only extract the gist of the material. However, in the presence of novel or unfamiliar stimulus, individuals cannot rely on memory templates or other previous existing knowledge and have to engage in active searches in order to generate a novel response (Norman \& Shallice, 1986).

The integration of such evidence led Garcia-Marques (1999, 2003, 2009) to hypothesize that the match between the characteristics of the situation/stimulus and the relevant correspondent information in memory elicits a feeling of familiarity that signals the cognitive system to process information on the basis of what is accessible/known. Her rationale is as follows. If the information represented in memory matches the information currently received, that representation would be highly activated and accessible (GarciaMarques, 2003). As a consequence, processing becomes more fluent because individuals process information on the basis of what they already know rather than on the idiosyncrasies of the situation/stimulus. As such, what regulates the activation of a mode of processing over the other is a feeling that signals the match of the received information to the one stored in memory (Garcia-Marques \& Mackie, 2001). If a match is detected, top-down processes are activated and subsequent processing is based on prior knowledge. Hence, familiarity regulates modes of processing by decreasing the probability of analytic processing.

These postulates are grounded in the aforementioned research of Higgins (1991) and Johnston and Hawley (1994) but also in other domains demonstrating that repetition decreases analytic reasoning. For example, Reder and Ritter (1992) ran an experiment on problem-solving. In it, participants were presented a series of arithmetical problems (i.e. multiplications, additions) that were repeated over time. For each problem, participants had to decide if they were able to give the correct answer or not within an interval of $850 \mathrm{~ms}$. The problems were selected such that the answers could not be calculated within that time interval (e.g. 64 x 78). If participants answered affirmatively, they had to decide if they wanted to retrieve or calculate the answer. The results showed that familiarity with 
components of the problem (e.g. similar multiplication coefficients) increased the preference for the retrieval option that in turn strongly predicted participants' feeling of knowing the answer (most notably in the case of novel problems that were similar to previously seen problems). In line with the FARM model, when individuals felt they knew the answer to a problem because some features of the problems were repeated, their judgments reflected a greater preference for the retrieval of a previously computed and available response from memory instead of the computation of a new one.

Recent findings congruent with FARM assumptions also suggest that a feeling associated with processing may regulate the activation of processing modes. Thompson, Turner and Pennycook (2011) demonstrated that the regulation of information-processing modes is driven by a "feeling of rightness" that signals the cognitive system that nonanalytic processing is adequate. In a series of studies, the authors manipulated the fluency with which information was processed and observed a strong relationship between the feeling of rightness related with their answers and decreased analytic processing. Even though the subjective feeling conceptually differs from the one postulated by GarciaMarques et al. (2001), Thompson et al.'s findings offer support for the FARM model in the sense that it shows that a feeling associated with processing ease is on the basis of a mechanism that regulates information-processing modes.

Direct evidence for familiarity as a regulation mechanism has been validated widely. In an early attempt to demonstrate the existence of this mechanism, Garcia-Marques and Mackie (2001) tested the impact of argument repetition on cognitive elaboration. Specifically, participants read a persuasive message containing either strong or weak arguments in favor of the effectiveness of weight loss centers. Additionally, half of the participants listened, via headphones, to an unrelated message that purportedly was only required in order to simulate an outside environment and, therefore, should not be their focus. After reading the message, participants expressed their opinions on the topic. Next, participants read a second persuasion message embedded with strong or weak arguments favoring the imposition of governmental controls on industries to minimize the effects of acid rain. For half participants (the ones who read the message wearing headphones), this was actually the same message that was played over the headphones. For the other half, the message was new. After reading the message, participants rated their opinion of the topic. Because the repetition of the message was assumed to promote a feeling of familiarity (Begg \& Armour, 1991; Jacoby, 1991; Schunn, Reder, Nhouyvanisvong, Richards \& Stroffolino, 
1997; Whittlesea, 1993), the authors hypothesized that in the repetition condition the argument-strength would not have a differential impact on attitude-change. The results showed that, as predicted, message repetition resulted in an equal persuasive impact of strong and weak arguments in attitudes, suggesting less analytic processing in this condition.

The same pattern of results was obtained by Claypool et al. (2004) using a similar paradigm with the exception that in the first phase all participants listened to an audio message (that was subsequently repeated or not) while reading the message. Again, those experiencing the second (duplicate) message as familiar (through previous auditory exposure) did not process its content as confirmed by the fact that they were equally persuaded by strong and weak arguments. Additional regression analysis demonstrated that perceived familiarity predicted non-analytic processing (the more perceived familiar, the less analytic processing).

These findings are extended by evidence demonstrating that repetition of information can also decrease the impact of individuating evidence on stereotypical judgments (GarciaMarques \& Mackie, 2007). In this experiment, participants read individuating information about an alleged perpetrator that was either exculpatory or incriminating. Their task was to deliberate about the perpetrator's guilt. Results from two studies demonstrated that when individuals experienced a feeling of familiarity, either induced by subliminal repetition of a photo (experiment 1) or by repetition of relevant individuating information about the target (experiment 2), analytic processing was decreased as shown by the reduction in impact of individuating evidence on judgments and increased sensitivity to category-membership information in this condition.

Moreover, the hypothesis that familiarity regulates the activation of one informationprocessing mode over the other is directly shown in Moons, Mackie and Garcia-Marques experiment (2009). Participants were presented a series of strong and weak arguments and expressed their agreement towards each one of them. Some were repeated and some were not. Using the process dissociation procedure (Jacoby, 1991), a procedure that allows the independent estimation of controlled (more deliberative) and automatic (more associative) components of information-processing, it was verified that the arguments' repetition promoted a dissociation in the processing components, such as, repetition increased the use of more automatic components. In other words, participants agreed more with repeated arguments due to automatic influences of familiarity (and independently of controlled processing). 
Taken together, the evidence reviewed here corroborates the FARM hypothesis demonstrating that repetition of information is somewhat associated with a greater use of processing strategies that privileges the use of prior knowledge that is accessible in memory.

Additional evidence supporting the FARM is also found in the literature that considers the impact of fluency on processing. Recall that for the FARM model and others (e.g. Garcia-Marques, 1999; Jacoby \& Kelley, 1990; Smith, 1994; Schwarz \& Clore, 1996; Winkielman \& Cacioppo, 2001), the match between the information received and that accessible in memory makes the processing more fluent. This fluency, experienced according to the FARM as a subjective feeling of familiarity, causes individuals to process information on the basis of what is already activated, decreasing the depth with which information will be subsequently processed. Therefore, the experience of fluency (associated with the feeling of familiarity or originated by other sources) should also promote decreases in analytic processes what has been, effectively, observed in different investigations. For example, Alter, Oppenheimer, Epley, Eyre and Rebecca's (2007) demonstrated in a series of studies that fluent situations reduce the impact of systematic cues on favorability ratings in persuasion. Participants were asked to read a persuasive ad regarding an mp3 song which was presented either with a headline in a font easy to read (fluency condition) or in a font difficult to read (disfluent condition). Moreover, the ad was embedded with strong or weak arguments favoring the quality of the $\mathrm{mp} 3$ and a photo of a prototypical expert in the subject (vs. non-expert) was presented. The results showed that when the headline's font was easy to read, participants neglected the quality of the arguments anchoring their favorability judgments on the heuristic cue presented (the photo of the expert), a strategy that, as we stated previously, is typically associated with non-analytic processing.

Furthermore, this impact of fluency on processing seems to occur even more subtly as Song and Schwarz's (2008) Moses illusion experiment demonstrates. In this study, participants were simply asked about how many animals of each kind Moses took on his ark when the flood took place. Half participants read this sentence in a fluent condition (i.e. font easy to read) and the other half read it in a disfluent condition (font difficult to read). The results demonstrated that the error embedded in this sentence (it was not Moses, but Noah) was not detected in the fluency condition, but it was in the disfluent one. This data suggests that in the condition where processing became fluent, participants engaged in a non-analytic mode of processing which made them disregard the details of the question. 
Even though these findings do not necessarily assume that fluency is experienced as a feeling of familiarity (for appropriate debates in the literature see for example Alter \& Oppenheimer, 2009), they do not necessarily conflict with the FARM predictions. In fact, the involvement of fluency in the experience of a feeling of familiarity has been widely reported in several investigations (e.g. Dewhurst \& Hitch, 1997; Whittlesea, 1993; Whittlesea \& Leboe, 2003; Whittlesea, \& Williams, 2000), as well as their interconnection. Evidence of the familiarity-fluency link has been documented in the findings that prior exposure to stimuli enhances the ease with which they can be processed (i.e., Winkielman, Halberstadt, Fazendeiro \& Catty, 2006) and, conversely, increasing the ease with which a stimulus is processed (e.g., by enhancing its clarity) can produce the perception that the stimulus has been previously seen (e.g., Whittlesea, Jacoby, \& Girard, 1990). Because fluency and familiarity are linked, authors (e.g. Schwartz, 2004) have suggested that any variable that facilitates processing leads to increased perceptions of familiarity, even when the facilitation derives from irrelevant characteristics like high figure-ground contrast, long exposure times, or preceding visual or semantic primes. As such, both fluency and familiarity seem to be part of a same mechanism that is responsible for decreasing the probability of analytic processing these studies converge on the conclusion that fluency of processing feeds into judgments, "presumably by making the presented information "feel" more familiar" (Schwarz, 2004, p. 6). However, this is still an empirical question that the literature has not addressed yet.

Summary.

The findings described above demonstrate that familiarity, even by subtle repetition of information, is sufficient to reduce the probability of analytic processing. This effect has been attributed to a subjective feeling that signals the cognitive system to rely on previous knowledge that is accessible and available in memory.

\section{The impact of familiarity on motivational states of challenge and threat.}

Familiarity has also been shown to impact the cognitive evaluation processes involved in the activation of motivational states of challenge and threat. In the tradition of cognitive appraisal theory (Lazarus \& Folman, 1984; Lazarus, 1991), these evaluations are activated in potentially stressful or threatening situations in which individuals evaluate (sometimes consciously but more often unconsciously) whether their personal resources 
meet the demands of the situation (Blascovich \& Tomaka, 1996; Blascovich, 2008). Blascovich and colleagues have demonstrated that individuals experience a motivational state of challenge when their evaluations of resources meets or somewhat exceeds the situational demands; but experience a motivational state of threat if they do not (e.g., Blascovich, Mendes, Tomaka, Salomon \& Seery, 2003; Blascovich, Seery, Mugridge, Norris \& Weisbuch, 2004; Mendes, Major, McCoy \& Blascovich, 2008; Tomaka, Blascovich, Kelsey \& Leitten, 1993; Tomaka, Blascovich, Kibler \& Ernst, 1997).

Contributing to this resource and demand evaluation process are several intraindividual (e.g. psychological or physical danger; abilities, skills, knowledge; dispositions; uncertainty, novelty and familiarity; required effort etc.) and inter-individual (e.g. social support) factors with familiarity being the one of our interest here. It has been postulated and empirically demonstrated that familiarity impacts perceptions of available resources which facilitates meeting situational demands (Fazio, Blascovich \& Driscoll, 1992; Blascovich et al., 1993; 1999). By doing so, familiar situations are typically associated with task engagement and challenge.

The dualistic activation of motivational Challenge and Threat. How cognitive appraisals or evaluations influence behavior has been a topic of much research in social psychology, especially in the stress literature (Blascovich \& Katkin, 1993; Cox, 1978; Lazarus, 1991; Lazarus \& Folkman 1984; Obrist, Gaebelein, Teller, Langer, Grignolo \& Light, 1978; Tomaka, \& Blascovich, 1994; Tomaka, \& Palacios-Esquivel, 1997; Taylor, Seeman, Eisenberger, Kozanian, Moore \& Moons, 2010; Tomaka, Palacios, Schneider, Colotla, Concha \& Herrald, 1999). Research in this domain demonstrates that cognitive and emotional processes are dependent on evaluations that individuals make with regard to the significance and outcome of a specific encounter.

Early on, Lazarus and colleagues (Lazarus 1966, 1991; Lazarus \& Folkman 1984) posited and validated that, in the face of encountering a situation that may be helpful or detrimental for one's well-being, individuals appraise whether their personal resources are enough to meet the situational demands. The outcome of this appraisal results a general state that defines the subsequent biological, emotional, behavioral and cognitive reactions, such as, a threat state is elicited if individuals anticipate that harm may be imminent and a challenge state results if the person feels confident about mastering the task (Lazarus, 1991). 
Following on Lazarus' work, but eschewing the label "appraisal" because of its strong implication of a conscious process, Blascovich and his colleagues developed the Biopsychosocial Model of Challenge and Threat (BPS) that explores the implications of cognitive "evaluations" more deeply, and their interactions with biological, emotional and behavioral responses in the activation of motivational states. Congruent with Lazarus's conceptualization, Blascovich and colleagues (Blascovich \& Mendes, 2000; Blascovich \& Seery, 2006) have defined challenge as a motivational state activated when the evaluation of available resources is sufficient or exceeds the perceptions of demands necessary to deal with the situation. Challenge is described by the BPS as an approach-approach type of motivation generally with positive affective tone (Blascovich \& Mendes, 2000). Conversely, a motivational state of threat is activated when individuals evaluate fewer resources than the ones needed to deal with the situation. It is described as an approach-avoidance type of motivation typically with a less positive or even sometimes negative affective tone.

According to the now well-established BPS model (Blascovich, et al., 1999, 2003, 2004; Mendes et al., 2008; Tomaka \& Blascovich, 1994; Tomaka, et al, 1993, 1997; for a review see Blascovich, 2008; Blascovich \& Seery, 2006), challenge or threat occur in potentially threatening (and therefore stressful) motivated performance situations (i.e., ones that require instrumental cognitive responses) that are self-relevant and, hence, goal-relevant to the individual and, consequently, task engaging. Evaluation of resources and demands moderate the activation of the two oppositional motivational states: challenge and threat. Hence, such situations require active rather than passive coping (e.g. giving a speech vs. watching a video; Blascovich \& Mendes, 2000; Tomaka et al., 1993).

Within motivated performance situations, task-engaged individuals typically exhibit significant increases in heart rate (i.e. how fast the heart beats per minute) and ventricular contractility (i.e. contractions of the left ventricle of the heart) from resting baseline levels (i.e. within the goal-relevant situation; Obrist, 1981; Blascovich, Mendes \& Seery, 2002). Once individuals are task-engaged, challenge and threat are modulated by the resource and demand evaluation process (Blascovich, 2008). If task engagement does not occur, then challenge and threat are not differentiated physiologically (as demonstrated by Tomaka et al. 1993, experiment 3).

The engagement in the motivational state of challenge or threat, as a function of a resources and demands evaluation ratio, is typically indexed by the activation of a specific pattern of cardiovascular (CV) responses. Tomaka et al (1997) first elucidated these findings 
by demonstrating that physiological responses could be aggregated within two specific motivational patterns (i.e., challenge and threat) based on similar ones (physiological toughness and weakness) as proposed by Dienstbier'(1989) theory of physiological toughness in his work on rodents.

Dienstbier's (1989) observed that animals exhibited two different patterns of cardiovascular responses when performing in potentially life threatening situations (e.g. avoiding a predator): physiological toughness and physiological weakness. The physiological toughness pattern is characterized by stimulation via the sympathetic neural and adrenal medullary (SAM) axis. As a consequence, myocardial (i.e., heart muscle) contractility, more specifically left ventricle contractility (VC) and heart rate (HR) increase via increased sympathetic stimulation of the myocardium. Further, according to Dienstbier, the adrenal medullary release of epinephrine into the bloodstream not only further increases HR but dilates the arteries, thereby decreasing total systemic peripheral vascular resistance (TPR) and, in combination with increased heart rate and myocardial contractility, increase blood flow as indexed by cardiac output (CO). The physiological weakness state, on the other hand, also includes activation of the SAM axis, but accompanied by the activation of the hypothalamic pituitary adrenal (HPA) axis. The latter results in the release of cortisol into the blood stream. This obstructs the SAM-driven decreases in TPR and the SAM-driven increases in CO. In turn, the HPA axis activation usually results in little change or even increases in TPR and decreases or no changes in CO but an increase in mean arterial blood pressure.

Testing Dienstbier's (1989) conceptualization in humans, Tomaka et al. (1993) posited that, under conditions of potential threat in goal relevant situations, these patterns could also be activated via the results of situational resource/demand evaluations in humans. During challenge (more resources than demands), individuals exhibit a pattern of physiological toughness, whereas in situations of threat (more demands than resources), individuals exhibit a pattern similar to physiological weakness. However, this study was based on correlations between pre-task demand/resource evaluations and resulting cardiovascular responses during task performance.

To test their theoretical notions experimentally, Tomaka et. al (1997, experiment 1 ) manipulated challenge and threat evaluations prior to task performance and recorded the $\mathrm{CV}$ responses during performance of a mental arithmetic task. Challenge and threat evaluations were manipulated via the type of instructions furnished. In the threat condition, participants 
were instructed that they should perform as quickly and as accurately as possible because their performance was going to be scored for speed and accuracy. In the challenge condition, participants were told that they should think of the task as a challenge and they should think of themselves as capable of meeting that challenge. In this condition, no instruction regarding performance evaluation was given. After the instructions and prior to beginning the task, evaluations were assessed by asking participants how threatening they thought the mental arithmetic task would be (assessing perception of demands); and how able they thought they were to cope with it (assessing perception of resources). Consistent with cognitive evaluations of challenge, the results showed that those who received the challenge instructions perceived more resources than the ones who receive the threatening ones. Importantly, those who appraised the situations as challenging exhibited the pattern of responses consistent with Dienstbier' physiological toughness pattern (i.e. significant increases in $\mathrm{HR}, \mathrm{VC}$, and $\mathrm{CO}$ and decreases in TPR) and those who appraised the situation as threatening exhibited a pattern of responses similar to physiological toughness (i.e. significant increases in $\mathrm{HR}$ and $\mathrm{VC}$ but decreases or no changes in $\mathrm{CO}$ and increases in TPR).

The causal relationship between the cognitive evaluations and the cardiovascular responses is rather unilateral (i.e., cognitive evaluations lead to challenge or threat) as shown by Tomaka et al's (1997, experiment $2 \& 3$ ) investigation. The authors induced cardiovascular patterns that mimicked the cardiovascular patterns consistent with challenge and threat and then observed their impact on cognitive evaluations to test whether the manipulated cardiovascular responses could predict the cognitive evaluations. In experiment 2, participants either pedaled a static bicycle at a moderate workload (50 watts), an activity that mimics physiological challenge; or sat stationary on it, which served as control condition. In experiment 3, participants either immersed their hands in a warm or ice bath. The warm bath mimicked physiological challenge patterns, whereas, the cold pressor mimicked physiological threat. In each study, participants reported demands and resources for an upcoming serial subtraction task during the manipulation (i.e., while on the bicycle or with hand immersed in the bath). The results in both studies demonstrated that the cognitive evaluations of resources and demands did not vary as a function of the physical manipulations. This suggests that the experience of the different cardiovascular patterns does not differentiate demand/resource evaluations, ruling out the hypothesis of reversed causality. 
Research has consistently validated the moderation role of the cognitive evaluations in the activation of the $\mathrm{CV}$ challenge and threat patterns. For example, research within the BPS model has shown that inducing threat via having participants engaging in a collaboration task with a stigmatized other (Blascovich et. al, 2001) induces the cardiovascular pattern of threat. Similarly, inducing threat by putting participants in interaction with a member form a minority group (e.g. Blacks) also increases a cardiovascular pattern of threat (Mendes, Blascovich, Lickel \& Hunter, 2002, see also, Mendes et al., 2001). Further evidence also suggests that violating stereotype-expectancies (e.g. Asian students speaking with accents) (Mendes, Blascovich, Hunter, Lickel \& Jost, 2007); and increasing sexism salience in women is associated to cardiovascular threat (Eliezer, Major, \& Mendes, 2010; for more examples see Blascovich, 2008; Blascovich, Mendes, \& Seery, 2002; Blascovich \& Seery, 2006).

The impact of these motivational states on performance has been widely documented within and outside the BPS model. Typically, challenge has been associated with better performance and threat to poorer performance. For example, compared to a threat state, challenge is usually associated with better athletic performances (Blascovich, et al., 2004); increased speed in pairwise preferences decisions (Blascovich et al., 1993; Fazio et al., 1992); increased accuracy for serial subtraction (e.g. Tomaka \& Blascovich, 1994; Vick, Seery, Blascovich \& Weisbuch, 2008) or object and pattern recognition judgments (Blascovich et al., 1999); and better performances on the Remote Association Task (Seery, Blascovich, Weisbuch \& Vick, 2004) (for reviews see Blascovich, 2008).

Also outside the BPS model, there is evidence that evaluation instructions lead to differences in performance outcomes, Drach-Zahavy and Erez (2002) varied the instructional set to correspond to either challenge or threat evaluations and found that the evaluation instructions led to differences in performance outcomes, such that challenge instructions increased accurate predictions of the stock value of a series of companies. Likewise, O'Connor, Arnold, and Maurizio (2010) showed, that when individuals are primed with challenge evaluations, via subtle instructions informing that the task is easy to accomplish (vs. subtle instructions informing that the task is a difficult one), they performed a negotiation task shown resulting in more favorable agreements reached while negotiating a school project.

Such an improvement effect of challenge on performance has led Wright and Kirby (2003) to question whether this effect is due to the engagement in a specific motivational 
state, but rather due to a greater effort exerted in these situations. Drawing from research on the psychophysiology of effort (Brehm \& Self, 1989; Light, 1981; Obrist, 1981; Wright \& Kirby, 2001), Wright and Kirby (2003) suggested that the amount of effort one puts in a task is associated, per se, with increases in sympathetic activation, producing similar CV effects to those proposed to be associated with challenge, namely increases in cardiac activity indexes (i.e. myocardial contractility, heart rate, and systolic blood pressure). Based on this, they argue that it is possible that challenged participants tend to exhibit greater cardiac activity than threatened participants simply because they exert more effort. If, in turn, challenged participants exert more task effort, then the better performances associated with this state, can be due to the greater effort exerted and not necessarily because of the engagement in a specific motivational state.

To make their point clear, Wright and Kirby (2003) presented an effort-based alternative interpretation using the Tomaka et al.'s (1993) study where challenge improvement effects on performance were found. In this study, participants were asked to perform some moderately difficult mental subtractions. They were asked either to do it silently (challenge condition) or verbally, i.e., out loud (threat condition). Results demonstrated that those who had to perform the task silently exhibited greater increases in cardiovascular reactivity (consistent with challenge) and gave more correct answers, than did those who performed the task out loud, who exhibit less cardiac activity and poorer performances. A BPS interpretation of this data, suggests that the silent performance may have decreased perceptions of required effort, which in turn, decreased the demands-toresources ratio, resulting ultimately in challenge. Conversely, the "public" performance may have increased required effort which in turn, increased the demands-to-resources ratio leading to threat. But, if according to Wright and Kirby's (2003) point of view, those in the "public" condition made higher demand-to-resources ratios (as the BPS model predicts) and, as a consequence, predicted a negative outcome (e.g. public humiliation), they may have had lower cardiac activity and poorer performances, not necessarily because they engaged in a specific motivational state, but because they "may have been more inclined to despair and abandon their effort to succeed" ( $p$. 231). In other words, they may have exhibited lower cardiac activity and poorer performances simply because they exerted less effort and disengaged from a task for which they predicted failure.

Even though this is an interesting interpretation, for which no direct empirical evidence exists so far, Wright and Kirby's (2003) argument is ruled out by all the evidence 
that shows that even when effort is controlled across experimental groups, differences in $\mathrm{CV}$ responses attributed to challenge and threat states emerge. Take for example the experiments involving the deliverance of a speech (e.g. Blascovich et al., 2001, 2004; Eliezer et al., 2010; Mendes et al., 2002; Weisbuch-Remington et al., 2005). In these paradigms, all individuals, randomly assigned to a threat or challenge condition, are asked to deliver a speech. As Blascovich et al. (2003) points out, delivering a speech has metabolic requirements associated to it which are not expected to differ across groups because speaking was never more difficult or required more physiological effort for one group and not for the other. What varies in these experiments is the context in which the speech takes place. Consistent with a challenge and threat evaluation predictions, $\mathrm{CV}$ responses are greater when participants give a speech to an in-group member, in comparison with an out-group member (e.g. Mendes et al., 2002) or speak about a pleasant topic instead of their own death (e.g. Weisbuch-Remington et al., 2005). Thus, maintaining constant the metabolic activity of giving a speech, it seems hard to understand how effort by itself is sufficient to explain differences verified across the experimental conditions.

This effort-based interpretation is also ruled out by the studies demonstrating that challenge does not always lead to better performance. Take for example, Hunter's investigation (2001). In this study, participants were informed that they would either have to read (challenge condition) or sing (threat condition) the U.S. national anthem after performing a criterion task. Before they engaged in the criterion task, participants either read or sang the first two stanza of the anthem to induce the correspondent state. Immediately afterwards, participants performed the criterion task that consisted of watching pairs of words and read some of them aloud. By the end of the presentation, participants were instructed to recall all the words they saw and identify intrusions among a list of words. Results revealed that those who were threatened by the possibility of signing the national anthem, exhibited greater decreases in cardiac activity and recalled more words correctly and indentified more intrusions correctly, than those who were challenged. If the greater CV reactivity was merely tapping greater effort as Wright and Kirby suggest (2001, 2003), then we should not expect these effects. We should expect that individuals from whom CV was higher to always perform better, which does not seem to be the case.

Similarly, Feinberg and Aiello (2009, experiment 1) and Alter, Aronson, Darley, Rodriguez and Ruble (2010) have found that challenge leads to poorer performance than threat. Alter et al. (2010), for example, observed that when a stereotype is made salient (e.g. 
by making black students aware of their minority group membership) and individuals became threatened, the typical stereotype-threat impaired-performance effect (for reviews see, Aronson \& McGlone, 2009; Schmader, Johns, \& Forbes, 2008) is eliminated if the task is framed as challenge, i.e., if participants are instructed to think about the task as a great opportunity to grow and learn something new and helpful. Even though no CV data were furnished in this study, threatened participants had better performances. If effort is translated in better performances and if, according to Wright and Kirby's rationale threatened participants tend to exert less effort on performance, then we should expect these individuals to always perform worse which, again, does not seem to be the case.

Familiarity and Challenge. Several factors directly influence the resources and demands evaluations responsible for challenge and threat activation. As stated previously, one assumption of the BPS model is that familiarity brought by previous knowledge is associated with the activation of a motivational state of challenge.

Factors like skills, abilities, social support and/or previous knowledge have been shown to influence resources evaluations (for review see, Blascovich, 2008; Blascovich \& Seery, 2006). For example, if an individual has good skills in spoken-French it is likely that he perceives himself has having sufficient resources to engage in a conversation in French. Factors like danger, uncertainty, risk, required effort, dispositions and/or social evaluation have been shown to influence demands evaluations. For example, an individual with good skills in spoken-French can perceive greater situational demands if he is aware that the conversation will be evaluated by a French native.

Some authors (e.g., Feinberg \& Aiello, 2009; Maier, Waldstein, \& Synowski, 2003; Wright \& Kirby, 2003) have challenged this broad conceptualization of resources and demands stating that it is difficult to understand how an individual can accurately appraise the resources and demands required when multiple factors are involved. As a consequence, these same authors have questioned the cognitive ratio as a valid approach to challenge and threat.

For the BPS model it is not so much the question of the exact levels of resources and demands that lead to challenge or threat, but rather the subjective experience of evaluating more, or less sufficient resources to face the expected demands of the task. Blascovich et al. (2003) defends that individuals are not cold calculators of the exact resources and demands because they are cognitively and affectively influenced by what is happening in the context 
surrounding them and this influence can generally occurs below the levels of awareness. The studies that found challenge and threat effects using subliminal manipulations clearly demonstrate this (Mendes, Weisbuch, Seery \& Blascovich, 2002; Weisbuch-Remington et al., 2005). Weisbuch-Remington, et al. (2005, experiment 1), for example, found that participants exposed subliminally to negative religious symbols prior to deliver a speech about their own death exhibited a $\mathrm{CV}$ pattern consistent with threat, than participants exposed subliminally to positive symbols who were more challenged.

The influence of each factor on resource and demand evaluations is not independent (Blascovich \& Mendes, 2000). In fact, one factor can simultaneously influence the evaluation of resources and the evaluation of demands. For example, abilities can simultaneously increase evaluations of resources, based on the available skills to perform, as well as, decrease demands because it requires little effort. As such, the impact of each factor over the resource and demands evaluations is said to overlap, be synergistic and/or interact to some extent, making this process a complex one and occurring deliberatively or automatically (Blascovich, 2008).

In this interactive and iterative process, familiarity is a relevant factor that can directly disrupt both of resource and demand evaluations. According to Blascovich et al. (1993), familiarity can both increase one's personal resources, based on the previous knowledge it provides, and decrease situational demands, based on little required effort. As such, when the situation is familiar, individuals will likely evaluate themselves has having enough or more resources to cope with the situation based on the previous knowledge they have stored. Conversely, when the situation is unfamiliar or novel and no previous knowledge is available, individuals tend to evaluate resources as less than the ones required. Because in familiar situations, resources tend to overcome demands, familiarity is typically associated with challenge, whereas novelty is more associated with threat.

This argument has been validated in a series of investigations (Fazio et al., 1992; Blascovich et al., 1993, 1999; Kelsey' Blascovich, Leiten, Schneider, Tomaka \& Wiens' 2000) that demonstrate that task-practice (through repetition) increases the CV pattern of responses associated with a challenge. For example, in Blascovich et al. experiment (1993), participants were asked, for an initial task, to observe a set of 30 abstract paintings. Half of the participants were instructed to decide whether they liked or disliked each painting by saying aloud "like", "like strongly", "dislike", or "dislike strongly." The other half (those assigned to the novel condition) were instructed to say aloud the predominant color of each 
painting and the percentage of that color contained within the painting. On the subsequent task, all participants were informed that they would be shown several pairs of paintings and their goal was to indicate which painting in each pair they most preferred. For half the participants (those in the familiar condition), the paintings presented were those that they have seen previously and for which they have already formed an opinion. For participants in the novel condition, the paintings were completely new. As expected, results showed that participants in the familiar condition were faster in making their judgments and exhibited the cardiovascular pattern of responses consistent with a challenge state. Conversely, participants exposed to novel unrehearsed condition were slower in their judgments and exhibited a cardiovascular pattern of responses consistent with threat.

Consistent with this findings, Kelsey et al. (2000) and Blascovich and colleagues' (1999) experiments in addition to showing that familiarity impacts individuals' motivational states they also show that the impact of familiarity on challenge and threat is moderated by the presence of others. In Blascovich et al. (1999) experiment, participants learned a specific task (either a number-categorization tasks or a pattern-recognition task) to a predetermined criterion. Then, they were randomly assigned to perform the same task (familiar condition) or the other one (novel condition) in one of two social conditions: either alone or in the presence of two observers, introduced as two students who were going to observe participants' performance. Congruent with previous findings, the results indicated that, compared to those performing in the novel situation, those in the familiar condition performed better and exhibited a cardiovascular pattern of responses consistent with challenge. However, this effect was only valid in the presence of the observers because only those in the presence condition were task-engaged (as shown by significant increases in HR and VC from baseline values occurring only for this group). Remember that because task engagement is a necessary condition to assess challenge and threat, these motivational states were not differentiated for the alone group. These findings, thus, demonstrate that the presence of others is an important moderator of the effects of familiarity on challenge and threat.

\section{Summary.}

The findings reviewed here clearly demonstrate that, under task-engaging situations, two qualitatively different motivational states are activated as a function of a cognitive evaluation-based ratio of resources and demands. Task familiarity provides resources to the 
individual which generally activates a state of challenge, an effect that seems to be moderated by the presence of others. The presence of others during a performance of a welllearned task likely increases task engagement and increases the probability of positive social consequences of good performance whereas the presence of others during a performance of a novel also increases task engagement but increases the probability of negative social consequences of poor performance. Indeed, in a gain/loss framing study involving challenge and threat, Seery, Weisbuch \& Blascovich (2009) demonstrated that gain and loss framing of a task leads to challenge and threat cardiovascular responses, respectively.

In this chapter, we have reviewed how familiarity impact cognitive processing, focusing on how it moderates cognitive processing style (an assumption from the FARM model) and how it moderates motivational states (an assumption from the BPS model). In the next chapter we explore how the two are related, building a bridge between the two underlying models. 


\section{Chapter II: Hypothesizing the relationship between the FARM and the BPS model}

In the previous chapter, the impact that familiarity appears to have on cognitive and motivational processes was reviewed. Evidence from the FARM model (Garcia-Marques, 1999; Garcia-Marques \& Mackie, 2007) has shown that familiarity has a regulatory role on the activation of information-processing modes. Evidence from the BPS model (Blascovich, 2008; Blascovich \& Tomaka, 1996; Mendes et al., 2007) suggests that familiarity impacts the cognitive evaluation processes that underlie the activation of superordinate motivational states. Here, how these two effects may be related and the bridge that can be built between the two underlying models is discussed.

Briefly, the rationale here is based on the notion that the two models bridge the relevance and definition of the experience of available resources. Familiarity is assumed by the FARM as a sign of the presence of a category of resources to deal with a task. Challenge motivation is assumed by the BPS model to be associated with the evaluation of available resources to deal with task demands in a motivated performance situation. Thus, familiarity may be evaluated as a resource, and all other things being equal, lead to the motivational state of challenge. This hypothesis and its implications are explored in this chapter.

\section{Familiarity and Challenge.}

Familiarity is assumed by the FARM model to develop as a consequence of top down processes facilitation resulting from a match between the characteristics of the situation/stimulus and the relevant correspondent information in memory. This match in memory is associated with more fluent processing (Garcia-Marques, 2009). Because this association signals the availability of a resource, it marks the situation as one with which one is more likely to cope (at least as well as in the past) with all other evaluations being equal. According to the BPS model, such familiarity increases the ratio of evaluated resources relative to demands.

As reviewed in Chapter 1, challenge is a motivational state that is marked by the stimulation of the sympathetic neural and adrenal medullary (SAM) axis resulting in a specific "challenge" pattern of cardiovascular responses: increased heart rate (HR) and ventricular contractility (VC) accompanied by decreases in systemic peripheral vascular resistance (TPR) and increases in cardiac output (CO); i.e., blood flow. 
As Blascovich et al. (2003) suggest, although motivational states may sometimes be validly indexed via self-report, they are more objectively indexed via concomitant physiological measures. Furthermore, any process, such as one involving increasing familiarity with a task that increases the ratio of task performance situational resources to demands should result in more of a challenge motivational state as indexed by the challenge cardiovascular pattern. Recall that Blascovich et al.'s $(1993 ; 1999)$ data already demonstrate that familiarity with an upcoming task, through previous practice, increases the activation of a motivational state of challenge. What is not known, however, is whether there are other factors linking familiarity to increased resources. Additionally, it is not known if activation of familiarity within motivated performance situations impacts information processing as it has been shown by the FARM model.

Several aspects of both models can be considered in these arguments. The first is that in the FARM model familiarity is assumed to be independent of the degree of individuals' task engagement. According to the BPS, however, challenge requires task engagement.

Second, whereas familiarity is assumed to be automatically activated as result of individuals attending to a stimulus/task, challenge results from a ratio of two different components: evaluated resources and evaluated demands, which can occur deliberately (i.e., consciously), automatically (i.e., unconsciously), or both (Blascovich, 2008). This evaluation process is highly complex because multiple factors enter into the resource/demand evaluations (e.g. psychological and physical danger/safety, uncertainty/certainty, novelty/familiarity, required effort, skills and knowledge, the presence of others, affective cues, attitudes and beliefs, etc.). As such, the resources/demands ratio is only partially influenced by familiarity. Furthermore, these factors are not completely independent of each other (Blascovich, 2008; Blascovich \& Mendes; 2000). Consequently, it is not likely that there is a perfect match between the activation of familiarity and the complete evaluation of a motivated performance situation as "challenge." Familiarity is just one of its components and can interact with others in different ways. Moreover, as Blascovich (2008) claimed, familiarity being a factor that impacts the evaluation process, it may have implications for both resource and demand evaluation. Hence, familiarity may not only inform the presumably cognitive evaluation system of the availability of task resources, but also decrease task demands.

In order to test whether familiarity with a situation (manipulated by ways other than task-mastery) is more likely to generate challenge evaluations via its expected role on how 
information is processed (i.e., evaluated), it is necessary to create a performance situation in which it is possible to assess the activation of familiarity and its impact on processing. Recall that the FARM assumes that when individuals experience familiarity they process information on the basis of what they already know about the stimulus (Garcia-Marques \& Mackie, 2001) and not its specificities. Cognitive strategies that privilege mere activation of previous knowledge are defined by the FARM as only one of two ways that information can be processed; namely, non-propositional, non-integrative, non-analytic processing.

By testing the impact of familiarity on information-processing, one can validate the experience and assess its impact on the typical physiological response pattern associated with challenge. In addition, one can determine whether this physiological pattern is in some way (possible mediation) related to participants' engagement in a non-analytic processing mode, thus testing whether the decrease in analytic processing promoted by familiarity will be mediated by the challenge motivational state as measured by its physiological correlates. Assuming equal task demands in both familiar and non familiar conditions, it is hypothesized that the resource/demand evaluations resulting in challenge will stem in part from the evaluation of increase resources due to familiarity.

However, goal-relevance is necessary for task engagement and, only in that condition, is challenge expected to be differentiated from threat as a function of the resources and demands evaluation ratio. Task engagement is a necessary condition that influences cognitive processing directly. Evidence on dual process models has demonstrated that individuals who are task-engaged, whether because the issue is important (Petty, Cacioppo \& Goldman, 1981) or because they want to be accurate in the outcome of their judgment (Chen, Shechter \& Chaiken, 1998) tend to process information more analytically, than individuals who are not task-engaged. Task engagement is, therefore, conceived as a motivational variable that increases the likelihood of analytic processing (Petty \& Cacioppo, 1986). More relevant is the fact that such motivation seems to moderate the impact of familiarity in information processing. Low motivated performers are less likely to engage in more analytic processing and highly motivated individuals are much more likely to, independently of the level of familiarity (see Claypool et al., 2004). Thus, a moderated level of motivation would likely be a necessary condition to detect the regulatory role of familiarity. Such a moderated level should be sufficient to allow task engagement reported as necessary by Blascovich and colleagues. 
However, it should be noted that the definition of task engagement conveyed by the BPS model does not necessarily equate with motivation to process information analytically. According to Blascovich (2008), a situation is task-engaging if individuals perceive self- or goal-relevance; if it requires instrumental cognitive responses; and is active rather than passive. Even though self-relevance can increase analytic processing (Petty et al., 1991) perhaps it should not be confounded with the type of task engagement proposed by the BPS model. Consider the following example: when an individual has to deliver a speech in front of a video-camera (one of the most common ways to increase task engagement by BPS researchers), he/she can infer self or goal-relevance because his/her performance will be observed and performing well becomes important. However, this does not necessarily mean that his/her goal is to attend more carefully to the details of the information. The goal could be to better manage his/her self-image in other ways. So this motivation is not necessarily translated in a speech whose content is based on careful analytic processing, especially if an available response is offered and resources can be allocated to other features of the speech (e.g. body posture, tone, etc). So, in order to test the hypotheses here, it is necessary to use paradigm that increases the level of task engagement (in the BPS terms) sufficiently but without directly potentiating analytic processing. Furthermore, it is critical here to attend to the engagement factor.

\section{Overview of Experiments.}

Experiment 1. The general hypothesis was tested in Experiment 1. Specifically, it is hypothesize that those exposed to the familiar situation will process information nonanalytically and will exhibit the pattern of cardiovascular responses consistent with challenge.

The chosen paradigm involved: 1) testing the impact of familiarity on modes of processing; 2) measuring the relevant cardiovascular responses; 3) increasing task engagement (without increasing analytic processing). To do so, the paradigm used by Garcia-Marques and Mackie (2001, experiment 1) and Claypool et al. (2004) was adapted to suit these purposes. To that paradigm, a procedure used in other investigations of the BPS model (speech deliverance, Seery et al, 2004; Eliezer et al, 2010) was added that allowed task engagement while cardiovascular responses were being recorded. It was expected that the effects predicted by the FARM would be replicated and matched with the cardiovascular responses associated with a motivational state of challenge. 
Experiment 2. Experiment 2 tested a subsidiary hypothesis. Specifically, it is hypothesized that those for whom challenge motivation is increased will process information non-analytically.

Recall that the general hypothesis presupposes that the experience of familiarity is related to the experience of challenge, since both states are associated with the availability of resources to counter task demands. However, if challenge also informs the cognitive system of available task resources, then the hypothesis that challenged individuals' process information non-analytically arises. In other words, if the subjective experience signaling the availability of a response comes not only from familiarity but also from the engagement in a motivational state of challenge, then both experiences should produce similar effects on information-processing.

To test the independent effect of challenge on information-processing, the occurrence of familiarity within the experimental protocol was changed. Specifically, challenge and threat were induced prior to the exposition to the persuasive message. Congruent with the assumptions of the BPS model, evaluations of challenge and threat were manipulated by increasing or decreasing task-demands associated with an experimental task. It was expected that the manipulation of task-demands to exert the correspondent effect on the motivational states such that the non-demanding condition induced more challenge than the demanding condition. If challenge, as hypothesized, is able to affect information-processing in a way similar to familiarity, then one should expect a non-demanding condition to be associated with less analytic processing.

Note that "previous knowledge" like "repetition" has also (e.g. Cacioppo \& Petty, 1989) been associated with more analytic processing. However, Claypool and collaborators (2004) have shown that this effect is moderated by the level with which individuals engage in the task. Increases in analytic processing associated with repetition appear to be caused by additional opportunities to elaborate on message content (Cacioppo \& Petty, 1989; Cacioppo \& Petty, 1979) and previous knowledge with the ability of doing so (Wood, Kallgren \& Priesler, 1985). But either this opportunity or ability seems to be employed only when individual are motivated to deal carefully with the message. Because to promote the motivational states of challenge and threat individuals must be engaged in the task, it may be that both challenge and threat are clearly associated with analytic processing. However if task engagement has moderate levels and, as the FARM assumes, the evaluation of resources 
to deal with the task reduces the likelihood of analytic processing, it is hypothesized that challenge will be associated with non-analytic processing.

Because the assumption that the motivational state of challenge requires task engagement represents one of the differences between the FARM and the BPS models, it is crucial to focus on it directly. Note that the FARM model suggests familiarity to be activated and to influence individuals' processing with some, but not a special, engagement in the task. Moreover, if individuals are highly motivated to perform the task, such motivation can disrupt or even invert the role of familiarity. On the contrary, challenge appears to be activated under high task engagement conditions. As claimed above, task engagement should not be clearly identified as the general motivation associated with increased analytic processing. If it were, by increasing task engagement any impact of familiarity would be disrupted, though the effects of challenge and threat on information processing would be increased. If task engagement does not increase individuals' need for deep processing by itself, then it should not disrupt the familiarity effects on information processing. The reverse is not as clear because it is unlikely that individuals would be motivated to process deeply in a low engagement condition.

Although the previous hypotheses make task engagement point to the relevance of task engage in the studies here, this factor may further understanding of the relationship between challenge and a motivated performance situation in which familiarity is activated. If familiarity and challenge manipulations do not exert parallel effects under the same levels of engagement (as the differences between the two models seem to suggest), one can conclude that, although familiarity is incorporated into the motivational state of challenge, one is not reduced to the other. Thus, the approach in the subsequent experiments is to examine "task engagement" moderation effects.

Blascovich et al. (1999) have clearly demonstrated that the impact of familiarity on challenge is moderated by a factor that increases individuals' task engagement: the presence of others. When participants were observed by two confederates, they were more likely to be task-engaged. However, when participants were alone, task engagement was less likely and a clear differentiation between challenge and threat was not observed. So, as expected by the BPS model, examining the effect of familiarity on challenge is dependent upon task engagement, which may be achieved by the presence of others.

Because the presence of others increases task engagement, it should too moderate the challenge effects observed in Experiment 2 (challenge decrease analytic processing). Since 
challenge and threat motivational states are not differentiated with low engagement, it is predicted that these two states will not have differential impact in information processing in a lone (i.e., without observers) performance condition (where low engagement is expected). However, how information will be processed cannot be predicted. If low engagement means low motivation to process task information, then one would expect individuals to process information in a non analytic way. But if low engagement means moderated motivation, it is hypothesized that individuals would information analytically without observers.

If task engagement is necessary for the effects of familiarity on informationprocessing, then one should not find familiarity effects in an alone condition. However, this is not what is assumed by the FARM. Familiarity is expected to occur even without task engagement. Thus, unless the low engagement condition associates with a very low motivational state (which will engage participants by default in a non-analytic mode of processing), one would expect the observed familiarity effects on information-processing to be independent of the presence or absence of others. To test all these assumptions we conducted experiment 3 and 4.

Summary of Experiments 3 and 4. Experiment 3 tested the hypothesis that the impact of challenge on information-processing only occurs when task engagement is increased. This experiment was a simple replication of experiment 2 to which we added a manipulation of the presence vs. absence of others. Because the presence of others increases task engagement, we expected challenge to decrease analytic processing. But because task engagement is necessary to activate the motivational state, this effect on informationprocessing is not expected in the alone condition.

Experiment 4 tested the hypothesis that the impact of familiarity on informationprocessing will occur independently of task engagement. This experiment was a mere replication of experiment 1 to which we add a manipulation of presence vs. absence of others. If this variable does not influence the motivation for deep processing, allowing, therefore, a moderated level of motivation, we expect it will not moderate the effects of familiarity in how information is processed. 
Section II: 


\section{Experiment 1}

This experiment was designed to test the general hypothesis that the impact of familiarity on information-processing hypothesized by Garcia-Marques et al's (2000; 2001; Claypool et al, 2004; Moons et al, 2009) theory and research is associated with Blascovich's (2008) motivational state of challenge. The experimental design was based on the assumptions of both the FARM and the BPS models. It was modeled on Garcia-Marques and Mackie's (2001) and Claypool et al.'s (2004) investigation of persuasion.

This design allowed tests of the impact of familiarity on the activation of information-processing modes. More specifically, it permitted assessment of depth of processing by asking individuals to process either strong or weak persuasive messages, under either familiar and unfamiliar conditions, and rate their opinions of the messages and subsequently observing attitude-change. According to Petty and Cacioppo (1986), strong messages contain arguments such that when individuals are instructed to think about the message, their generated thoughts are predominantly favorable. Positive attitude-change occurs when individuals generate favorable thoughts towards the position defended in the message. According to Petty and Cacioppo, weak messages contain arguments such that when individuals are instructed to think about the message, they generate predominantly unfavorable thoughts.

Congruent with well-established persuasion models (e.g. Elaboration Likelihood Model, Petty \& Cacioppo, 1986; Heuristic-Systematic Model, Chaiken, 1987), when an individual is elaborating message content, strong and weak arguments have differential impacts on attitude-change. In these situations, individuals generate thoughts that are congruent with the quality of the message, such that, strong messages typically lead to more favorable thoughts, and weak messages to more unfavorable ones. In contrast, when individuals are not elaborating, strong and weak arguments have a similar impact on attitude-change and the thoughts they generate (usually fewer in comparison to elaboration) are independent of message quality.

Because the cardiovascular indexes of challenge and threat have only been validated during motivated performance situations, i.e., situations that are task-engaging, task engagement needed to be induced without simultaneously maximizing analytic processing. Consequently, a task was developed that simultaneously would induce task engagement and be informative about depth of information-processing. Following earlier investigations of the 
BPS model (e.g. Seery et al, 2004; Eliezer et al, 2010), task engagement was induced by requiring participants to give a speech recorded via a video camera and microphone while their cardiovascular responses were recorded. They were instructed to give a speech based on the thoughts elicited by a persuasion message that was previously presented to them. This task allows, via the content of the speeches, coding of participants thoughts as they read the persuasion message informing us about depth of processing (Greenwald, 1968; Tormala, Briñol \& Petty, 2006).

This paradigm developed in three different phases. The first phase served the purpose of inducing familiarity based on the manipulation of familiarity used by Claypool et al. (2004). Participants read either a strong or weak persuasive message on a computer screen while they listened to an audio message that would be subsequently repeated or not. The second phase served the purpose of testing the impact of familiarity on informationprocessing. Participants read either a strong or weak version of a persuasive message, which for half of the participants was the same message they heard in the first phase. The third phase, because it increased task engagement, allowed the assessment of the association between familiarity activated by different familiarity conditions with the cardiovascular markers of motivational states of challenge usually associated with those states. Task engagement was induced by asking participants to give a speech to a video-camera (Seery et al, 2004; Eliezer et al., 2010) about the thoughts they were having while they were reading the persuasive message previously. Physiological recordings were made throughout the three tasks.

Because the FARM model postulates that repetition of information decreases the probability of analytic processing, replication of the previous results by Claypool et al. (2004) was expected on phase 2. Specifically, it was hypothesized that participants' attitudes in the familiarity condition would be independent of the quality of the arguments whereas attitudes of those not in the familiarity condition would reflect the quality of the arguments. In addition, it was hypothesized that the favorability of the thoughts (towards the issue presented in the message) generated by participants in phase 3 would be moderated by familiarity conditions. More specifically, it was hypothesized that in the familiarity condition that favorability would be independent of message quality and would anchor the quality of the message for those not previously familiar with the message. More specifically it was hypothesized that those in the familiar condition would report the same number of thoughts, independently of the message quality, reflecting superficial processing. In contrast, for those 
in the unfamiliar condition, the number of thoughts was expected to differ as a function of message quality.

The main test of the general hypothesis centered on the impact of familiarity on challenge state. Recall that it was hypothesized that because familiarity increases the likelihood of challenge, both states are associated with the availability of resources to deal with task demands. Hence, it was hypothesized that during phase 3 those in the familiarity condition would exhibit a pattern of cardiovascular responses consistent with challenge increases in cardiac activity (HR and VC) and increases in CO and decreases in TPR. In addition, because this pattern of results may suggest a close relationship between familiarity activated in the repetition condition and the challenge motivational state as indexed by these physiological markers, we will address them (the physiological markers) as a mediation variable of the effect of familiarity on information processing mode

\section{Method}

\section{Participants and Design}

48 undergraduate students from the University of California, Santa Barbara (UCSB; 34 females; Mean age $=20 ; S D=1.94$ ) received course credit for their participation. Participants were randomly assigned to one of the conditions in the 2 (strong vs. weak message) x 2 (repetition $v s$. no-repetition) between subjects factorial design.

\section{Material}

The experimental material consisted of two persuasive messages adapted from Garcia-Marques and Mackie (2001). One argued for the effectiveness of weight loss centers; and the other argued against imposing restrictions on industry to minimize the effects of acid rain on the north eastern states of the USA. Each message had a strong and weak version comprised of strong and weak arguments, respectively. The strong weight loss center message consisted of arguments such as "Weight loss centers are monitored full time by qualified medical professionals.", whereas the weak version consisted of arguments such as "Attending a weight loss centre program weekly meeting is a really good and healthy way to spend time and meet new people." The strong version of the acid rain message included arguments such as "Solving demographic and geographic problems would have a more 
beneficial effect than imposing controls on industry to minimize the effects of acid rain"; whereas the weak version included arguments such as "Everyone is always blaming American industry for everything, and using them as an excuse to increase bureaucracy." (see appendix A to see full messages).

Original pretests performed by Worth and Mackie (1987) revealed that these messages were likely to be counterattitudinal to the majority of UCSB students; and that both strong versions of the messages were more persuasive than the weak versions. An additional message was used as a control for the manipulation of familiarity. This message argued against increases in road taxes with a strong version (e.g. "An increase in tax rates (...) would be used to repair potholes in our freeways which play a part in $38 \%$ of all highway traffic deaths every year in America.) and a weak version (e.g. "Tax rate increases would help in the beautification of our freeways and also make them more interesting.") (see appendix A).

The materials for this experiment also included equipment (i.e., sensors, and transducers) necessary to record participants' physiological responses and obtain the physiological recordings (see Blascovich Mendes, Vanman, \& Dickerson, 2011, chapter 2, for details).

\section{Procedure}

Participants arrived singly at the laboratory where they were greeted by an experimenter and read an "informed consent" for explaining the nature of the experiment. After obtaining their informed consent participants completed an initial questionnaire that included demographic questions (gender, class, age, deafness or difficulty in hearing or speaking) as well questions regarding their opinions and feelings towards statements reflecting different attitudinal issues (see appendix B for the complete questionnaire). Examples of these attitudinal issues were recycling ("The government should be stricter with people who don't recycle"), pollution ("Smog checks on automobile have been effective in reducing air pollution") or smoking ("Anti-smoking programs have been effective in preventing cigarette addiction"). In addition to these, individuals' attitudes towards weight loss centers ("Weight loss centers are places where people can safely and effectively lose weight") and acid rain ("The government should impose controls on industry to help minimize the effect of acid rain in the US") were also assessed. These were key statements 
because they provided pre-attitudinal measures of the topics that were subsequently subject to persuasive counter arguments during tasks 1 and 2 .

Participants responded via a "feeling thermometer" (see appendix B). As in GarciaMarques and Mackie's experiment (2001), participants were instructed to read each statement and use the thermometer to express how "cold" or "hot" they felt about the topic. The thermometer's scale ranged from 0 to 100 . Participants were informed that if they disagreed with the statement, they could give it a "cold" rating, choosing a temperature somewhere between 0 and 49; but if they agreed with it then they could give it a "hot" rating somewhere between 51 and 100. If they were neutral then they could give it a 50 .

Next, the appropriate physiological sensors were applied to participants' torsos and necks in accordance with the guidelines provided by the Society for Psychophysiological Research (e.g., Sherwood, Allen, Fahrenberg, Kelsey, Lovallo, \& van Dooren, 1990) and in accordance with hospital and commercial safety standards.

Physiological signals were recorded using a Biopac impedance cardiograph (Model NICO100C), a NIBP100A blood pressure monitor and a Biopac electrocardiograph amplifier (Model ECG100C). Electrocardiographic (ECG) and impedance cardiographic (ZKG) recordings provided continuous measures of cardiac performance. Employing a tetrapolar aluminum/mylar tape electrode system, impedance cardiography provides basal transthoracic impedance (Z0) and the first derivative of basal impedance (dZ/dt). Two pairs of ZKG tape electrodes were fastened around the participants' necks and torsos. A $400 \mu \mathrm{A} \mathrm{AC} 50 \mathrm{kHz}$ current is passed through the top and bottom electrodes and basal impedance is measured via the inside electrodes. ECG recordings were attained using a modified lead II configuration (lower left torso and upper right torso with impedance cardiography providing an internal ground). Continuous, noninvasive blood pressure measurements were obtained using a NIBP100A blood pressure monitor that uses a pressure sensor placed on the wrist over the radial artery. This device uses a "sweep technique," which applies a varying force on the radial artery. The counter-pressure in the artery produces a signal which is digitized and used to calculate blood pressure parameters. Finally, data were integrated with an MP150 and displayed and stored with Acknowledge software (Biopac, Goleta, CA). We used Mindware software to edit artifacts and ensemble and score the data.

After applying the physiological sensors, participants were left alone for 5 minutes so that baseline recordings of their cardiovascular responses could be collected. Next, the experimenter returned and stopped the recording of the physiological data. She instructed 
participants that the goal of the experiment was to understand how people perform multiple tasks on a computer while they are attached to physiological equipment. They were further informed that all the instructions necessary to perform each task would be presented at the appropriate time via the computer. They were instructed that all they had to do was read the instructions carefully and carry out the different tasks in order. Following these general instructions, the experimenter started the Medialab-based software on a PC computer that randomly assigned participants to one of the four experimental conditions. Next, she restarted the physiological recordings, gave instructions to begin the experiment, and sat quietly out of view on the opposite side of the room. Physiological readings were recorded throughout the rest of the experiment.

Initially, participants read a brief review of the goal of the experiment followed by instructions for performing the first task. As in Claypool et al.'s study (2004, experiment 2), all participants were informed that the upcoming task involved performing simultaneous activities. Before the task began, all participants were instructed to put on provided headphones and press the space bar to initiate. After they complied, they were instructed that they should read a message on the computer screen while listening to a separate background message designed to create a regular environment like studying at a coffee shop while listening to background music. The instructions stressed that their main and most important goal was to read the message on the screen carefully and to ignore the background message.

All participants read the randomly assigned strong or weak message arguing for the effectiveness of the weight loss centers. Those assigned to the no-repetition condition heard a message arguing for an increase in road taxes. Those assigned to the repetition condition heard a message arguing against imposing restrictions on industry to minimize the effects of acid rain. The strength of the written message was matched to the strength of the audio message so that participants who read a strong message about the weight loss centers also listened to a strong message about road taxes/acid rain and vice-versa. The average time to read the message matched the length of the audio message so that participants would not concentrate on the audio message after the reading the screen message.

After the message presentations, participants expressed their agreement with three items related to the effectiveness of weight loss centers ("Weight loss centers are places where people can safely lose weight”; "Weight loss centers offer good support to those who want to lose weight"; and "Weight loss centers do not offer ways of efficiently losing weight”). The items were presented successively and each item was accompanied by a 7- 
point rating scale (where $1=$ total disagreement; and $7=$ total agreement). To give their responses, participants pressed the computer key with the number that best corresponded to their opinion.

For the second task, participants read another set of instructions. They were informed that the new task required only reading a message and, therefore, they could remove the headphones. Again, participants were instructed to read the message carefully. Participants read a randomly assigned strong or weak message arguing against imposing restrictions on industry to control the effects of acid rain. For those in the repetition condition, this message was the same acid rain message played over the headphones in the previous task. For participants in the no-repetition condition, this was their first exposure to this message. After reading it, participants expressed their agreement towards the position defended in the message in three successively presented statements ("The government should impose controls on industry to help minimize the effect of acid rain in the US; "Increases in problems with acid rain in the US should not be blamed on the activities of industries operating in affected areas"; "The government should require the installation of sulfur dioxide emissions control devices in factories operating in the US"), anchored in a 7-point rating scale (where $1=$ total disagreement; and $7=$ total agreement).

Next, new instructions informed all participants to think about the thoughts they had while reading the acid rain message because they were about to give a speech based on those thoughts. They were further told that the speech would be recorded via video-camera. Participants were given one minute to prepare the speech and three minutes to deliver it. Before they start the task, the experimenter set up the video-camera. Then, she instructed them to start the preparation minute by pressing the space bar and went back to her seat. After the minute was over, instructions on the screen informed participants to start delivering the speech.

After three minutes, instructions appeared on the screen informing participants that the experiment was over. Subsequently, the experimenter turned off the physiological recording equipments and removed the sensors from the participant's body. Finally, participants received a final questionnaire designed to assess relevant control measures, like the effectiveness of the familiarity manipulation, participants' mood, challenge and threat evaluations or motivation to perform the task (see appendix $\mathrm{C}$ for complete questionnaire). In the end, participants were fully debriefed and thanked for their participation. 


\section{Dependent Measures}

Attitude-Change Measures. Attitude-change regarding weight loss centres and acid rain were assessed as described above via three items anchored on 7-point rating scales. Factor analysis on the three items regarding weight loss centers revealed a single-factor solution (explaining more than $50 \%$ of the total variance). A calculation of reliability revealed a Cronbach's $\alpha=.41$. Similarly, a factor analysis to the three items regarding acid rain revealed a single-factor solution (explaining more than $73 \%$ of the total variance) and a Cronbach's $\alpha=.82$. Based on the factor analysis, the ratings on each message were averaged and a composite measure was created as the post attitude-change measure. Finally, this value was subtracted from the thermometer-based pre-attitude (rescaled to a 0 to 7 ) such that ratings from 1 to 14 on the thermometer were scaled as 1 ; ratings from 14 to 28 were scaled as 2; ratings from 28 to 42 as 3; ratings from 42 to 58 as 4 ; ratings from 58 to 72 as 5; ratings from 72 and 86 as 6; and ratings from 86 to 100 as 7) which provide us with an attitudechange index for both messages.

Thought Measures. The number of thoughts generated by each participant and their favourability towards the position promoted by the message served as a measure of information-processing depth during the motivated performance situation.

Challenge and Threat Indexes. Reactivity scores (i.e., changes from baseline levels) for Heart Rate (HR), Ventricular Contractility (VC), Cardiac Output (CO) and Total Peripheral Resistance (TPR) were calculated and served as indexes of challenge and threat states. The EKG and ZKG signals were scored using an interactive software program (MindWare) that produces ensemble averaged values for the cardiac inotropic measures (e.g., preejection period; PEP) and cardiac chronotropic measures (e.g.,HR). This served as the minute-based data for all the indexes. The ensemble averaging technique effectively eliminates respiratory and movement artifacts from continuously recorded signals (Kelsey \& Guethlein, 1990).

Data was ensemble by first finding cycle peaks within intervals of 60 seconds. Then, an average peak was selected. We highlighted to the trough before peak to find delta $\mathrm{T}=\mathrm{PEP}$. Then, we highlighted from the highest slope value to middle of trough on dzdt to find deltaT=LVET. Finally, we highlighted the top of $\mathrm{dz}$ peak to find dzmin. HR was scored by ensemble averaging the number of times the heart beat within intervals of 60 seconds. VC 
was calculated by multiplying pre-ejection period (PEP) by -1 , where PEP represents the time in milliseconds in the cardiac cycle from initiation of ventricular depolarization to opening of the aortic valve and ejection of blood. Pre-ejection period was scored manually from ensemble-averaged EKG and ICG waveforms. CO was calculated by multiplying HR by Stroke volume, i.e., the volume of blood, in milliliters, pumped out of the heart with each beat. Finally, TPR was calculated by dividing mean arterial pressure (MAP) by cardiac output and multiplying the total by 80 (Sherwood, Allen, Fahrenberg, Kelsey, Lovallo, \& van Dooren, 1990).

Mean values of $\mathrm{HR}, \mathrm{VC}, \mathrm{CO}$ and TPR were calculated for every minute of the baseline and the speech task. Then, reactivity scores for each CV response were calculated by subtracting the $5^{\text {th }}$ minute of the baseline, where physiological rest is higher, by the $1^{\text {st }}$ minute of the speech task, where physiological activity is higher (Mendes et al, 2008).

\section{Results and Discussion}

\section{Attitude-Change Data.}

Effectiveness of the familiarity manipulation. The manipulation of familiarity was corroborated by and ANOVA procedure. Specifically, participants' ratings on the item "The presentation of the audio message on phase 1 made the second written message more familiar", entered in a 2 (repetition vs. no-repetition condition) x 2 (strong vs. weak message) ANOVA, revealed a main effect of repetition, $F(1,44)=11.85 ; p=.001 ; \eta^{2}=.20$. Participants in the repetition condition considered the presentation of the message more familiar $(\mathrm{M}=5.25 ; \mathrm{SD}=0.33)$, than those in the no-repetition condition $(M=3.67 ; S D=$ $0.33)$. The message quality had neither a direct impact on the measure, $F(1,44)=1.61 ; p=$ .21 , nor did it moderated the impact of repetition, $F<1$ (see appendix D).

Attitude-Change towards the Weight loss center message. Because instructions stressed careful processing of the message content to all participants, it was expected that message quality would affect participants such that they would be persuaded more by the strong than by the weak message independently of the experimental condition regarding repetition of the message. The attitude-change index on this the subject (i.e., weight loss centers) for each participant were analyzed via a 2 (repetition vs. non-repetition condition) $\mathrm{x}$ 
2 (strong vs. weak message) ANOVA. As hypothesized, a marginal main effect of message quality on attitude-change was revealed, $F(1,44)=3.71 ; p=.06 ; \eta^{2}=.08$. Participants agreed with the claim more following exposure to the strong version of the message $(M=$ $1.47 ; S D=0.22)$, than exposure to the weak version $(M=0.86 ; S D=0.22)$. No other main effect or interactions were found, $F^{\prime} s<1$. The lack of interaction between repetition and argument quality suggests that all participants engaged (as expected) in more deep, analytic processing (see appendix D).

Attitude-Change towards the Acid Rain Message. In order to test the impact of familiarity in reducing the likelihood of analytic processing, the difference between participants' reactions to strong and weak versions of the message were analyzed. This acid rain attitude-change index was used as a dependent measure in a 2 (strong vs. weak message) x 2 (repetition $v s$. no-repetition condition) ANOVA.

Previous results were replicated showing the presence of the hypothesized two-way interaction, $F(1,44)=5.23 ; p=.03 ; \eta^{2}=.11$. As expected, the interaction was driven by a familiarity moderation of the impact of strong and weak arguments on attitude-change, which is present in the no-repetition condition but not in the repetition condition (see Figure 1). Contrast analysis revealed that whereas those who read the message for the first time were significantly more persuaded by the strong than by the weak message, $t(23)=2.61$; $p=$ $.01 ; \eta^{2}=.23$, those in the repetition condition reacted equally to strong or weak arguments $t<1$. No main effects were found either for argument quality, $F(1,44)=1.97 ; p=.17$, or for the familiarity factor, $F<1$. These results replicate the previous findings in the FARM model (e.g. Garcia-Marques \& Mackie, 2001) by demonstrating that repetition decreased analytic processing (see appendix D). 


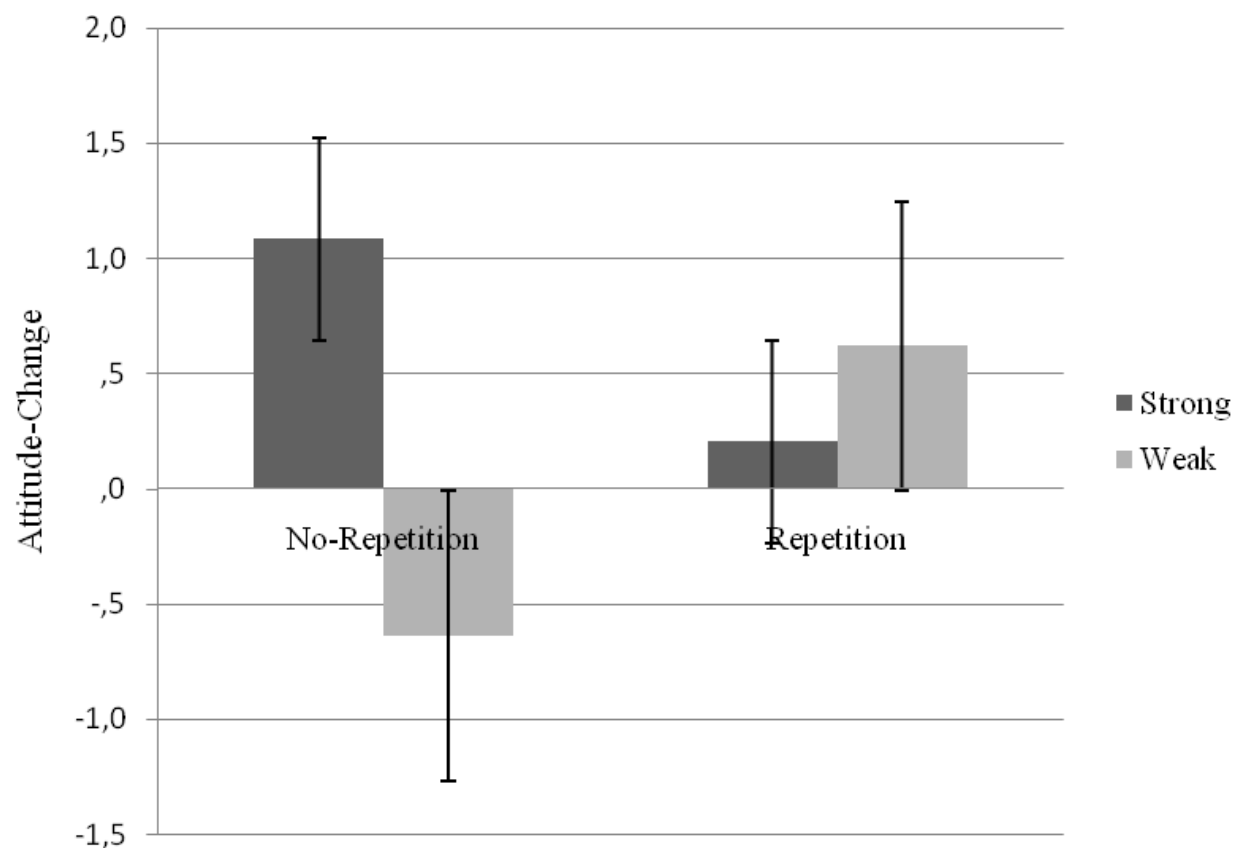

Figure 1. Attitude-change index towards the target issue as a function of message repetition and argument quality.

\section{Thoughts Data.}

Using number of thoughts as a measure of elaboration (i.e. more thoughts if participants engage in analytic processing, e.g. Petty \& Cacioppo, 1986), it was hypothesized that repetition would impact this measure in such way that those in the repetition condition would generate fewer thoughts than those in the no-repetition condition. To test this hypothesis, the number of thoughts generated by each participant was entered in a 2 (strong vs. weak message) $\mathrm{x} 2$ (repetition vs. no-repetition condition) ANOVA. Results revealed the expected main effect of repetition, $F(1,44)=4.59 ; p=.04 ; \eta^{2}=.09$. Congruent with results presented above, those in the repetition condition generated fewer thoughts $(M=1.64 ; S D=$ $0.14)$ than those in the repetition condition $(M=2.07 ; S D=0.14)$. No other effect was significant, $F^{\prime} s<1$.

Additionally, it was hypothesized that attention to the quality of the message would impact the favorability of thoughts, such that strong messages would be associated with more favorable thoughts and the weak message with more unfavorable thoughts. Because only those in the no-repetition condition were expected to attend carefully to the quality of the message, familiarity was hypothesized to qualify this effect. To test this hypothesis, three 
independent judges were asked to code each thought as favorable, unfavorable or neutral towards the position defended by the message (Cohen's Kappa $=.83$ ). Subsequently, the proportion of favorable and unfavorable thoughts as repeated measures were analyzed via a 2 (favorable vs. unfavorable thoughts) x 2 (strong $v s$. weak arguments) x 2 (repetition $v s$. norepetition) ANOVA.

Results revealed that, in general, favorable thoughts were generated more $(M=0.42$; $S D=0.05)$ than unfavorable ones $\left.(M=0.27 ; S D=0.03), F(1,44)=4.77 ; p=.03, \eta^{2}=.10\right)$ and that the type of thoughts differed as a function of the message quality, $F(1,44)=15.91 ; p$ $<.001 ; \eta^{2}=.27$, such that, more favorable thoughts were generated after the strong message $(M=0.42 ; S D=.04$ vs. Munfavorable $=.29 ; S D=.07)$ and more unfavorable thoughts were generated after the weak $(M=0.54 ; S D=.07$ vs. Mfavorable $=.12 ; S D=.04)$.

As predicted, this interaction was moderated by familiarity, $F(1,44)=4.34 ; p=.04$, $\eta^{2}=.09$. As shown in table 1 , the number of favorable thoughts differed as a function of message quality in the no-repetition condition, $t(44)=4.29 ; p<.001 ; \eta^{2}=.29$, but not in the repetition condition, $t(44)=-1.34 ; p=.18 ; \eta^{2}=.04$. This effect was primarily due to a familiarity $\mathrm{x}$ type of thoughts interaction, $F(1,44)=17.47 ; p<.001 ; \eta^{2}=.28$. Whereas the thoughts of participants in the repetition condition were independent of the message quality, the thoughts of participants in the no-repetition condition were more favorable when the message was strong but unfavorable when the message was weak (see appendix D).

\begin{tabular}{ccccc}
\hline \multicolumn{2}{c}{ No-Repetition } & \multicolumn{2}{c}{ Repetition } \\
\hline Type of & Strong & Weak & Strong & Weak \\
Thought & & & & \\
\hline Favorable & $.69(0.05)$ & $.18(0.06)$ & $.14(0.06)$ & $.06(0.05)$ \\
Unfavorable & $.14(0.10)$ & $.46(0.10)$ & $.44(0.10)$ & $.63(0.10)$ \\
\hline
\end{tabular}

Table 1. Proportion of favorable and unfavorable thoughts as a function of message repetition and message quality.

Given that favorability of thoughts was associated with both strong and weak messages reflecting the degree of elaboration and thus of analytic processing, it was hypothesized that favorability would mediate the impact of familiarity on processing. Because individuals in the no-repetition condition generated thoughts that were dependent 
on the quality of the message and, in turn, the favorability of the thoughts they were having while processing the persuasion message was hypothesized to mediate the effects of argument quality on attitude-change (Greenwald, 1968). To test this hypothesis, an index of thought favorability was created for each participant by subtracting the number of unfavorable thoughts from the number of favorable thoughts and dividing the difference by the total number of thoughts (Tormala, Briñol \& Petty, 2006). Next, this value was entered as predictor in a regression analysis model, in which the acid-rain attitude change-index served as the dependent variable and the message quality as predictor. Results revealed that the thought favorability index totally mediated the impact of message quality in the norepetition condition, given that the impact of message quality on attitude-change $(b=.58$, $t(22)=3.30, p=.003)$ was eliminated when the thought favorability index $(b=.26, \mathrm{t}(22)=$ $1.21, p=.24$ ) was controlled. For the repetition condition, the thought favorability index neither predicted attitude-change, nor mediated the effects of message quality on attitudechange, $t^{\prime} s<1$ (see appendix D).

\section{Challenge and Threat Data.}

The main hypothesis focused on different motivational states associated with familiarity and non familiarity conditions. In order to assess these states, the physiological markers of the motivational state were analyzed based on previous analytic strategies (e.g. Mendes et al, 2008; Weisbuch-Remington et al, 2005). First, baseline differences were tested; next task engagement was assessed; and lastly the challenge and threat indexes were analyzed as a function of the experimental manipulations.

Baseline Differences ${ }^{1}$. No baseline differences were found by experimental condition for the $5^{\text {th }}$ minute of HR, VC, CO and TPR values as dependent measures in a 2 (strong vs. weak message quality) x 2 (repetition vs. no-repetition condition) MANOVA and no main effects or interactions emerged, $F^{\prime} s<1$ (see appendix D).

Task engagement. It was assumed that individuals would be task engaged in the speech task as revealed by increases from baseline in HR and VC values. This hypothesis was confirmed. Both HR and VC reactivity indexes differed significantly from zero using

\footnotetext{
${ }^{1}$ We tested possible differences between the $4^{\text {th }}$ and the $5^{\text {th }}$ minute of the baseline values in all our studies and observed no differences. This indicates that in all the studies participants had reached baseline resting values. As such, we used the $5^{\text {th }}$ minute of the baseline as our baseline measure in all the studies.
} 
independent t-tests, HR: $t(47)=2.48 ; p=.02 ; \eta^{2}=.12(M=15.09 ; S D=42.16)$; VC: $t(47)=$ $4.93 ; p<.001 ; \eta^{2}=.34(M=10.59 ; S D=14.89)^{2}$ (see appendix D).

Challenge and Threat indexes. In order to test the hypothesis that familiarity would increase the pattern of responses associated with challenge, CO and TPR reactivity indexes were entered as dependent measures in two separate 2 (strong vs. weak message) $\mathrm{x} 2$ (repetition $v s$. no-repetition condition) ANOVAs. Results corroborate the presence of a main effect of familiarity on both TPR, $F(1,44)=4.02, p=.05 ; \eta^{2}=.08$, and CO, $F(1,44)=5.51$, $p=.02 ; \eta^{2}=.11$. As hypothesized, the challenge pattern emerged for the repetition condition such that participants in this condition exhibited a decrease in TPR and an increase in CO (see Figure 2). These results corroborate and extend previous work on the BPS model (e.g. Blascovich et al., 1993) by demonstrating that familiarity by itself, without necessarily promoting mastery, is associated with a motivational state of challenge. Message quality did not interact or have any other effect on these indexes $\left(F^{\prime} s<1\right)$ (see appendix D).

TPR

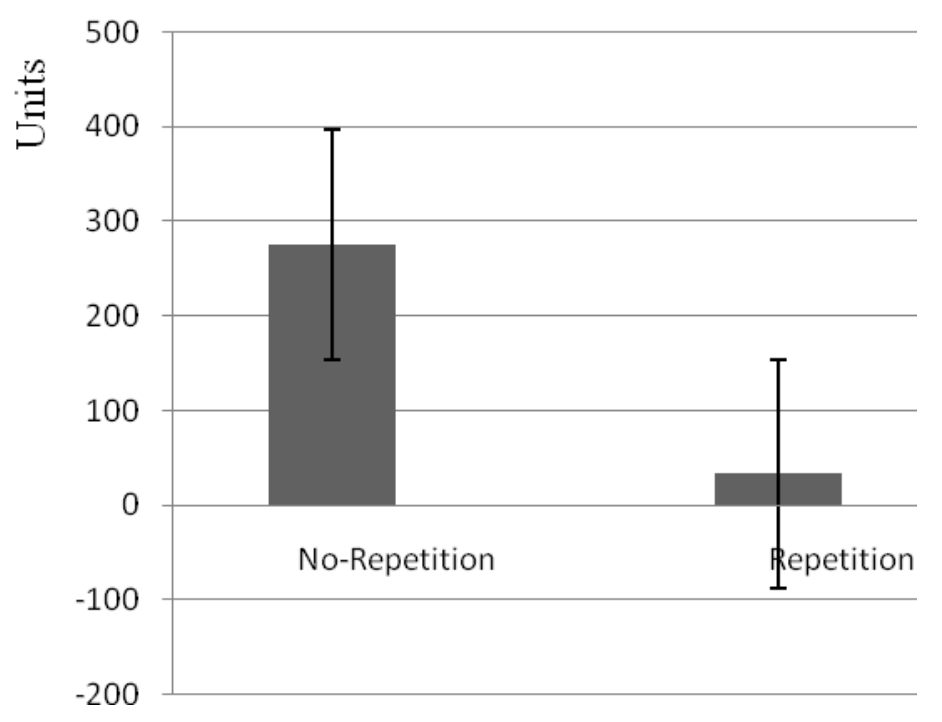

\footnotetext{
${ }^{2}$ We also tested if participants were task-engaged while they were reading the acid-rain message and no significant effects emerged. Both $\mathrm{HR}$ and $\mathrm{VC}$ were not different from $0, t$ 's $<1$. This comes as no surprise since the BPS model postulates that task engagement is not likely to occur under passive tasks, like reading a text or watching a video.
} 


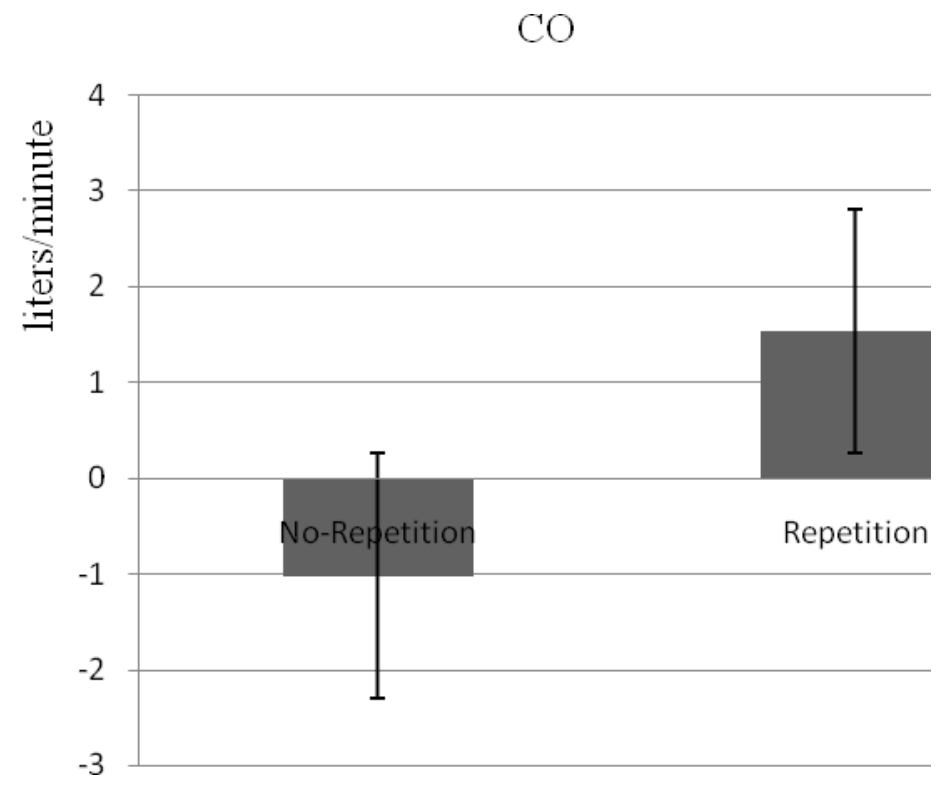

Figure 2. TPR and $\mathrm{CO}$ values during the speech task as a function of message repetition.

These results confirm the general hypothesis that familiarity impacts the type of processing in which participants engage and, is also associated with the motivational state of challenge. Those in the repetition condition who had processed information superficially also were challenged. A straightforward interpretation of these results suggests that the cognitive system is informed about resource availability, which was evaluated by participants in a repetition condition.

Congruently, it was hypothesized that the impact of familiarity on informationprocessing could be in some way (possible mediation) related with participants' engagement in a challenge motivational state. This was tested by determining if the decrease in analytic processing promoted by familiarity was mediated by the challenge state, using the physiological correlates. Specifically, the hypothesis that the cardiovascular markers mediate the FARM effects was tested.

In order to do so, a unitary index of challenge and threat was created by converting each participant's $\mathrm{CO}$ and TPR values into z-scores and summing ZCO with ZTPR (cf. Blascovich et al., 2004). More positive scores in this index indicate challenge and less positive or negative scores indicate threat. However, the introduction of the unitary index as a covariate in the 2 (strong vs. weak) x 2 (repetition $v s$. no-repetition) ANOVA model with the acid-rain attitude change index as the dependent measure did not confirm the mediation 
hypothesis. This hypothesis is ruled out by data demonstrating that the covariate has no impact on the dependent measure $F<1^{3}$ (see appendix D).

One possible explanation has to do with the fact that assessment of attitude and physiological measures occurred made in different phases of our study. Hence, the information-processing data of the speech task (where task engagement was triggered and challenge and threat were differentiated) was analyzed to determine if engagement in motivational state mediated the effects of familiarity on the number of thoughts, our measure of elaboration. Consequently, the unitary challenge and threat index was entered as a covariate in the 2 (strong $v s$. weak) x 2 (repetition $v s$. no-repetition) ANOVA model using the number of thoughts (our measure of elaboration) as dependent measure. Again, results did not found evidence for a main effect of the covariate $F<1$ which rules out the possibility of mediation $^{4}$ (see appendix D).

Given engagement in a state of challenge or threat is moderated by one's evaluations of resources and demands (being challenge activated when participants evaluate more resources than demands), the possibility of mediation via these evaluations was tested by entering participants' responses on the item "In general, I think I had sufficient capacity to deal with what was demanded by the task" as covariate in the 2 (strong vs. weak) x 2 (repetition vs. no-repetition) ANOVA model with the acid-rain attitude change index as the dependent measure. Once more, the possibility of mediation is ruled out by the absence of a significant impact of the covariate on the dependent measure, $F<1$. Similarly, when the number of thoughts was analyzed as a dependent measure, the main effect of the covariate was not significant, $\mathrm{F}<1$. Hence, these results also ruled out the possibility of mediation by reported evaluation of availability of resources (see appendix D).

Because challenge is generally (but not always) defined as a positive hedonic state (Blascovich \& Mendes, 2000), the possibility of mediation via mood assessment was tested. The three items assessing mood (How do you feel right now? 1-Sad; 7 - Happy; How do you feel right now? 1 - Discontent; 7 - Content; How would you describe your mood at this time right now? $1-\mathrm{Bad} ; 7-\mathrm{Good}$ ) were averaged in a single measure (explaining more

\footnotetext{
${ }^{3}$ We also tested the possibility of mediation using the TPR and CO raw values as covariates in the ANOVA model. The results also rule out the possibility of mediation since the main effect of both TPR and CO on the dependent measures were not significant, $F^{\prime} s<1$.

${ }^{4}$ Neither TPR, nor CO had a main effect on this dependent measure when they were introduced in the ANOVA model, $F^{\prime} s$ $<1$.

${ }^{4}$ Neither TPR, nor CO had a main effect on this dependent measure when they were introduced in the ANOVA model, $F$ ' $s$ $<1$.
} 
than $85 \%$ of the total variance and a Cronbach's $\alpha=.91$ ) that was subsequently entered as covariate in the 2 (strong $v s$. weak) x 2 (repetition $v s$. no-repetition) ANOVA model with acid-rain attitude change index as the dependent measure. The results revealed that the mood had no direct impact on the dependent measure, $F(1,43)=1.20 ; p=.28$. Similarly, with the number of thoughts as dependent measure, mood had no direct impact, $F(1,43)=2.12 ; p=$ .15 (see appendix D). These results rule out the possibility of mood mediation.

From these data, it can be concluded that the impact of familiarity on informationprocessing occurs in a situation in which cardiovascular responses reveal a motivational state of challenge. However, the effect does not seem to be dependent upon it, at least in any straightforward way. Familiar conditions are theoretically associated with a physiological response typically associated with challenge but the FARM effects seem to be independent of that state as neither the cardiovascular markers, nor the covert cognitive evaluations of challenge or mood mediated the effects.

These data suggest that although a feeling of familiarity promotes less analytic processing, and familiar conditions promote physiological responses usually associated with challenge, the two "measures" are independent. They do not appear anchored in the same process, that is, the information of resource availability for performing a task. This conclusion is however problematic because those "measures" were relied upon as highly valid measures of resource availability. But, recall that both effects of familiarity on processing and on physiological measures are not pure measures of the variable that promote both effects.

For some reason, they may assess different features of the same antecedent and although they may have the same source variable, they may not be correlated. Additionally, the signal of resource availability given by a non-necessarily evaluated feeling of familiarity may not be totally incorporated into the evaluation process. Moreover, it is relevant to remember that physiological measures were assessed at a different time than the attitudes and so both could have been influenced by other sources of variability during those times. Even if this source of influence did not prevent the detection of an influence of familiarity, they could have something less than a pure relation with other source. In addition, recall that the physiological measures are only markers of the motivational states and as such do not necessarily and fully index them. They are, for example, able to be activated by other sources. Tomaka et al. (1993) have demonstrated that the exhibition of the different 
cardiovascular patterns is not causally linked to the cognitive evaluations of resources and demands which determine the activation of challenge and threat. For example, they found that asking individuals to immerse their hands in a warm bath mimics the challenge cardiovascular responses but that effect is independent of the cognitive resources and demands evaluations.

Another possible explanation for our data that does not allow a direct dismissal of the resource evaluation hypothesis associated with familiarity, is associated with the fact that challenge is a compound ratio index of resources and demands. Two possibilities should be considered. First assuming that familiarity impacts evaluation of "resource availability" it is not highly likely to find a perfect map between the activation of familiarity and the complete evaluation of a situation as "challenge", because it is just one of its many possible components. But it is also possible that familiarity promotes a physiological response pattern associated with challenge towards also other evaluation components. It may simply suggest at the same time that the task was "easier" and thus, may have impacted the demand features of the task. For these reasons, examinations of the relationship between familiarity and challenge were continued albeit using different approaches in order to further test the mediation hypothesis.

\section{Experiment 2}

Assuming that challenge, like a feeling of familiarity, informs the cognitive system of resources available to deal with task demands, it was hypothesized that challenged individuals would process information more superficially. The goal of this experiment was to test the hypothesis that the activation of a motivational state of challenge impacts information-processing in the same way as a manipulation of familiarity does it. As observed in experiment 1 , repetition reduced analytic processing. Thus, in this experiment it was hypothesized that challenge would have the same effect.

Challenge and threat were manipulated via perceptions of task demands. Congruent with the BPS model (e.g. Tomaka \& Blascovich, 1996; Blascovich, 2008), evaluations of task demands interfere with the cognitive evaluations of resources and therefore motivational state. If a task is demanding, individuals usually evaluate fewer resources than the ones required by the situation. In this case, motivational threat is most likely activated. If a task is 
not demanding, individuals usually perceive enough or more resources to cope with the task demands and will likely engage in motivational challenge. Congruent with this, participants were engaged in motivational challenge or threat by requiring them to perform a demanding or non-demanding task prior to an information-processing task.

Our task was designed to simultaneously induce challenge and threat without compromising participants' capacity to process information during the subsequent task. Supporters of dual process models of information-processing (e.g. Eagly \& Chaiken, 1993; Petty \& Cacioppo, 1986; Petty \& Wegener, 1998) have demonstrated that the depletion of cognitive resources, for example, due to cognitive distractions or cognitive fatigue reduce the probability of cognitive elaboration. Based on this reasoning, a task was created that was not extremely demanding to perform - a visual ability game in which participants performed either a difficult or easy version long enough to promote task engagement and induce the correspondent challenge and threat states.

To observe the impact of challenge on information-processing, a strong or weak persuasion message was presented immediately after the motivational patterns were induced. It was expected that task engagement would carry on to the persuasion task and that challenged participants would not differentiate strong from weak arguments in attitudechange, suggesting less processing in this condition.

\section{Method}

\section{Participants and Design}

Fifty-two UCSB undergraduates (31 males; Mean age $=19.2 ; S D=1.22$ ) received course credit for their participation. Participants were randomly assigned to a 2 (demanding $v s$. non-demanding game) x 2 (strong $v s$. weak arguments) between subjects factorial design.

\section{Materials}

The material for this experiment consisted of a collection of 30 abstract geometrical shapes including triangles, squares, circles and rectangles created using the Microsoft Powerpoint program (see appendix E for material); a strong and weak persuasive message arguing against imposing restrictions on industry to minimize the effects of acid rain on the North Eastern states of the USA (the same message used in experiment 1); and mylar tape, 
electrodes and ZKG tape necessary to attach participants to the electronic equipment and obtain the physiological recordings.

\section{Procedure}

Participants arrived alone at the laboratory. First, they were greeted and given a consent form explaining the goals and procedures involved in the study. After they signed the consent form, the experimenter gave them an initial questionnaire (similar to the one used in experiment 1 that included demographic questions (gender, class, age) and atitudinal issues among which was our key statement ("The government should impose controls on industry to help minimize the effect of acid rain in the US") (see appendix B for complete questionnaire) that served as the pre-attitude measure. As in experiment 1, participants used the feeling thermometer to express how "cold" or "hot" they felt about the topic. Cold temperatures (from 0 to 49 ) reflected greater disagreement whereas hot temperatures reflected greater agreement (from 51 to 100).

After participants completed the questionnaire, the experimenter attached appropriate physiological sensors on participants' torsos and necks. Then, she asked them to sit in a comfortable chair and rest quietly so that baseline recordings could be collected. Participants were left alone in the room for a 5-minute period. Physiological recording were obtained using the same procedures and equipments of the previous experiment.

After the 5 minutes, the experimenter returned, stopped recording the physiological data, and gave the general instructions. Participants were informed that the goal of the study was to investigate individuals' visual abilities on different tasks while monitored physiologically. Participants were told that they would perform a visual-game task and a reading task. Furthermore, they were informed that all the instructions necessary to perform each task would be presented at the appropriate time via the computer. They were told to read the instructions carefully before starting each task. Next, the experimenter initiated the Medialab software on a PC-type computer that randomly assigned participants to the experimental condition and restarted the recording of the physiological responses. The experimenter was seated in the opposite side of the room for the entire session. Physiological recordings were made throughout the experiment.

For the first task, the instructions informed all participants that they were going to perform a visual ability game. The goal was to observe, on the computer screen, several pairs of geometrical shapes of different sizes, compare their length and then decide which 
one of the two was bigger. To increase task engagement, participants were informed that they only had 3 seconds to make each of their decisions and that most people could respond correctly within that interval. In addition, instructions informed participants that there were different types of trials and that the computer would prompt them with the type of the upcoming trial. Instructions were as follows: "After each trial and before the beginning of a new one, the computer will rapidly prompt you with the word "next". When you see the word "next", please focus and get ready because a new standard trial will start soon". Furthermore, participants in the non-demanding condition read the following on the computer monitor: "Note that, sometimes, the computer will randomly and rapidly prompt you with the word "non-demanding". This means that following this word you will see a different trial that maybe easier than the standard trial (i.e. when you see the prompt "next"). Thus, you have to pay attention to which word will pop up on the screen and be prepared to overcome every trial. Participants in the non-demanding condition read: "Note that, sometimes, the computer will randomly and rapidly prompt you with the word "demanding". This means that following this word you will see a different trial that may be harder than the standard trial (i.e. when you see the prompt "next") Thus, you have to pay attention to which word will pop up on the screen and be prepared to overcome every trial. Standard trials were added so that the demanding version would not be extremely difficult and the non-demanding would not be extremely easy.

To respond, participants used the computer mouse. They were informed that if they thought the shape presented on the left was bigger, then they should click on a box with an L ( $\mathrm{L}$ for Left) that would appear on the screen (below the geometrical shapes). If they thought the shape on the right was bigger, then they should click on the box with an $\mathrm{R}$ ( $\mathrm{R}$ for Right).

Each participant performed 30 trials presented successively on different screens in random order. Those in the non-demanding condition performed 15 standard trials, where the difference between the two shapes was relatively easy to discriminate $(1 \mathrm{~cm})$ and $15 \mathrm{non}$ demanding trials, where the difference was quite obvious and even easier to discriminate $(1.5 \mathrm{~cm})$. Those in the demanding condition performed 15 standard trials and 15 demanding trials, where the difference was not obvious and difficult to discriminate $(0.5 \mathrm{~cm})$.

After participants completed the task, new instructions informed them that they were going to continue the game later phase but in the interim they would perform the reading task. The reading instructions appeared on the screen for 20 seconds (the pre-tested time to 
read the instructions). ${ }^{5}$ Next, a strong or weak version of the acid rain message was presented, followed by the presentation of three different items (same used in experiment 1, see appendix A) anchored in a 7-point rating scale to assess participants' attitude towards the position defended in the message.

New instructions informed participants to continue the game. All participants performed 15 more standard trials. By the end of these trails, they received a bogus feedback of their performance. They were told that their performance was between $80 \%$ and $100 \%$ accuracy. Furthermore, they were told that the experiment was over.

The experimenter, then, turned off the physiological recordings and removed the electrodes and tapes from participant's body. Finally, participants were handed a final questionnaire to assess relevant control measures like the effectiveness of the challenge and threat manipulation, challenge and threat evaluations, participants' mood and motivation (see appendix F for complete questionnaire) and, in the end, they were fully debriefed and thanked for their participation.

\section{Dependent Measures.}

Attitude-Change Measure. Attitude-changes regarding acid rain were assessed via three items anchored in a 7-point rating scale. The three items revealed a single-factor solution (explaining more than $64 \%$ of the total variance) and a Cronbach's $\alpha=.72$. Based on the factor analysis, a composite measure of post attitude-change was created from which the pre-attitude measure was subtracted. This provided the attitude-change index.

Challenge and Threat Indexes. Following the same procedures in experiment 1, the mean values of $\mathrm{HR}, \mathrm{VC}, \mathrm{CO}$ and TPR were calculated for every minute of the baseline and every minute of both tasks. Subsequently, reactivity scores were calculated by subtracting $\mathrm{CV}$ responses on the $5^{\text {th }}$ minute of the baseline from the $1^{\text {st }}$ minute of the game task; the first minute of the persuasion task; and the minute in which participants rated their opinions. This provided three different reactivity indexes.

\footnotetext{
${ }^{5}$ We did this because we wanted to assure that individuals would still be task-engaged in the second task and, consequently, challenge and threat states would carry on to the information-processing task.
} 


\section{Results and Discussion}

\section{Challenge and Threat Data.}

Effectiveness of the Task-Demands Manipulation. The effectiveness of the manipulation of task-demands on the challenge and threat evaluations was confirmed when participant data from the item "In general, I think I had sufficient capacity to deal with what was demanded by the game" in a one-way ANOVA and a main effect of task-demands emerged (even though marginal), $F(1,48)=3.79 ; p=.06 ; \eta^{2}=.08$. Congruent with a cognitive evaluation of challenge, participants in the non-demanding condition reported more capacity to deal with the task-demands $(M=5.64 ; S D=1.62)$ than participants in the demanding condition $(M=4.76 ; S D=1.56)$ (see appendix $\mathrm{G})$.

Baseline Differences. When the $5^{\text {th }}$ minute of HR, VC, CO and TPR baseline values were entered as dependent measures in a 2 (demanding vs. non-demanding game) x 2 (strong vs. weak message) MANOVA, no differences were found regarding the impact of the demanding version of the game, $F(4,39)=1.60 ; p=.19$ or the message quality, $F<1$. Similarly, the interaction was not significant, $F(4,39)=2.02 ; p=.11$. These results suggest that baseline values were similar across our experimental conditions (see appendix $G$ ).

Task engagement. Game task. The hypothesis that participants were task-engaged during the performance of the game was confirmed via independent t-tests on both HR and $\mathrm{VC}$ reactivity indexes for this phase and significant effects emerged, showing that $\mathrm{HR}$ and VC were significantly different than $0, \mathrm{HR}, t(51)=3.23 ; p=.002 ; \eta^{2}=.17(M=12.77 ; S D=$ 28.52); VC, $t(51)=2.56 ; p=.01 ; \eta^{2}=.11(M=1.87 ; S D=5.28)$ (see appendix $\left.\mathrm{G}\right)$.

Task engagement. Persuasion task. Because the time between the game task and the persuasion task was short, it was expected that participants would remain task-engaged (and, consequently, engaged in the correspondent motivational state) in the persuasion task. This hypothesis was confirmed by the fact that HR and VC reactivity indexes for this phase significantly differed from $0, \mathrm{HR}, t(51)=3.82 ; p<.001 ; \eta^{2}=.22 \quad(M=12.86 ; S D=24.25)$; $\mathrm{VC}, t(51)=1.98 ; p=.05 ; \eta^{2}=.07(M=1.19 ; S D=4.36)$ (see appendix $\left.\mathrm{G}\right)$. 
Challenge and Threat indexes. Game task. The hypothesis that the non-demanding version of the game increased the challenge pattern of responses (corroborating the effectiveness of our manipulation) was tested by entering the CO and TPR reactivity indexes in two separate one-way ANOVA's with the game version as fixed factor. The main effect of game version emerged on both measures, $\operatorname{CO} F(1,50)=4.29 ; p=.04 ; \eta^{2}=.10 ; T P R F$ $(1,50)=5.47 ; p=.02 ; \eta^{2}=.12$. As expected, participants in the non-demanding game condition, in comparison with those in the demanding condition, exhibited a challenge pattern mapped by increases in $\mathrm{CO}$ (Mnon-demanding $=.62, S D=.48$ vs. Mdemanding = $.79, S D=.49$ ) and decreases in TPR (Mnon-demanding $=-107.71, S D=51.14 v s$. Mdemanding $=61.46, S D=25.60)($ see appendix $G)$.

Challenge and Threat indexes. Persuasion Task. Because participants remained task-engaged during the persuasion task, it was expected that those who performed the nondemanding version of the game would still be challenged during the persuasion task. This hypothesis was confirmed when TPR and CO reactivity indexes were entered in a 2 (demanding vs. non-demanding game) x 2 (strong vs. weak message) ANOVA. A main effect of game version emerged for both measures, $\operatorname{CO}, F(1,48)=4.36 ; p=.04 ; \eta^{2}=.10$; and TPR, $F(1,48)=3.93 ; p=.05 ; \eta^{2}=.09$. Congruent with the previously observed pattern, those who performed the non-demanding version were still more challenged while processing the persuasive message, than those who performed the demanding game (CO, Mnon-demanding $=1.22 ; S D=0.88$ vs. $\mathrm{CO}$, Mdemanding $=-1.48 ; \mathrm{SD}=0.92 ; \mathrm{TPR}$, Mnon-demanding = $92.30, S D=65.75$ vs. Mdemanding $=92.01, S D=61.48)^{6}$ (see appendix G).

\section{Attitude-Change Data.}

The general hypothesis associated with the impact of challenge and threat on depth of processing was that the engagement in a motivational state of challenge would decrease the probability of analytic processing. As such, it was predicted that those who were challenged (i.e. those who performed the non-demanding game before) would exhibit attitude-changes that did not reflect differentiation between strong and weak arguments. This hypothesis was

\footnotetext{
${ }^{6}$ Even though the message quality did not have any effects on $\mathrm{CO}\left(\mathrm{F}^{\prime} \mathrm{s}<1\right)$, it qualified the effect of the game version on TPR, $F(1,48)=4.91 ; p=.03 ; \eta^{2}=.17$. Whereas for those in the demanding condition the strong message, in comparison with the weak one, increased TPR values $(M=184.27 ; S D=89.33$ vs. $M$ weak $=-.25 ; S D=96.49)$, for those in the nondemanding condition it decrease them $(M=-206.15 ; S D=96.49$ vs. $M$ weak $=21.54 ; S D=89.33)$. The main effect of game version on TPR was not significant, $F<1$.
} 
supported when the acid-rain attitude-change index as was entered as a dependent measure in a 2 (demanding vs. non-demanding game) x 2 (strong $v s$. weak message) ANOVA. The predicted interaction emerged, $F(1,48)=4.11 ; p=.05 ; \eta^{2}=.08$. As hypothesized, this interaction was driven by the differential impact of strong and weak arguments on attitudechange manifested in the demanding (threat) condition, but not in the non-demanding (challenge) condition (see figure 3). Contrast analysis demonstrated that whereas those who were threatened seemed to be more persuaded by strong than by the weak message, $t(48)=$ $1.81 ; p=.07 ; \eta^{2}=.04$, those who were challenged reacted equally to strong and weak arguments, $t(48)=-1.06 ; p=.29 ; \eta^{2}=.02$. No main effect of the version of the game or message quality were found $\left(F^{\prime} s<1\right)$ (see appendix $\left.\mathrm{G}\right)$.

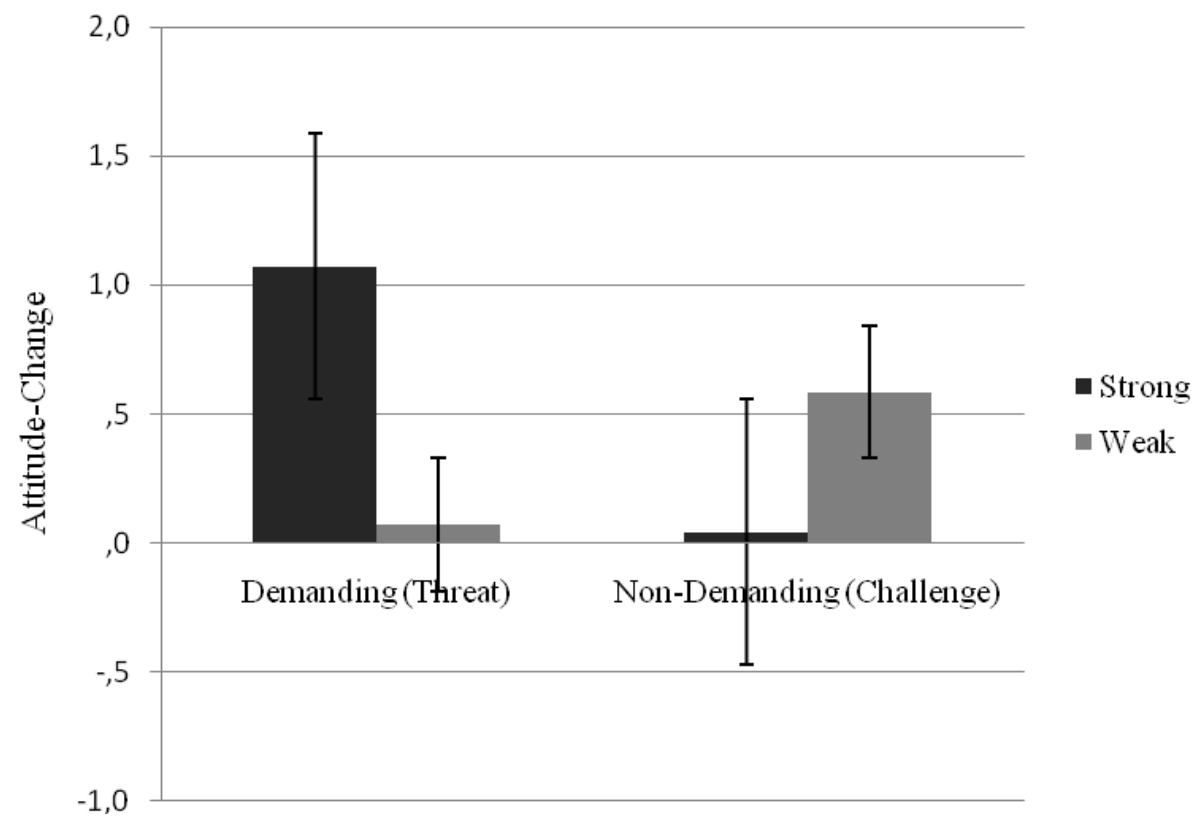

Figure 3. Attitude-change index towards the target issue as a function of demands and message quality.

These results provide the first evidence that conditions of increased challenge promote superficial processing. Congruent with the idea that familiarity and challenge are closely related, engagement in a motivational state of challenge reduces the differentiation between strong and weak arguments in attitude-change. As such, challenge seems to mimic effect of familiarity on information-processing depth. Additional relevant information offered by this study is that the type of engagement necessary to promote a motivational 
state of challenge or threat does not necessarily lead all individual to process information analytically. This may be either because it represented a moderate level of motivation or because the two constructs do not overlap.

These results were further explored by testing whether the cardiovascular markers, which map the motivational state, mediated the effect of challenge on informationprocessing. To do so, the unitary index of challenge and threat was calculated (using the $\mathrm{CO}$ and TPR reactivity indexes during the persuasion task) and entered as covariate in the previous 2 (demanding vs. non-demanding game) x 2 (strong vs. weak message) ANOVA model with the acid-rain attitude-change index as dependent measure. The possibility of mediation was ruled out when it was observed that the covariate had no impact on the dependent measure, $F<1^{7}$ (see appendix G).

Because evidence that participants in the non-demanding condition were also challenged is also supported by participants' cognitive evaluations of resources and demands, the possibility of mediation via these cognitive evaluations was also tested. However, when participants' responses on the item "In general, I think I had sufficient capacity to deal with what was demanded by the game" was entered as a covariate in the previous ANOVA model, the possibility of mediation was rejected as the covariate had no impact on the dependent measure, $F<1$ (see appendix G).

Additionally, because challenge is generally positive state, the possibility of mood mediation was also tested by averaging the three items assessing mood (How do you feel right now? 1 - Sad; 7 - Happy; How do you feel right now? 1 - Discontent; 7 - Content; How would you describe your mood at this time right now? $1-$ Bad; $7-$ Good) and entering the single measure (explaining more than $84 \%$ of the total variance; Cronbach's $\alpha=.89$ ) as covariate in the ANOVA model. Results showed that mood did not have a main effect on the measure rejecting therefore the possibility mediation, $F<1$ (see appendix $G$ ).

From these data, it can be concluded that the manipulation of the motivational state replicated the manipulation of familiarity shown in experiment 1 . Challenge reduced the differentiation between strong and weak arguments in attitude-change, like familiarity does, which is an indicator of more superficial processing. Evidence that participants in the challenge condition were indeed engaged in a motivational state of challenge is supported by

\footnotetext{
${ }^{7}$ Additional analysis also revealed that the impact of challenge and threat on information-processing is not mediated by TRP and CO raw values since both covariates had no impact on the dependent measure $F^{\prime} s<1$.
} 
the cardiovascular indexes and by the cognitive evaluations of challenge. However, surprisingly, as in Experiment 1 none of these measures mediated the challenge effects on how information was processed.

The reason why the responses that map the motivational state did not mediate the effects of challenge on processing is unclear. Unlike experiment 1, one cannot argue that our participants were not task-engaged during the time that measures were assessed. Challenge and threat patterns were assessed prior to the information-processing and were demonstrated that these patterns were continued after the game task to the moment in which participants were actually processing persuasive arguments. Also, unlike experiment 1 , it cannot be argued that the manipulation of task-demands did not engage participants in the correspondent motivational state at the moment of message processing because it both promoted the cardiovascular responses and the cognitive evaluations consistent with the state at that moment.

Because it was shown that familiarity and challenge manipulation have parallel effects, the hypothesis that there is a common factor underlying them seems plausible. The suspicion here is that the common factor is a signal of resource availability. But one must ask why this common factor did not emerge in either experiment 1 or 2. Clearly, both effects are only proxies and incomplete measures of the variable that is assumed to promote them. It is possible that the state of challenge may signal the availability of resources that impact processing because one has resources available or because the demands are low.

Together experiments 1 and 2 allow one to say that: a) familiarity is associated with a state of challenge; and b) a state of challenge promotes parallel effects of familiarity. Together, these effects along with possible criticisms of the "measurement" indexes make still possible the proposition that familiarity effects on processing are associated with that impact because they engage individuals in a challenge state. So a question that needs to be addressed further is whether the FARM effects imply that individuals are engaged in a challenge motivational state in familiar contexts.

From research on the BPS model, one can conclude that challenge effects are dependent upon task engagement. From the FARM assumptions, the impact of familiarity is most noticed under moderated motivation. The results of Experiment 2 made clear that individuals may be engaged in a motivational state of challenge and threat without necessarily make them more prone to process the content of a persuasive message analytically. So familiarity effects are expected to be noticed under task engagement 
conditions. The question is if no task engagement, which disrupts motivational states, also disrupts the familiarity effects. If that is the case, familiarity effects could be dependent upon the activation of a challenge state.

A factor known to influence task engagement is the presence (vs. absence) of others (Blascovich et al., 1999). It was expected that such manipulation of task engagement to promote dissociation in the familiarity and challenge effects. Because task engagement is necessary for the challenge effects, the impact of challenge on processing should be more strongly observed in the presence of an observer than in an alone condition. In opposition, because task engagement is not necessary for the familiarity effects, it should be expected that the effects of familiarity on processing are observed both in the presence and in the absence of an observer. Our next set of experiments tested this dissociation hypothesis by replicating experiment 1 and 2 with or without a presence of other manipulation.

The FARM and the BSP models assume familiarity or/and challenge as subjective experiences that inform our cognitive system that there is an available and accessible response in memory to perform the task. If the presence of other has indeed this dissociative effect on familiarity and on challenge effects it suggests that the mechanisms through which they occur are independent.

\section{Experiment 3}

The role of the presence of others on the effects of challenge and threat and familiarity on information-processing.

In this experiment, the hypothesis that the effect of challenge on informationprocessing is only exhibited when task engagement is activated as Blascovich et al. (e.g., Blascovich, 2008) maintain was tested. To do so, the presence or absence of an observer was manipulated, a variable should at least increase the intensity of task engagement. Because task engagement is necessary for the effects of challenge, it was expected that when task engagement is intensified (i.e. in the presence of the observer) challenge will decrease analytic processing but this effect should not occur when task engagement is not intensified (i.e. in an alone situation). 
To test these hypotheses, the same paradigm was used as in experiment 2 with the added manipulation of presence vs. absence of an observer. As in experiment 2, challenge and threat were induced via perception of task-demands. Specifically challenge and threat were induced by participants being accompanied or not by an observer while performing either a non-demanding or a demanding version of the visual ability task. Immediately after inducing the motivational states a strong or weak persuasive message was presented in order to allow us to test the predicted effects.

\section{Method}

\section{Participants and design}

Ninety-eight UCSB undergraduate students (55 females; Mage $=19.18 ; S D=1.32$ ) received course credit for their participation. Participants were randomly assigned to a 2 (demanding $v s$. non-demanding game) x 2 (strong $v s$. weak message) x 2 (alone vs. presence of observer) between subjects factorial design.

\section{Material}

The materials included the collection of abstract geometrical shapes used in experiment 2 (see appendix E); the strong and weak version of the acid rain message and the physiological sensors and equipment used in the previous experiments (see appendix A).

\section{Procedure}

This experiment was a close replication of experiment 2. First, participants arrived, were greeted and signed the consent form. Then, they were handed the initial questionnaire with demographic questions and used the feeling thermometer to express their attitudes towards several atitudinal issues, including one about acid rain which served as the preattitude measure.

Next, the appropriate sensors and transducers were attached to participants' and a 5 minute baseline of $\mathrm{CV}$ responses commenced. At the end of this period, the experimenter returned and stopped the recording of the physiological data. For those in the alone condition, the experimenter returned alone. For those in the presence condition, the experimenter returned accompanied by a male or female confederate (matching participant's gender) who sat in the room for the entire experiment. Similar to Blascovich et. al.'s 
investigation (1999), the confederate was introduced as follows: "This is a volunteer from our laboratory who is interested in how people perform tasks on a computer while being connected to physiological equipment. For this reason, he/she will be staying in the room throughout the experiment to observe your performance.

Then, participants were informed about the general goals of the experiment and were told that all instructions to perform each task would be presented on the computer screen. Before they started, the experimenter reinitiated the physiological recordings and then left the room.

For their first task, participants performed the visual ability game. As in experiment 2, instructions informed participants that they had 3 seconds to compare the sizes of two shapes and decide which one was bigger by clicking the mouse on the correct response box. Furthermore, instructions stressed the importance to be as fast and accurate as possible. All participants performed 30 trials presented in random order. They were informed that there were different types of trials. Before the presentation of each trial, the computer prompted participants with a word that hinted the type of trial they were about to encounter. The prompts were the word "next" (signalling the standard trials, i.e., those in which the difference between the shape was relatively obvious: $1 \mathrm{~cm}$ ); "non-demanding" (signalling the easy trials, i.e., those in which the difference between the shape was obvious: $1,5 \mathrm{~cm}$ ); or "demanding" (signalling the difficult trials, i.e., those in which the difference between the shape was not obvious: $0,5 \mathrm{~cm}$ ). Participants assigned to the challenge condition solved 15 standard trials and 15 non-demanding trials, whereas, those assigned to the threat condition solved 15 standard trials plus 15 demanding trials.

After the game was completed, the instruction informed participants that a new task was going to start right. For this task, participants were told to read the message that would appear on their computer screen carefully. The message was a randomly assigned strong or weak version of the acid-rain message. After reading it, participants rated their opinion towards the topic in three items anchored in a 7-point rating scale.

Next, the game was reinitiated for more 15 standard trials. Participants received bogus feedback on their performance; and were told that their participation was over. The sensors were then removed from participants. They were then asked to fill out the control measures questionnaire (see appendix F). Upon completion, participants were thoroughly debriefed and thanked for their participation. 


\section{Dependent Measures}

Attitude-Change Measure. Post-attitude measure of the acid rain revealed a singlefactor solution (explaining more than $80 \%$ of the total variance) and a Cronbach's $\alpha=.87$. This composite measure was then decreased by the pre-attitude measure providing an attitude-change index.

Challenge and Threat Indexes. Mean values of $\mathrm{HR}, \mathrm{VC}, \mathrm{CO}$ and TPR were calculated for every minute of the baseline; every minute of the game task and the persuasion task. Then, reactivity scores for each $\mathrm{CV}$ response were calculated by subtracting the $5^{\text {th }}$ minute of the baseline by the $1^{\text {st }}$ minute of the game task; and by the first minute of the persuasion task.

\section{Results and Discussion}

\section{Challenge and Threat Data.}

Effectiveness of the Task-Demands Manipulation. The effectiveness of the manipulation of task-demands on the challenge and threat evaluations was corroborated when by entering the item "In general, I think I had sufficient capacity to deal with what was demanded by the game" in a 2 (demanding $v$ s. non-demanding game) x 2 (alone $v s$. presence of observer) ANOVA and a main effect of task-demands emerged, $F(1,91)=16.12 ; p=.00$; $\eta^{2}=.21$. Consistent with cognitive evaluations of challenge, participants in the nondemanding condition reported more capacity to deal with the task-demands $(M=6.13 ; S D=$ $.21)$, than participants in the demanding condition $(M=4.97 ; S D=.20)$. No other main effects or interaction were found, $F^{\prime} s<1$ (see appendix $\mathrm{H}$ ).

Baseline Differences. The introduction of the $5^{\text {th }}$ minute of $\mathrm{HR}, \mathrm{VC}, \mathrm{CO}$ and TPR baseline values in a 2 (demanding vs. non-demanding game) x 2 (strong $v s$. weak message) $\mathrm{x}$ 2 (alone vs. presence of observer) MANOVA revealed no differences across our experimental conditions in $\mathrm{HR}, \mathrm{VC}$ and $\mathrm{TPR}, F^{\prime} s<1$. However, the results of the univariate analysis revealed that $\mathrm{CO}$ was significantly different in the presence condition, $F(1,90)=$ 
$4.55 ; p=.04 ; \eta^{2}=.10$, because alone participants exhibited greater levels of CO $(M=5.65$; $S D=.29)$ than accompanied participants $(M=4.78 ; S D=.29)$ even though there were no difference at that point in time in experimental protocol. In order to control for these differences, $\mathrm{CO}$ baseline values were entered as a covariate in the subsequent analyses (see appendix H).

Task engagement. Game task. To test the hypothesis that the presence of the observer increases the intensity of task engagement, it was expected that $\mathrm{HR}$ and $\mathrm{VC}$ reactivity scores for the game task to be different from 0 only in the presence condition. Results corroborate this hypothesis by showing that HR and VC were significantly different from 0 in the presence condition, HR, $t(49)=3.16 ; p=.003 ; \eta^{2}=.17 \quad(M=10.24 ; S D=$ 22.89); VC $t(49)=2.17 ; p=.06 ; \eta^{2}=.09(M=6.52 ; S D=23.87)$, but not in the alone condition, HR, $t(47)=1.93 ; p=.06 ; \eta^{2}=.08(M=5.06 ; S D=18.15)$; VC $t<1$ Because task engagement is a necessary condition for the activation of challenge and threat, challenge and threat indexes could not be computed for the alone condition (see appendix $\mathrm{H}$ ).

Task engagement. Persuasion task. Since the time between the two tasks was so short, it was expected that participants in the presence condition would be task-engaged during the persuasion task. This hypothesis was confirmed by the fact that HR and VC reactivity indexes for this phase were significantly different from $0, \mathrm{HR}, t(49)=2.35 ; p=$ $.02 ; \eta^{2}=.12(M=5.92 ; S D=17.87) ; \mathrm{VC}, t(49)=1.93 ; p=.04 ; \eta^{2}=.04(M=7.33 ; S D=$ $24.15)^{8}$.Again, because task engagement is a necessary condition for the activation of challenge and threat, these indexes could not be computed for the alone (see appendix $\mathrm{H}$ ).

Challenge and Threat indexes. Game task. In support of the hypothesis that the non-demanding version of the game induced a pattern of challenge, a main effect of the game version was found on both $\mathrm{CO}$ and TPR when both measures were entered as dependent measures in separate one-way ANOVAs, CO, $F(1,47)=3.65 ; p=.06 ; \eta^{2}=.07$; TPR, $F(1,47)=5.03 ; p=.03 ; \eta^{2}=.10$. Consistent with a challenge pattern, the nondemanding version, compared with the demanding version, promoted greater increases in $\mathrm{CO}$ (Mnon-demanding $=.92, S D=0.44 v s$ Mdemanding $=-.26, S D=0.42)$ and decreases in

\footnotetext{
${ }^{8}$ We also analyzed the $\mathrm{HR}$ and $\mathrm{VC}$ values for the alone condition to test if these individuals were engaged in this task but the results rule out this hypothesis, HR, $t(47)=1.95 ; p=.06 ; \eta^{2}=.08(M=4.94 ; S D=17.54) ; \mathrm{VC}, t<1$.
} 
TPR $(\text { Mnon-demanding }=-331.194, S D=165.47 \text { vs. } \text { Mdemanding }=193.45, S D=158.72)^{9}$. These results corroborated the ones found in experiment 2 and demonstrated the success of our manipulation in inducing the challenge and threat states (see appendix $\mathrm{H}$ ).

Challenge and Threat indexes. Persuasion Task. Because task engagement was active during the persuasion task, it was expected that those who performed the nondemanding version of the game would remain challenged during this task. This hypothesis was confirmed when $\mathrm{CO}$ and TPR reactivity indexes were entered in separate 2 (demanding vs. non-demanding game) $\mathrm{x} 2$ (strong $v$. weak message) ANOVAs and a main effect of the game version emerged for both measures, $\mathrm{CO}, F(1,46)=3.87 ; p=.06 ; \eta^{2}=.08$; TPR, $F$ $(1,46)=4.41 ; p=.04 ; \eta^{2}=.09^{10}$. Congruent with a challenge pattern, those in the nondemanding condition, compared with those in the demanding version, exhibited greater increases in $\mathrm{CO}$ (Mnon-demanding $=1.62, S D=.53 \mathrm{vs}$. Mdemanding $=.13, S D=.51)$ and decreases in TPR (Mnon-demanding $=-90.29, S D=195.22$ vs. Mdemanding $=489.08, S D=$ 185.52).

The quality of the message did not have a direct impact on CO, $F(1,46)=1.48 ; p=$ .23 , and it did not interact with the demands factor, $F(1,46)=1.06 ; p=.31$. Similarly, no main effect, $F(1,46)=1.66 ; p=.20$ no interaction, $F<1$ with this factor was found for TPR (see appendix $\mathrm{H}$ ).

Corroborating the findings in experiment 2, the manipulation of challenge and threat carried over from the game task to the moment participants began processing the persuasive message.

\section{Attitude-Change Data.}

Because task engagement is necessary for the effects of challenge, it was hypothesized that challenge would decrease analytic processing in the presence condition (where task engagement was active), but not in the alone condition. In other words, it was expected that the challenge effects observed in experiment 2 would only be evident in the presence condition.

This hypothesis was confirmed when the acid-rain attitude-change index was entered in a 2 (demanding vs. non-demanding game) x 2 (strong vs. weak message) x 2 (alone $v s$.

\footnotetext{
${ }^{9}$ The covariate (CO baseline) did not have a direct impact on CO or TPR, $F<1$.

${ }^{10}$ The covariate (CO baseline) did not have a direct impact on CO or TPR, $F<1$.
} 
presence of observer) ANOVA resulting in a marginally significant demands $\mathrm{x}$ presence interaction, $F(1,90)=3.19 ; p=.08 ; \eta^{2}=.06$, moderated by the presence factor, $F(1,90)=$ $4.87 ; p=.03 ; \eta^{2}=.05$. When the data were analyzed separately by the presence condition, it was observed that the demands $\mathrm{x}$ message quality interaction occurred in the presence of others condition, $F(1,46)=6.51 ; p=.01 ; \eta^{2}=.22$, but not in the alone condition, $\mathrm{F}<1$. As predicted, in the presence of other condition, the differential impact of strong and weak arguments on attitude-change was manifested in the demanding (threat) condition but not in the non-demanding (challenge) condition (see figure 4). Contrast analysis demonstrated that whereas threatened participants were more persuaded by strong than by the weak arguments, $t(26)=2.04 ; p=.04 ; \eta^{2}=.10$, those who were challenged did not differentiate the argument quality in attitude-change, $t(24)=1.58 ; p=.12 ; \eta^{2}=.03$.

No main effects of message quality or game demands were found in the alone condition, $F^{\prime} s<1$ (see appendix $\mathrm{H}$ ).

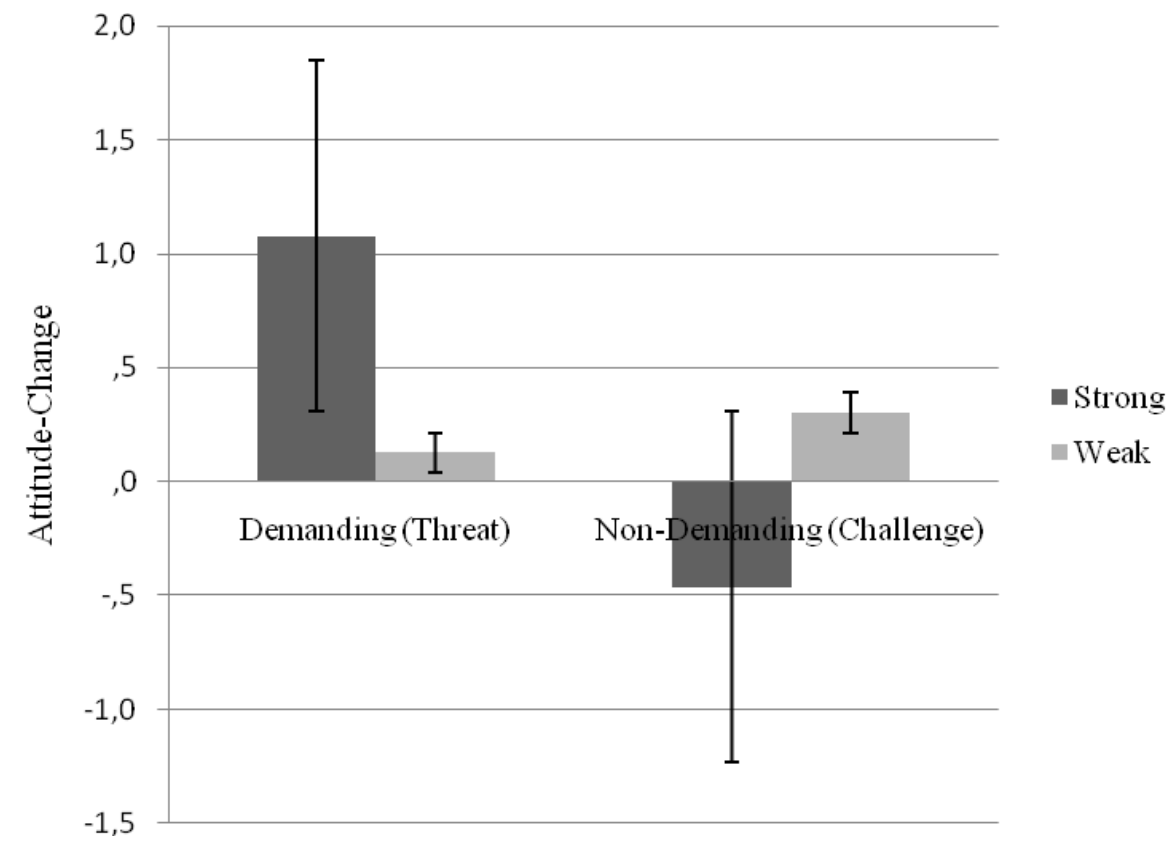

Figure 4. Attitude-change index towards the target issue as a function of motivational state and argument strength for the presence condition.

The fact that no effect was observed in the alone condition is relevant to our argument. Although no effect of the motivational state was expected in the alone condition 
because of the lack of task engagement, it could be that participants reacted differently to strong and weak arguments. The fact that they did not suggests that they were not elaborating the message content. In some way, the lack of task engagement produces superficial processing, suggesting a low level of motivation.

These results replicate the effect observed in experiment 2 supporting the hypothesis that challenge decreases the probability of analytic processing. In addition, they make clear that the effect is prone to the activation of the motivational state, being present only when task engagement is increased. The effect seems to be dependent upon establishing the motivational states and not of any other cues offered by the manipulation procedures. These data suggest that without full task engagement and specific activation of different motivational states, the challenge effect on information-processing would not occur.

However, as in previous studies, the effect does not seem to be mediated by the cardiovascular responses. When the challenge and threat index was entered as a covariate, relative to the persuasion task minutes (ZTPR + ZCO) in the 2 (demanding vs. nondemanding game) x 2 (strong vs. weak message) x 2 (alone vs. presence of observer) ANOVA, covariate was not significant, $F<1$ (see appendix $\mathrm{H}$ ).

Contrary to previous studies, however, some evidence of a moderation effect on the measures of cognitive evaluations of challenge and threat was obtained. When the item "In general, I think I had sufficient capacity to deal with what was demanded by the game" was entered as a covariate in the model, results revealed that it has a significant impact on the dependent measure, $F(1,86)=3.99 ; p=.05$. However, even though it seems to explain some of the variance in the results, the previously found three-way interaction remained significant (even though marginal), $F(1,89)=3.01 ; p=.09$ (see appendix $\mathrm{H}$ ).

The detection of this effect of the covariate may in some way be related to the increased engagement promoted by the evaluation apprehension (e.g. Bond, 1982; Cottrel, Wack, Sekerak \& Ritlle, Guerin, 1983) that comes from being observed by others. Contrary to experiment 2 in experiment 3 , a confederate was explicitly presented as someone who was going to "observe participants' performance".

Mood does not seem to be mediating the effects as the introduction of the composite measure of the three items assessing it (59\% of total variance explained; Cronbach's $\alpha=.46$ ) as a covariate in the ANOVA model revealed no main effect of this variable on the dependent measure, $F>1$ (see appendix $\mathrm{H}$ ). 
So, based on these, it can be stated that challenge decreases the probability of analytic processing but only when task engagement is intensified. As expected, without task engagement this motivational state has no impact on information-processing.

No mediation effects of physiological indexes were found in this experiment.

\section{Experiment 4}

According to the FARM model, task engagement is not necessary to observe the familiarity effects. So, if familiarity is independent of task engagement, an equal impact of familiarity both in a condition of intensified task engagement (i.e. in a presence of an observer condition) and in a condition of decreased task engagement (i.e. in an alone condition) should be observed.

To test this hypothesis, experiment 1 was with the added condition of presence vs. absence of an observer. As in the previous experiment, the paradigm comprised a task that served the purpose of inducing familiarity. It was another persuasion task that tested the impact of familiarity on information-processing; specifically, a speech task in which the impact of familiarity on information-processing and motivational challenge and threat were examined.

It was expected that the previous finding that familiarity reduces the differentiation between strong and weak arguments in attitude-change would be replicated; that is, this effect should be manifested in both a presence and an isolation condition. However, because participants in the alone condition of experiment 3 did not react differently to strong and weak arguments, it is possible that an interaction emerges, suggesting that the effect is observed only in presence of others because of motivation to processing information deeply.

\section{Method}

\section{Participants and Design}

Eighty-three UCSB undergraduate students (41 females; Mage $=18.9 ; S D=1.29$ ) received course credit for participation. Participants were randomly assigned to a 2 (strong 
$v s$. weak message) x 2 (repetition $v s$. non-repetition situation) x 2 (alone vs. presence of a confederate) between subjects factorial design.

\section{Material.}

The same persuasive messages as in the previous experiments were utilized. There was a strong and weak version of a message arguing for the effectiveness of weight loss centers; a strong and weak version of a message arguing against restrictions on industry to minimize the effects of acid rain; and a strong and weak version of a message arguing for increases in road taxes (see appendix A). Moreover, the same equipment and procedures to collect the physiological data were employed.

\section{Procedure.}

The procedure used in experiment 1 was followed closely. Participants were greeted at their arrival at the laboratory and asked to read and sign the consent form. Next, they were handed the initial demographic questionnaire with additional attitudinal questions and regarding key statements about weight loss centers and acid rain (pre-attitude measures). Participants used the feeling thermometer (in the same way as in the previous experiments) to give their responses.

As before, the experimenter applied the physiological sensors and baseline recordings of the participants' $\mathrm{CV}$ responses were collected for 5 minutes. Next, the experimenter returned to the room and stopped recording the physiological data. For those in the alone condition, the experimenter came alone. For those in the presence condition, the experimenter came accompanied by a male or female confederate (matching participant's gender) who sat in the room for the entire experiment. The confederate was introduced the same way as in experiment 3 (a volunteer interested in how participants perform computer tasks while hooked-up to physiological equipment).

After introducing the confederate, the experimenter gave the general instructions about the experiment's goal (same used in experiment 1), set up the computer program, restarted the recording of the physiological data and sat away from both the confederate and the participant. Physiological recordings were taken throughout the experiment.

On the first computer screen, participants received instructions for the first task. They were told that the upcoming task involved doing simultaneous activities: reading a message on the computer screen, while listening to an unrelated background message. Instructions 
stressed that the first and most important task was the reading one and as such they should ignore the audio message. Then, participants put on the provided headphones and began reading a randomly assigned strong or weak message arguing for the effectiveness of the weight loss centers. For those in the no-repetition condition, the audio message was one arguing for an increase in road taxes. For those in the repetition condition, the audio message argued against imposing restrictions on industry to minimize the effects of acid rain. Both the written and audio messages were matched in their strength. Then, participants rated their opinions towards weight loss centers in three items (same used in experiment 1) anchored in a 7-point rating scale.

Next, new instructions informed participants that the ensuing task required them only to read carefully a message on the computer screen. Participants took off the headphones and started reading a randomly assigned strong or weak message arguing against imposing restrictions on industry to control the effects of acid rain. For those in the repetition condition, this was the same message played over the headphones in the previous task. For those in the no-repetition condition, this was their first exposure to this message. Then, participants rated their opinions towards the acid rain message in three items anchored in a 7-point rating scale.

For their last task, participants were instructed to recall the thoughts they had while reading the acid rain message. Next, they were asked to prepare a speech based on these thoughts and then deliver it to a video-camera. Participants had one minute to prepare the speech and three minutes to deliver it.

By the end of the speech task, the experiment was over. The experimenter turned off the physiological recording equipment and removed the electrodes and tapes from participant's body. Finally, participants filled out the control measures questionnaire (see appendix C); were fully debriefed and thanked for their participation.

\section{Dependent Measures.}

Attitude-Change Measure. Post-attitude measures of the weight loss centre revealed a single-factor solution for the three items used (explaining more than $72 \%$ of the total variance; Cronbach's $\alpha=.81$ ). Similarly, post-attitude measures of the acid rain revealed a single-factor solution (explaining more than $56 \%$ of the total variance) and a Cronbach's $\alpha=$ .60). Both post-attitude composite measures were subtracted by their correspondent preattitude measure to provide an attitude-change index for each message. 
Thought Measure. The number of thoughts generated by each participant and their favourability served as the measure of cognitive elaboration during the motivational taskperformance.

Challenge and Threat Indexes. Mean values of $\mathrm{HR}, \mathrm{VC}, \mathrm{CO}$ and $\mathrm{TPR}$ were calculated for each minute of the baseline and the speech task. Then, reactivity scores for each CV response were calculated by subtracting the $5^{\text {th }}$ minute of the baseline by the $1^{\text {st }}$ minute of the speech task.

\section{Results and Discussion}

\section{Attitude-Change Data.}

Effectiveness of the Manipulation of familiarity. The success of the familiarity manipulation was corroborated by a main effect of repetition that emerged when the item "The presentation of the audio message on phase 1 made the second written message more familiar", was entered in a 2 (repetition vs. no-repetition condition) x 2 (strong vs. weak message) x 2 (presence vs. absence of observer) ANOVA, $F(1,75)=12.33 ; p=.001$; $\eta^{2}=20$. As expected, participants in the repetition condition, considered the presentation of the message more familiar $(M=4.75 ; S D=0.27)$, than those in the no-repetition condition $(M=3.39 ; S D=0.28)$. Results also found a marginally significant main effect of the presence condition, $F(1,75)=3.20 ; p=.08 ; \eta^{2}=.06$, suggesting that those who were observed considered the message as more familiar $(M=4.42 ; S D=0.28)$, than those who were alone $(M=3.72 ; S D=0.27)$ (see appendix I).

The interaction between the presence and the familiarity factor was not significant, $F$ $<1$. Similarly, the quality of the message did not have a direct effect on the item, nor did it moderated the familiarity effect or the presence effect on the item, $F^{\prime} s<1$. Similarly, the three way interaction was not significant, $F(1,75)=2.35 ; p=.13$.

Attitude-Change towards the weight loss center message. Because the instructions stressed the importance of attending carefully to message content, it was expected that message quality effects such that participants would be more persuaded by the strong than 
by the weak message. The attitude-change index relative to this issue was entered in a 2 (strong vs. weak message) x 2 (repetition vs. non-repetition condition) x 2 (alone vs. presence of observer) ANOV ${ }^{11}$. Results suggested that as expected, participants agreed with the claim more after exposure to the strong version of the message $(M=1.49 ; S D=0.25)$, than exposure to the weak version $(M=0.97 ; S D=0.23)$, even though this effect did not reach significance, $F(1,56)=2.25 ; p=.14 ; \eta^{2}=.03$.

Neither a main effect of repetition, $F<1$ nor presence emerged, $F(1,56)=2.51 ; p=$ .12. Similarly, the effect of argument quality was not moderated by repetition, $F<1$ or by the presence of others, $F(1,56)=1.17 ; p=.28$. The three-way interaction was not significant, $F$ $<1$ (see appendix I).

The lack of interaction between repetition and argument quality suggests that all participants engaged (as expected) in more deep, analytic processing and the lack of a threeway interaction suggested that this was independent of the presence condition.

Attitude-Change towards the Acid-rain Message. In order to test the hypothesis that familiarity reduces the differentiation between strong and weak arguments in attitudechange independently of the presence condition, the attitude-change index was entered relative to this issue as a dependent measure in a 2 (repetition $v s$. no-repetition condition) $\mathrm{x} 2$ (strong vs. weak message) x 2 (alone vs. presence of observer) ANOVA. In support of this hypothesis, a significant repetition $\mathrm{x}$ message quality interaction emerged (although marginal), $F(1,75)=3.04 ; p=.09 ; \eta^{2}=.05$. As expected, this interaction was driven by a familiarity moderation of the differential impact of strong and weak arguments on attitudechange, manifested in the no-repetition condition, but not in the repetition condition. Contrast analysis suggested that whereas those who read the message for the first time were significantly more persuaded by the strong than by the weak message, $t(41)=2.60 ; p=.01$; $\eta^{2}=.23$, those in the repetition condition reacted equally to strong or weak arguments $t<1$ (see Figure 5). Also, as predicted this effect was not moderated by the presence factor, $F<1$. Thus, contrary to experiment 3, participants in the alone condition who did not feel the situation as familiar engaged in more deep processing.

\footnotetext{
${ }^{11}$ Due to a computer failure in saving participants' responses, we lost 17 responses on the three items measuring attitudes towards the weight loss center message. Thus our total sample for phase 1 was $\mathrm{N}=64$.
} 


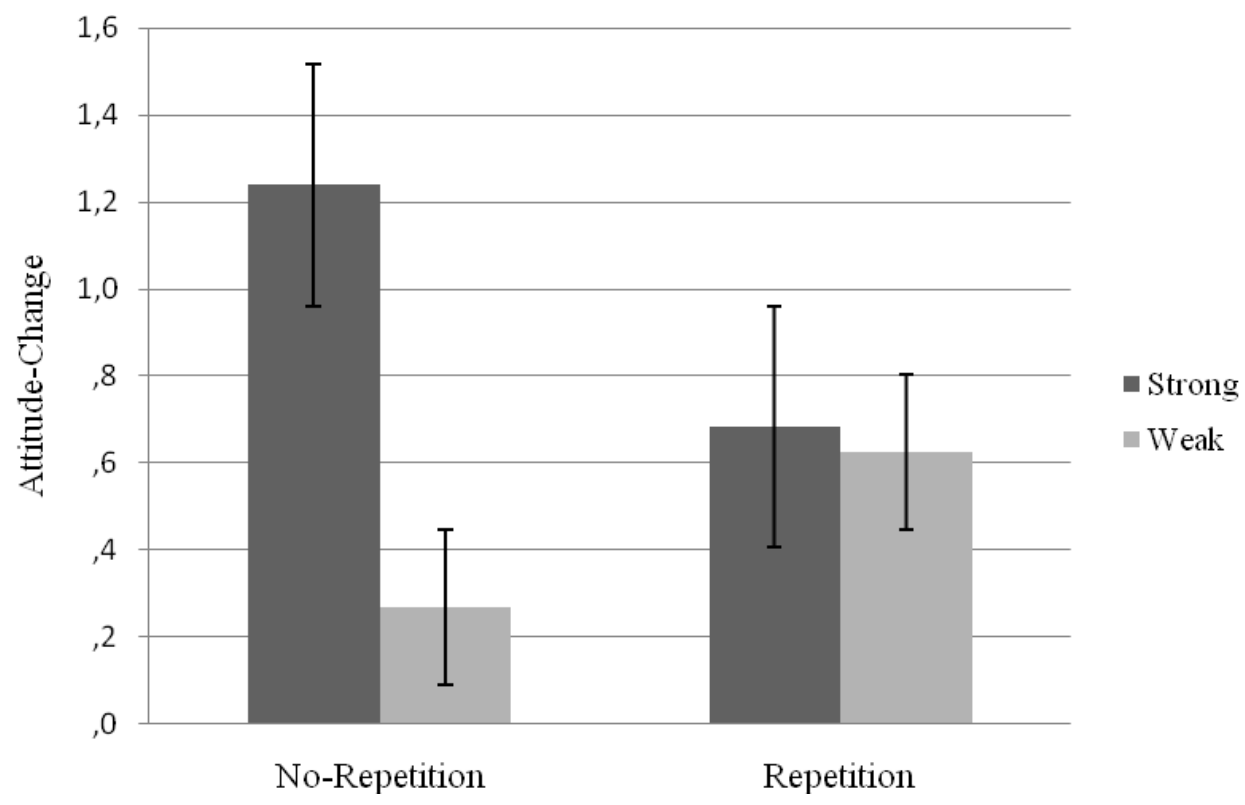

Figure 5. Attitude-change index towards the target issue as a function of message repetition and argument quality.

Results also revealed a main effect of message quality, $F(1,75)=3.88 ; p=.05$; $\eta^{2}=.09$, showing that the strong message promoted more attitude-changes than the weak (Mstrong= $.96 ; S D=.19$ vs. Mweak $=.45 ; S D=.18)$; and a significant presence $\mathrm{x}$ repetition interaction, $F(1,75)=10.51 ; p<.01 ; \eta^{2}=.13$, suggesting that whereas alone participants were more convinced by the new message $(M=1.19 ; S D=.25)$, than by the "old" $(M=.25$; $S D=.26)$, the observed participants were more convinced by the "old" message $(M=1.06$; $S D=.26)$, than by the new $(M=.31 ; S D=.27)$.

The presence factor did not interact with the message quality factor, $F(1,75)=1.35$; $p=.25$. No other effects were found, $F^{\prime} s<1$ (see appendix I).

These results replicate evidence from the FARM model and from experiment 1 and support the view that the presence of others does not moderate the impact of familiarity on information-processing.

\section{Thoughts Data.}

Following the analyses performed in experiment 1, the number of thoughts as a measure of elaboration was used. It was expected that repetition would decrease the number of thoughts generated, this effect being independent of the presence condition. The number 
of thoughts generated by each participant in a 2 (repetition $v s$. no-repetition condition) $\mathrm{x} 2$ (strong $v s$. weak message) x 2 (alone $v s$. presence of observer) was analyzed.

As expected, the main effect of repetition emerged, $F(1,64)=4.95 ; p=.03 ; \eta^{2}=.11$, showing that those in the repetition condition generated less thoughts $(M=1.99 ; S D=0.18)$, than those in the no-repetition condition $(M=2.57 ; S D=0.19)$. This effect was moderated by the presence factor, $F(1,64)=7.73 ; p<.01 ; \eta^{2}=.22$. This interaction seems to be explained by the fact that observed and alone participants generated different numbers of thoughts in the no-repetition condition (Mobserved $=3.08 ; S D=.28$ vs. Malone $=2.06 ; S D=$ $.25, t(41)=2.21 ; p=.03 \eta^{2}=.12$ ), but not in the repetition condition as predicted (Mobserved $=1.78 ; S D=.25$ vs. Malone $=2.20 ; S D=.26, t<1)^{12}$ (see appendix I).

Because only those in the no-repetition condition were expected to attend to the quality of the message, familiarity should qualify the impact of message quality on the favorability of thoughts. Three independent judges coded each thought into favorable, unfavorable or neutral categories in reference to the position defended by the message (Cohen's Kappa $=.83$ ) and then the proportion of favorable and unfavorable thoughts were analyzed as repeated measures in a 2 (favorable vs. unfavorable thoughts) x 2 (strong vs. weak arguments) x 2 (repetition vs. no-repetition) × 2 (alone vs. presence of observer) ANOVA. As predicted, the results suggested a significant type of thoughts $\mathrm{x}$ message quality interaction, $F(1,68)=5.76 ; p=.02 ; \eta^{2}=.12$ moderated by familiarity (although marginally), $F(1,68)=2.65 ; p=.11 ; \eta^{2}=.02$. As shown in table 2 , the number of favorable thoughts differed as a function of message quality in the no-repetition condition, $t(38)=2.83 ; p<.01$; $\eta^{2}=.24$, but not in the repetition condition, $t<1$. These effects were independent of the presence condition, $F<1$. No other main effects or interactions emerged, $F^{\prime}$ s $<1$ (see appendix I).

\begin{tabular}{ccccc}
\hline \multicolumn{4}{c}{ No-Repetition } & \multicolumn{2}{c}{ Repetition } \\
\hline Type of & Strong & Weak & Strong & Weak \\
Thought & & & & \\
\hline Favorable & $.47(.08)$ &, $15(.09)$ & $.24(.08)$ & $.30(.08)$ \\
Unfavorable & $.17(.09)$ & $.43(.10)$ & $.24(.09)$ & $.42(.09)$
\end{tabular}

Table 2. Proportion of favorable and unfavorable thoughts as a function of message repetition and message quality.

\footnotetext{
${ }^{12}$ Our general model also revealed a main effect of message quality, $F(1,62)=4.95 ; p=.03 ; \eta^{2}=.11$, suggesting that the strong message induced more thoughts $(M=2.57 ; S D=.18)$ than the weak $(M=1.99 ; S D=.19)$. No other effect was significant, $p^{\prime} s>13$ (see appendix I).
} 
As in experiment 1, whether the thoughts favorability associated with strong and weak messages mediated the impact of familiarity on processing was tested. Because individuals in the no-repetition condition were attending to the quality of the message (and had thoughts that reflected the quality of it), it was expected that the favorability of the thoughts would mediate the effects of argument quality on attitude-change (Greenwald, 1968). Congruent with the results above, it was expected that this effect would occur in both presence groups.

To test this mediation hypothesis, an index of thought favorability was created for each participant (Proportion Favorable - Proportion Unfavorable / N thoughts, Tormala, Briñol \& Petty, 2006). Next, it was entered as a predictor in a regression analysis model in which the acid-rain attitude change-index was the dependent variable and the message quality the predictor.

Results revealed that the thought favorability index did not mediate the impact of message quality in the no-repetition condition given that the impact of message quality on attitude-change $(b=.42, t(39)=2.90, p=.006)$ was still significant when the thought favorability index was controlled $(b=.54 ; t(26)=2.31, p=.03)$. This reduction not significant $($ Sobel $=.82 ; p=.41)$. This is most likely explained by the fact that, even though participants were having thoughts congruent with the message quality, the thought favorability index did not have a direct impact on attitude-change $t<1$ (see appendix I). The thought favorability index did not predict attitude-change in the repetition condition, nor did it mediate the effects of message quality on attitude-change in either of the presence groups $\left(t^{\prime} s<1\right)$.

Taken together these results corroborate the previous findings that familiarity decreases the probability of analytic processing (as shown by the equal impact of strong and weak arguments on attitude-change and the fewer thoughts generated in this condition). In addition, these effects make clear that, as the FARM assumed, the familiarity effects are independent of level of task engagement here manipulated as the presence of others.

\section{Challenge and Threat Data.}

Baseline Differences. No baseline differences across conditions were found when the $5^{\text {th }}$ minute of HR, VC, CO and TPR baseline values were entered as dependent measures in a 2 (repetition $v s$. no-repetition condition) x 2 (strong $v s$. weak message quality) x 2 (alone 
$v s$. presence of observer) MANOVA and no main effects or interactions emerged, $p^{\prime} s<.19$ (see appendix I).

Task engagement. Results of t-tests supported the hypothesis that the presence of the observer increased task engagement the HR and VC reactivity indexes for the speech task were tested against $0^{13}$. As expected, whereas those in the presence condition were taskengaged, HR, $t(49)=3.16 ; p=.003 ; \eta^{2}=.17(M=10.24 ; S D=22.89) ; \operatorname{VC} t(49)=2.17 ; p=$ $.04 ; \eta^{2}=.09(M=4.95 ; S D=16.09)$, those in the alone condition were not, HR, $t(42)=1.99$; $p=.06 ; \eta^{2}=.09(M=8.35 ; S D=27.48) ; \mathrm{VC}, t(42)=1.27 ; p=.21 ; \eta^{2}=.04(M=4.59 ; S D=$ 23.76). Recall that because task engagement is a necessary condition for the activation of challenge and threat, the challenge and threat indexes for the alone condition could not be calculated (see appendix I).

Challenge and Threat indexes. To test if familiarity increased the pattern of responses associated with challenge, as in experiment 1, CO and TPR reactivity indexes were entered for the speech task as dependent measures in two separate 2 (repetition vs. norepetition condition) x 2 (strong $v s$. weak message) ANOVAs. These analyses were run only for the presence condition because in the alone condition a different pattern of challenge and threat could not be detected, i.e., task engagement was not reached. Following the previous findings here, results revealed a main effect of familiarity for both $\mathrm{CO}, F(1,36)=4.81 ; p=$ $.04 ; \eta^{2}=.10$ and TPR, $F(1,36)=5.38 ; p=.03 ; \eta^{2}=.11$. Consistent with a challenge pattern, those in the repetition condition, compared with those in the no-repetition condition, exhibited greater increases in CO (Mrepetition $=1.97, S D=1.19$ vs. Mno-repetition $=-1.81$, $S D=1.25)$ and decreases in TPR (Mrepetition $=-261.25, S D=147.89$ vs. Mno-repetition $=$ 236.97, $S D=155.52$ ) (see appendix I).

To address the question of whether or not the familiarity effects on informationprocessing are related with individuals engaging in a challenge motivational state in familiar contexts, we tested if the cardiovascular markers that map the motivational state could possibly mediate the familiarity effects. A unitary index of challenge and threat was calculated for the speech task and entered into a 2 (strong vs. weak) x 2 (repetition vs. norepetition) ANOVA with the acid-rain attitude-change index as dependent measure. The

\footnotetext{
${ }^{13} \mathrm{We}$ also tested if participants were task-engaged during the persuasion task, but the results did support this hypothesis, $\mathrm{HR}$ and $\mathrm{VC} t^{\prime} s<1$.
} 
cardiovascular index did not seem to be moderating the effects of familiarity, as it does not have a significant main effect on the dependent measure, $F<1$. Also, whether there was a possible mediation via cognitive evaluations of resources and demands was tested (by entering the item "In general, I think I had sufficient capacity to deal with what was demanded by the task" as covariate in the previous model). The results revealed that even though the covariate had a marginally significant impact on the measure, $F(1,75)=3.99 ; p=$ .06 , the possibility of mediation is ruled out as the previously found repetition $\mathrm{x}$ message quality interaction remained unaffected, $F(1,75)=3.04 ; p=.09 ; \eta^{2}=.05$ (see appendix I).

The possibility of mediation of challenge by the effects of familiarity was also tested by entering the unitary index in a 2 (strong vs. weak) x 2 (repetition vs. no-repetition) ANOVA with the number of thoughts (measure of elaboration) as dependent measure. . Results rule out this hypothesis since the covariate was not significant, $F<1$. Similarly, when the cognitive evaluations were entered as covariate in the model results showed no main effect of this variable on the dependent measure, $F<1$ (see appendix I).

As in previous studies, mood does not seem to mediate these effects. The introduction of a composite measure of three items assessing mood (74\% of total explained variance; Cronbach's $\alpha=.82$ ) as covariate in the 2 (strong vs. weak) x 2 (repetition vs. norepetition) ANOVA model, with acid-rain attitude change index as dependent measure, revealed that it has no effect on the dependent measure, $F<1$. Similarly, when the number of thoughts was used as a dependent measure, mood had no impact as covariate on the dependent measure, $F<1$ (see appendix I).

From these data, it can be concluded that the effects assumed by the FARM occurred independently of task engagement since both observed and alone participants (who were not task-engaged) processed the repeated message less analytically than the non-repeated message. As in Experiment 1, it was observed that the familiarity condition activated a cardiovascular pattern of challenge. Finally, replicating all other studies, the effects of information processing are not explained by the cardiovascular response pattern activated by the situation.

Even if the meditational data justifying the claim that familiarity effects are independent of the activation of a motivational state of challenge, the independence is corroborated here by the occurrence of the effect independent of the level of engagement necessary for its establishment. Thus, although there is no doubt that familiarity is related to 
challenge, it is likely that it occurs because of the information the state and the feeling carry for the cognitive system, one is not reducible to the other. 
In this thesis, support for the general hypothesis that familiarity reduces the probability of analytic processing and activates a motivational state of challenge was found. The first experiment clearly demonstrates that those exposed to a familiar situation compared to those in a novel situation did not engage in deep processing of the content of a persuasive message (corroborating past research on the FARM model, Garcia-Marques, 1999; Garcia-Marques \& Mackie, 2001, 2007). The same individuals exhibited a cardiovascular pattern of responses consistent with motivational challenge (corroborating past research on the BPS model, Blascovich et al., 1993, 1999). Even though these effects occur simultaneously, they do not appear integrated in a common process as shown by the absence of mediation of the markers of the motivational state (i.e. cardiovascular responses, cognitive evaluations and participants' ratings of mood) on the cognitive effect. Thus, even though familiarity leads to both predicted effects, we find no evidence that the two effects are related directly.

However engagement in motivational challenge, by itself, mimics the effects of familiarity on information-processing. Data from the second experiment show that when challenge and threat states are induced prior to the information-processing task, the engagement in motivational challenge resulted in less analytic processing, compared with the engagement in threat motivation. But again, like in experiment one, the markers of the motivational state (i.e. the cardiovascular responses, cognitive evaluations and ratings of mood) did not mediate the cognitive effect.

Our subsequent studies show these two effects, familiarity and challenge impact on processing as dependent upon individuals' level of task engagement. By replicating experiment two in an isolated environment and so reducing task engagement, challenge effects were not observed. As the third experiment demonstrated, whereas the manipulation of challenge led to the correspondent cardiovascular pattern and decreased analytic processing, in the condition of increased task engagement (i.e. in the presence of an observer) and in the condition of low task engagement (i.e. in a lone condition) the manipulation of challenge had no impact on the cardiovascular responses. At the same time that the body fails to react to the situation it also fails to impact information-processing. Our fourth experiment offers clear evidence of a dissociation of familiarity effects from challenge effects. When we replicated experiment one with the same manipulation of task engagement, the pattern of results differed from the one that manipulated challenge. The manipulation of familiarity increased the cardiovascular pattern of challenge as expected 
only for those who were task-engaged (i.e. those in the presence of an observer). However, the familiarity impact on information-processing occurred regardless of the level of task engagement (i.e. if participants were in the presence of an observer or alone).

The evidence provided from these experiments clearly suggests that, as expected, familiarity is related to the motivational state of challenge. In addition, it is clear that both familiarity and challenge reduce analytic processing and increase the cardiovascular markers associated with that motivational state. However, our data also suggest that they may not be part of the same process at least directly. The familiarity impact on how we process information is independent of degree of task engagement. However task engagement is a necessary condition for familiarity to be associated with a challenge motivational state, which in this case is also related to less engagement in deep processing.

Although these results corroborate our general hypothesis that challenge and familiarity are closely related, they suggest that the association of familiarity with motivational challenge may not occur via the experience of personal resources availability as we previously considered. Our original idea was that both cues would signal the availability of resources to deal with the task influencing in the same way subsequent processing. Since data suggest that this may not be the case, one possibility may be that even considering that resources availability issues are relevant to both experiences, they do not map the same information. In fact, as we referred to in the beginning of this dissertation, familiarity is expected to provide individuals with resources to deal with a task, whereas the motivational state of challenge is activated by evaluation of those resources. Whereas familiarity may signal task-mastery directly, challenge encompasses an evaluation process that can occur consciously or without individuals' awareness but regardless needs task engagement. Future studies should address this hypothesis by manipulating resources availability directly and the engagement on the evaluation process in order to clarify the relevance of these factors.

In addition to the dissociation promoted by task engagement on the effects promoted by manipulation of familiarity and challenge on information processing, we found, no mediation of the effects by the physiological measures of challenge in the last two experiments. This might have occurred because familiarity and challenge simply do not relate with one another. Thus, the absence of mediation in experiment one seems to suggest that the effects of familiarity are not dependent on the activation of challenge. However, it is odd that no mediation is observed in experiment two. Challenge manipulation has an impact on information processing, and promoted congruent physiological reactions. However, the 
latter does not mediate the first effect. This suggests that what we are manipulating and what we are measuring are not the same thing. Although the two are related they do not overlap much in terms of variance, and they differ on the effect on information processing. Assuming that challenge incorporates an evaluation of two components - internal resources and situational demands, we hypothesize that it is just one of the components that is driving the effect. Thus, one possible reason for the absence of mediation in the first two experiments may be due to the different sensitivity of the effect and measure to the evaluation components.

Other possible reasons why no mediation is found in experiment 2 are methodological. Attitude-changes and challenge measures are never accessed simultaneously, but rather sequentially (separate phases) and in different phases of the process. So even if both are impacted by the manipulations, other variables can be influencing challenge motivation in such a way that it looses its link to what impacted attitude-changes. Another methodological reason may be the fact that the measure we use to test mediation is not the most appropriate one. On this note, recall that our unitary index used to test the mediation is a composite measure of $\mathrm{CO}$ and TPR. But since, HR and VC are also involved in the SAM and HPA axis activation, responsible for challenge and threat activation, maybe this index is somehow incomplete by leaving these two important measures out of the index.

It is also possible that the mediation could be in a different direction than the one we predicted. Recall that we hypothesized that the markers of the motivational state of challenge could explain to some degree the engagement in non-analytic processing. However, it is also possible that the engagement in non-analytic processing is somehow explained by the markers of the motivational state. Even though this is an interesting and relevant question, our data cannot address it because of the paradigm we used. We used a between-subject paradigm to observe the impact of our independent measures on information-processing, looking specifically to how individuals reacted to different strong and weak persuasive messages. To investigate the possibility of this mediation, future studies should use of different paradigm that allows the measurement of information processing within-subject.

But even if these are questions to clarify in future studies, it is still true that our experiments suggest clearly that familiarity and challenge effects are dissociated by task engagement - so the effect of familiarity in low involvement (low task engagement) cannot be in any way explained by the activation of a motivational state of challenge. Being both 
familiarity and challenge associated with a psychological state that signals superficial processing to be an adequate way to deal with a task, one only occurs when the task is engaging.

Why might this be so? As we suggested previously, the explanatory variable may be the need for an evaluation of resources and demands. Even though familiarity and challenge have the same impact on the modes of processing and both experiences have characteristics in common, familiarity impact is not assumed to be explained by a mechanism that encompasses engagement on such an evaluation process. The effect of familiarity on processing occurs because encoding of information matches retrieval promoting fluency of processing, experienced as a feeling of previous encountering of familiarity. This feeling signals no need to engage in deep processing. There is no need to attend to the specificities of situation (other than being merely exposed to a stimulus). So familiarity is activated by simple perception, not encompassing any type of evaluative process, specially the one that is associated with comparison between one's resources and demands. That would necessitate a different type of relationship with the situation - one that motivates the individual to engage in those evaluations. So if we increase task engagement, we should expect this comparison to be potentiated and challenge to be activated. And even though the BPS model posits that this process may occur unconsciously, being affected for instance by affect (WeisbuchRemington et al., 2005), it is always assumed that an evaluation occurs and that it encompasses resources and demands. Evaluation is necessary and moderates challenge and threat activation. Note, however, that when challenge is promoted, the accessibility to resources is also promoted and this may promote the feeling of familiarity (as presupposed by the FARM model) that is known to be associated with less analytic processing. If this is so, instead of expecting familiarity effects to be mediated by challenge indexes, we should expect challenge effects on information processing to be mediated by feeling of familiarity. Thus, a corollary of this hypothesis is that the internal experience defined as a feeling of familiarity - being automatically activated by the detection of the situation as familiar -may be incorporated in the state of challenge. Familiarity may be integrated in the experience of challenge in the sense that they both share a similar internal state defined by the detection of greater resources available, being challenge activated by a subsequent evaluation of the demands as fewer.

This hypothesis suggests that the relationship between familiarity and challenge is not a perfect match - familiarity only requires the internal experience to signal superficial 
processing and challenge adds to it the consideration of the external situation. In order to directly address this hypothesis, future studies should orthogonally manipulate the level of demands of a task and level of familiarity. Whereas the impact of familiarity is translated by a main effect of this variable, challenge effects should be associated with a specific interaction. By doing that we contrast the hypothesis that the internal detection of resources always leads to decreased processing (independently of evaluation of the external situation) with the hypothesis that this internal detection of resources only leads to decreased processing when the external task-demands are low. Although not expected, by the hypothesis here defined, it would be interesting to determine if a disruption of familiarity effects is caused by increase of task-demands. This would mean that individuals neglect the internal signal and still process information analytically because their evaluation of higher task-demands (more likely to result in a threat condition) disrupts the familiarity signal. Recall that in our experiments, more analytic processing was observed in the threat condition. To fully test the hypothesis we have been raising, it would be insightful to add a manipulation of a variable assumed to reduce the level of evaluation (e.g. level of distraction; low self monitoring etc.). In these conditions threat is not assumed to be able to disrupt familiarity effects.

Alternative explanations may however be raised. The relationship between familiarity and challenge and their impact on processing may be explained by a third variable, such as mood. The literature supports this possibility by demonstrating that the three types of relationships, able to explain the mediation (see Baron \& Kenny, 1986), are present. As demonstrated by Garcia-Marques (1999, 2009), familiarity is defined as feeling with positive valence. Similarly, challenge is associated with a positive affective state (Blascovich \& Mendes, 2000). A positive mood state has been shown to decreases by itself analytic processing (Bohner, Crow, Erb \& Schwarz, 1992; Mackie \& Worth, 1989). Thus, it is possible that mood mediates the effects of familiarity and challenge on processing by being the link between the two. Unfortunately this is not corroborated by our data which revealed an absence of mediation of participants' ratings of mood on the effects of both challenge and familiarity in information processing. Of course this might be due to a methodological problem, since participants' ratings of mood were assessed at the very end of the experiment after participants were detached from all the physiological equipment. Because there is some level of unpleasantness (e.g. itchiness) associated with removing this equipment from participants' body, it is possible that participants' ratings were biased by our 
procedure. This may be a reason worthwhile to persecute the in future studies. But an additional feature of our data also puts the explanation into question. How would mood explain the dissociative effects of task engagement in both variables? In addition, mood effects are assumed to disappear whenever task engagement is increased (e.g. Smith \& Shaffer, 1991).

\section{Contributions for the models.}

\section{BPS model.}

The research we present in this dissertation offers a cognitive insight on the BPS model by being the very first attempt to relate challenge and threat motivational states with dual process models of information-processing. Contrary to the usual but not universal finding that challenge leads to better performances, our data suggests that this is not always the case - challenge participants performed poorly on an information processing task. Of course, within an information-processing perspective, it is necessary to reframe the notion of "better" and "worse" performance because analytic processing is not better or worse than superficial processing, their difference being qualitative rather than quantitative. When we take such a perspective to interpret the common findings in the BPS literature, some results are actually consistent with ours. For example, evidence showing that challenge increases speed for judgments of preference tasks (Blascovich et al, 1993) may indicate that nonanalytic processing, typically associated with shorter reaction times, is being used. In line with this, Hunter's (2001) investigation clearly suggest that when more controlled processes are required, challenge results in worse performances as demonstrated by a small amount of correctly recalled words from a previously seen list. However, in some circumstances, where for example individuals have to deliver a speech after a downward social comparison cooperative task (Mendes et al., 2002), challenge generally improves performance. These discrepancies suggest that the type of task possibly moderates the effects, a hypothesis worthwhile to explore in future studies.

Additional contributions regarding the BPS model involve the evidence that even though challenge is associated with increased task resources relative to task demands and cognitive resources are needed to process information more systematically, the relatively greater amount of resources during challenge motivation is not translated into deeper processing. Congruent with our findings, some literature in cognitive psychology has shown that having more resources does not necessarily lead to analytic processing. For example, 
Lavie and colleagues (1995; Lavie, Hirst, de Fockert \& Viding, 2004) have shown that some level of cognitive load is necessary for selective attention to increase cognitive performance. Others like Baron (1986) have suggested that some level of distraction is necessary to focus individuals on what is the relevant information to perform a task correctly. Our data is the preliminary evidence that questions if the definition of "personal resources" conveyed in the BPS model maps or not the "cognitive resources" needed to process information.

\section{FARM model.}

The research conducted here not only offers further support for the FARM model but also offers some insights about the role of motivational processes. As it has been demonstrated in the literature, motivation is an important variable because it moderates FARM effects. Independently of the levels of familiarity with the task, less motivated performers are less likely to engage in analytic processing, whereas, highly motivated individuals are much more likely to (e.g. Claypool et al., 2004). Familiarity effects are made clear whenever motivation levels do not disrupt or potentiate analytic processing. However, our findings, suggest that not all kinds of motivation (e.g. involvement with the issue) moderate the effect. At least the type of task engagement brought by the presence of an observer, does not impact the FARM effects - information was always processed similarly whether participants were or not in the presence of others.

Additional contribution to the FARM is the evidence demonstrating that the effects of familiarity on information-processing have physiological correlates associated with it. Other researchers have already demonstrated this by showing that the presentation of famous familiar faces evoke larger skin conductance responses (Tranel, Fowles \& Damasio, 1985); mere exposure to familiar faces compared to unfamiliar faces increases electrodermal reactions (Channouf \& Rouibah, 1997); or that processing of repeated stimuli is associated with decreases in neural activity (Ishai, Bikle \& Ungerleider, 2006). However, as far as we know, no past evidence for physiological correlates was associated with familiarity with a persuasive message. The correlates we found in our experiments suggest that familiarity of a message is associated with the sympathetic neural and adrenal medullary (SAM) axis activation (marked by increases in cardiac output and decreases or no changes in total peripheral resistance), being this manifestations circumscribed to motivated task performance situations. In order to provide more evidence on this relationship, it would be 
interesting to approach physiological correlates (and what are they) outside motivated tasksituations.

\section{Additional contributions to the Social Facilitation literature.}

The idea that the presence of others increases task engagement is not new in the social facilitation literature. Authors have thoroughly demonstrated that the presence of others is motivating and increases the levels of effort allocated in the task (Bond, 1982; Blascovich et al., 1999; Zajonc, 1965, 1980, for reviews see, Aiello \& Douhit, 2001; Guerin, 1993). However, our findings demonstrate that this increased effort is not necessarily translated into motivation to process deeply information. This could mean that the type of task engagement promoted by the presence of others is different than the type of engagement necessary to elaborate information deeply. In fact, it is possible that the type of task engagement outlined in the social facilitation literature is merely motivational and not cognitive, as its dissociative effect in our experiments seems to suggest.

A hypothesis worth pursuing in future studies is one associated with our interpretation of the dissociation effect. As we suggested above, one possible explanation for the dissociative effect may be a greater tendency to attend to the context when others are present. Congruent with this hypothesis, in challenge situations the individuals in the alone condition may be prevented from evaluating the demands of the situation. Thus, the presence of others may moderate the activation of a challenge state because individuals are prone to attend to those social features of the situation. In the presence of others, individuals, assumed to attribute importance to the task, may be only more prone to attend the demands of the situation in order to evaluate it as challenge or threat. As such, it is possible that the presence of others, instead of engaging individuals in a task, makes them more sensitive to the context. In line with this hypothesis, Fonseca and Garcia-Marques (2010) have demonstrated that, compared to an alone situation, the presence of others increases the amount of contextrelated free-associations (experiment 1) and the accuracy for estimation judgments that require the integration of contextual information (experiment 3). This context-sensitivity hypothesis can also be found in social facilitation evidence that shows that accompanied individuals have better recall for contextual aspects of the task-situation, (Sanders, Baron \& Moore, 1978) and make greater use of contextual cues when forming an impression of a target (Thomas et al., 2002). Even though this is an interesting hypothesis, future research should clarify why in situations where this evaluative process associated with motivational 
state is not enhanced, the impact of variable such as familiarity on processing remains the same.

The set of 4 studies we present in this thesis is the very first attempt to empirically test the relationships between two different and distant models. The results we obtained here, even though they do not offer a fully and final response to our goals, suggest future avenues, worthwhile to pursue, for exploring the interconnection between motivational and cognitive impact of social human behavior. 


\section{References}

Aiello, J. R. \& Douhit, E. A. (2001). Social facilitation from Triplett to electronic performance monitoring. Group Dynamics: Theory, Research and Practice, 5, $162-$ 180.

Alter, A. L., \& Oppenheimer, D. M. (2006). Predicting short-term stock fluctuations by using processing fluency. Proceedings of the National Academy of Sciences, 103, 9369-9372.

Alter, A. L., \& Oppenheimer, D. M. (2009). Uniting the Tribes of Fluency to Form a Metacognitive Nation. Personality and Social Psychology Review, 13, 219-235.

Alter, A. L., Aronson, J., Darley, J. M., Rodriguez, C., \& Ruble, D. N. (2010). Rising to the threat: Reducing stereotype threat by reframing the threat as a challenge. Journal of Experimental Social Psychology, 46, 166-171.

Alter, A. L., Oppenheimer, D. M., Epley, N., \& Eyre, R. N. (2007). Overcoming intuition: Metacognitive difficulty activates analytic reasoning. Journal of Experimental Psychology: General, 136, 569-576.

Aronson, J., \& McGlone, M. (2009). Stereotype and social identity threat. In T. Nelson (Ed.), The handbook of prejudice, stereotyping, and discrimination (pp. 153-178). New York: Guilford.

Baron, R. (1986). Distraction-conflict theory: progress and problems. In L. Berkowitz (Ed.), Advances in Experimental Social Psychology (pp. 1- 40), vol. 19. New York: Academic Press.

Baron, R. M., \& Kenny, D. A. (1986). The moderator-mediator variable distinction in social psychological research: Conceptual, strategic, and statistical considerations. Journal of Personality and Social Psychology, 51, 1173-1182.

Begg, I., \& Armour, V. (1991). Repetition and the ring of truth: Biasing comments. Canadian Journal of Behavior Science, 23, 195-213.

Blascovich, J. (2008). Challenge and threat. In A. J. Elliot (Ed.), Handbook of approach and avoidance motivation (pp. 431 - 445). New York: Psychology Press.

Blascovich, Ernst, J. M., Tomaka, J., Kelsey, R.M., Salomon, K. L. \& Fazio, R. H. (1993). Attitude Accessibility as a Moderator of Autonomic Reactivity During Decision Making. Journal of Personality and Social Psychology, 64, 165-176.

Blascovich, J. \& Katkin, E. S. (Eds). (1993). Cardiovascular reactivity to psychological stress and disease. Washington, DC: American Psychological Association.

Blascovich, J., \& Mendes, W. B. (2000). Challenge and threat appraisals: The role of affective cues. In J. Forgas (Ed.), Feeling and thinking: The role of affect in social cognition (pp. 59-82). Cambridge, UK: Cambridge University Press.

Blascovich, J. Mendes, W. B., Hunter, S. \& Lickel, B., \& Kowai-Bell, N. (2001). Perceiver threat in social interactions with stigmatized others. Journal of Personality and Social Psychology, 80, 253-267.

Blascovich, J., Mendes, W., Hunter, S. \& Salomon, K. (1999). Social facilitation as challenge and threat. Journal of Personality and Social Psychology, 77, 68-77.

Blascovich, J., Mendes, W.B., \& Seery, M. (2002). Intergroup encounters and threat: A multi-method approach. In D. Mackie \& E. Smith (Eds.). From prejudice to intergroup emotions: Differentiated reactions to social groups (pp. 89-110). New York: Psychology Press. 
Blascovich, J., Mendes, W. B., Tomaka, J., Salomon, K., \& Seery, M.D. (2003). The robust nature of the biopsychosocial model of challenge and threat: A reply to Wright and Kirby. Personality and Social Psychology Review, 7, 234-243.

Blascovich, J., \& Seery, M. D. (2006). Visceral and somatic indexes of social psychological constructs. In A. Kruglanski \& E. T. Higgins (Eds.), Social psychology: Handbook of basic principles (2nd ed., pp. 19 - 38). New York: Guilford Press.

Blascovich, J., Seery, M. D., Mugridge, C. A., Norris, R. K., \& Weisbuch, M. (2004). Predicting athletic performance from cardiovascular indexes of challenge and threat. Journal of Experimental Social Psychology, 40, 683-688.

Blascovich, J., \& Tomaka, J. (1996). The biopsychosocial model of arousal regulation. In M. P. Zanna (Ed.), Advances in experimental social psychology (Vol. 28, pp. 1-51). San Diego, US: Academic Press.

Bohner, G., Crow, K., Erb, H.-P., \& Schwarz, N. (1992). Affect and persuasion: Mood effects on the processing of message content and context cues and on subsequent behavior. European Journal of Social Psychology, 22, 511-530.

Bond, C. F. (1982). Social Facilitation: a self-presentational view. Journal of Personality and Social Psychology, 42, 1042-1050.

Brehm, J. W., \& Self, E. (1989). The intensity of motivation. In M. R. Rozenweig \& L. W. Porter (Eds.), Annual review of psychology (pp. 109-131). Palo Alto, CA: Annual Reviews.

Cacioppo, J. T., \& Petty, R. E. (1979). Effects of message repetition and position on cognitive response, recall, and persuasion. Journal of Personality and Social Psychology, 37, 97-109.

Cacioppo, J. T., \& Petty, R. E. (1989). Effects of message repetition on argument processing, recall and persuasion. Basic and Applied Social Psychology, 10, 3-12.

Chaiken, S. (1987). The heuristic model of persuasion. In M. P. Zanna, J. M. Olson, \& C. P. Herman (Eds.), Social influence: The Ontario Symposium (Vol. 5, pp. 3-39). Hillsdale, NJ: Erlbaum.

Chaiken, S., \& Trope, Y. (Eds.) (1999). Dual-process theories in social psychology. New York, NY: Guilford Press.

Channouf, A. \& Rouibah, A. (1997). Suboptimal familiar faces exposure and electrodermal reactions. Anuario de Psicologia, 74, 85-97.

Chen, S., \& Chaiken, S. (1999). The heuristic-systematic model in its broader context. In S. Chaiken \& Y. Trope (Eds.), Dual-process theories in social psychology (pp. 73-96). New York: Guilford.

Chen, S., Shechter, D., \& Chaiken, S. (1996). Getting at the truth or getting along: Accuracyversus impression-motivated heuristic and systematic processing. Journal of Personality and Social Psychology, 71, 262-275.

Claypool, H. M., Mackie, D. M., Garcia-Marques, T., McIntosh, A., \& Udall, A. (2004). The effects of personal relevance and repetition on persuasive processing. Social Cognition, 22, 310-335.

Cottrel, N. B., Wack, D. L., Sekerak, G. J. \& Ritlle, R. H. (1968). Social facilitation of dominant responses by the presence of an audience and the mere presence of others. Journal of Personality and Social Psychology, 9, 245-250.

Cox, T. (1978). Stress. Baltimore: University Park Press.

Dewhurst, S.A., \& Hitch, G.J. (1997). Illusions of familiarity caused by cohort activation. Psychonomic Bulletin and Review, 25, 484-491.

Dienstbier, R. A. (1989). Arousal and physiological toughness: Implications for mental and physical health. Psychological Review, 96, 84-100. 
Drach-Zahavy, A., \& Erez, M. (2002). Challenge versus threat effects on the goalperformance relationship. Organizational Behavior and Human Performance, 88, 667-682.

Eagly, A. H., \& Chaiken, S. (1993). The psychology of attitudes. Fort Worth, TX: Harcourt Brace Jovanovich.

Eliezer, D., Major, B., \& Mendes, W. B. (2010). The costs of caring: Gender identification increases threat following exposure to sexism. Journal of Experimental Social Psychology, 46, 159-165.

Evans, J. (2008). Dual processing accounts of reasoning, judgment and social cognition. Annual Review of Psychology, 59, 255-78.

Fazio, R. H., Blascovich, J., \& Driscoll, D. M. (1992). On the functional value of attitudes: The influence of accessible attitudes upon the ease and quality of decision-making. Personality and Social Psychology Bulletin, 18, 388-401.

Feinberg, J. \& Aiello, J. (2009) The Effect of Challenge and Threat Appraisals Under Evaluative Presence. Journal of Applied Social Psychology, 40, 2071 - 2104.

Fonseca, R. \& Garcia-Marques, T. (2010). Back to basics: social facilitating situated cognition (under review).

Garcia-Marques, T. (1999). The mind needs the heart: The mood-as-regulation-mechanism hypothesis as an explanation for the impact of mood on processing. Unpublished doctoral dissertation, University of Lisbon, Portugal.

Garcia-Marques, T. (2003). A regulação da activação de diferentes modos de processamento de informação: O papel do "sentimento de familiaridade". Análise Psicológica, 3, 267-285.

Garcia-Marques, T. (2009). Se sinto como familiar sinto como positivo! Interferência da familiaridade no processo avaliativo. Análise Psicológica, 4, 447-454.

Garcia-Marques, T., \& Mackie, D. M. (2000). The positive feeling of familiarity: Mood as an information processing regulation mechanism. In H. Bless \& J. P. Forgas (Eds.), The message within: The role of subjective experience in social cognition and behavior. (pp. 240-261). New York, NY US: Psychology Press.

Garcia-Marques, T., \& Mackie, D. M. (2001). The feeling of familiarity as a regulator of persuasive processing. Social Cognition, 19(1), 9-34.

Garcia-Marques, T., \& Mackie, D. M. (2007). Familiarity impacts person perception. European Journal of Social Psychology, 37, 839-855.

Garcia-Marques, T., Mackie, D. M., Claypool, H. M., \& Garcia-Marques, L. (2010). Is it familiar or positive? Mutual Facilitation of response latencies. Social Cognition, 28, 205-218.

Greenwald, A. G. (1968). Cognitive learning, cognitive response to persuasion, and attitude change. In A. G. Greenwald, T. C. Brock, and T. M. Ostrom (Eds.), Psychological foundations of attitudes (pp. 147-170). New York: Academic Press.

Guerin, B. (1993). Social facilitation. Cambridge, England: Cambridge University Press.

Halberstadt, J. \& Catty, S. (2008). Analytic Thought Disrupts Familiarity-Based Decision Making. Social Cognition, 26, 755-765.

Hunter, S. B. (2001). Performance under pressure: The impact of challenge and threat states on information processing. Unpublished doctoral dissertation, University of California, Santa Barbara.

Higgins, E. T. (1989). Knowledge accessibility and activation: subjectivity and suffering from unconscious sources. In J. S. Uleman, \& J. A. Bargh (Eds.), Unintended thought (pp. 75-123). New York, NY: Guilford Press. 
Ishai, A., Bikle, P.C., Ungerleider L.G. (2006). Temporal dynamics of face repetition suppression. Brain Research Bulletin 70, 289-295.

Jacoby, L. L. (1991). A process dissociation framework: Separating automatic from intentional uses of memory. Journal of Memory and Language, 30, 513-541.

Jacoby, L. L., \& Kelley, C. M. (1990). An episodic view of motivation: Unconscious influences of memory. In E. T. Higgins \& R. M. Sorrentino (Eds.), Handbook of motivation and cognition (Vol. 2, pp. 451-480). New York: Guilford.

Jacoby, L. L., Kelley, C, Brown, J., \& Jasechko, J. (1989). Becoming famous overnight: Limits on the ability to avoid unconscious influences of the past. Journal of Personality and Social Psychology, 56, 326-338.

Jacoby, L.L., Woloshyn, V., \& Kelley, C. (1989). Becoming famous without being recognized: Unconscious influences of memory produced by dividing attention. Journal of Experimental Psychology: General, 118, 115-125.

Johnston, W. A., \& Hawley, K. J. (1994). Perceptual inhibition of expected inputs: The key that opens closed minds. Psychonomic Bulletin and Review, 1, 56-72.

Kelsey, R.M., Blascovich, J., Leiten, C. L., Schneider, T. R., Tomaka J. \& Wiens, S. ( 2000). Cardiovascular reactivity and adaptation to recurrent psychological stress: The moderating effects of evaluative observation. Psychophysiology, 37, 748-756.

Labroo, A. A., Dhar, R., \& Schwarz, N. (2008). Of frowning watches and frog wines: Semantic priming, perceptual fluency, and brand evaluation. Journal of Consumer Research, 34, 819-831.

Lazarus, R. S, (1966). Psychological Stress and the Coping Process. New York: McGrawHill.

Lazarus, R.S. (1991). Progress on a cognitive-motivational-relational theory of emotion. The American psychologist, 46, 819-834.

Lazarus, R.S., \& Folkman, S. (1984). Stress, appraisal, and coping. New York: Springer.

Lavie, N. (1995). Perceptual load as a necessary condition for selective attention. Journal Experimental Psychology: Human Perception Performance, 21,451-468.

Lavie, N., Hirst, A., Fockert, Jan W. \& Viding, E. (2004). Load Theory of Selective Attention and Cognitive Control. Journal of Experimental Psychology General, 133, 339-354.

Light, K. C. (1981). Cardiovascular responses to effortful active coping: Implications for the role of stress in hypertension development.Psychophysiology, 17, 243-252.

Mackie, D. M., \& Worth, L. T. (1989). Processing deficits and the mediation of positive affect in persuasion. Journal of Personality and Social Psychology, 57, 27-40.

Maier, K. J., Waldstein, S. R., \& Synowski, B. A. (2003). Relation of cognitive appraisal to cardiovascular reactivity, affect, and task engagement. Annals of Behavioral Medicine, 26, 32-41.

McGlone, M. S., \& Tofighbakhsh, J. (2000). Birds of a feather flock conjointly (?): Rhyme as reason in aphorisms. Psychological Science, 11, 424-428.

Mendes, W.B., Blascovich, J., Hunter, S.B., Lickel, B., \& Jost, J.T. (2007). Threatened by the Unexpected: Physiological Responses during Social Interactions with Expectancy-Violating Partners. Journal of Personality and Social Psychology. 92, $698-716$.

Mendes, W.B., Blascovich, J., Lickel, B., \& Hunter, S. (2002). Challenge and threat during interactions with White and Black men. Personality and Social Psychology Bulletin, 28, 939-952. 
Mendes, W.B., Blascovich, J., Major, B., \& Seery, M. (2001). Effects of social comparisons on challenge and threat reactivity. European Journal of Social Psychology, 31, 477479.

Mendes, W.B., Major, B., McCoy, S., \& Blascovich, J. (2008). How attributional ambiguity shapes physiological and emotional responses to social rejection and acceptance. Journal of Personality and Social Psychology, 94, 278-291.

Mendes, W. B., Weisbuch, M., Seery, M. D., \& Blascovich, J. (October, 2002). Challenge and threat following subliminal presentations of valenced religious pictures. Poster session presented at the annual meeting of the Society for Psychophysiological Research, Washington, DC.

Moons, W., Mackie, D. \& Garcia-Marques, T. (2009). The Impact of Repetition-Induced Familiarity on Agreement With Weak and Strong Arguments. Journal of Personality and Social Psychology, 96, 32-44.

O'Connor, K. M., Arnold, J. A., \& Maurizio, A. M. (2010). The prospect of negotiating: Stress, cognitive appraisal, and performance. Journal of Experimental Social Psychology, 46, 729-735.

Obrist, P. A. (1981). Cardiovascular psychophysiology: A perspective. New York: Plenum Press.

Obrist, P. A., Gaebelein, C. J., Teller, E. S., Langer, A.W., Grignolo, A., Light, K. C \& McCubbin, J.A. (1978). The relationship among heart rate, carotid dp/dt, and blood pressure in humans as a function of type of stress. Psychophysiology, 15, 102-115.

Petty, R. E. \& Cacioppo, J. T. (1986). Communication and persuasion: central and peripherical routes to attitude change. New York: Spring - Verlag.

Petty, R. E., Cacioppo, J. T., \& Goldman, R. (1981) Personal involvement as a determinant of argument-based persuasion. Journal of Personality and Social Psychology, 41, 847-55.

Petty, R. E. \& Wegener, D. T. (1998). Attitude change: Multiple roles for persuasion variables. In D. Gilbert, S. Fiske, \& G. Lindzey (Eds.), The handbook of social psychology (4 ${ }^{\text {th }}$ ed., pp. 323-390). New York: McGraw-Hill.

Reder, L. M. (1987). Strategy selection in question answering. Cognitive Psychology, 19, 111-138.

Reder, L. M., \& Ritter, F. E. (1992). What determines initial feelings of knowing? Familiarity with question terms not with the answer. Journal of Experimental Social Psychology: Learning, Memory and Cognition, 18, 435-451.

Sanders, G.S., Baron, R. S. \& Moore, D. L. (1978) Distraction and social comparison as mediators of social facilitation effects. Journal of Experimental Social Psychology, 14, 291-303.

Schmader, T., Johns, M., \& Forbes, C. (2008). An integrated process model of stereotype threat effects on performance. Psychological Review, 115, 336-356.

Schunn, C. D., Reder, L. M., Nhouyvanisvong, A.,Richards, D. R., \& Stroffolino (1997). To calculate or not to calculate: A source activation confusion model of problem familiarity's role in strategy selection. Journal of Experimental Psychology: Learning, Memory, and Cognition, 23, 3-29.

Schwarz, N. (2004). Meta-cognitive experience in consumer judgment and decision making. Journal of Consumer Psychology, 14, 332-348.

Schwarz, N., \& Clore, G. L. (1996). Feelings and Phenomenal Experiences. In E. T. Higgins, \& A. W. Kruglanski (Eds.), Social psychology. Handbook of basic principles (pp. 433-465). New York, NY: Guilford Press. 
Seery, M., Blascovich, J.. Weisbuch, M., Vick, S.B. (2004). The relationship between selfesteem, self-esteem stability, and cardiovascular reactions to performance feedback. Journal of Personality and Social Psychology, 87, 133-145.

Seery, M. D., Weisbuch, M., \& Blascovich, J. (2009). Something to gain, something to lose: The cardiovascular consequences of outcome framing. International Journal of Psychophysiology, 73, 308-312.

Shah, A. K., \& Oppenheimer, D. M. (2007). Easy does it: The role of fluency in cue weighting. Judgment and Decision Making, 2, 371-379.

Sherwood, A., Allen, M. T., Fahrenberg, J., Kelsey, R. M., Lovallo, W. R., \& van Dooren, L. J. P. (1990). Methodological guidelines for impedance cardiography. Psychophysiology, 27, 1-23.

Smith, E. R. (1994). Procedural knowledge and processing strategies in social cognition. In R. S. Wyer, \& T. K. Srull (Eds.), Handbook of social cognition (2nd ed., Vol. 1, pp. 99-151). Hillsdale, NJ: Lawrence Erlbaum Associates Inc.

Smith, E. R., \& DeCoster, J. (2000). Dual-process models in social and cognitive psychology: Conceptual integration and links to underlying memory systems. Personality and Social Psychology Review, 4, 108-131.

Smith, E. R., Miller, D. A., Maitner, A. T., Crump, S. A., Garcia-Marques, T., \& Mackie, D. M. (2006). Familiarity can increase stereotyping. Journal of Experimental Social Psychology, 42, 471-478.

Smith, S. M. \& Schaffer, D. R. (1991).The effects of good moods on systematic processing: "Willing but not able, or able but not willing? Motivation and Emotion, 15, 243 279.

Song, H. \& Schwarz, N. (2008). Fluency and the detection of misleading questions: low processing fluency attenuates the Moses illusion. Social Cognition, 26, 791-799.

Taylor, S. E., Seeman, T. E., Eisenberger, N. I., Kozanian, T. A., Moore, A. N., \& Moons, W. G. (2010). Effects of a supportive or unsupportive audience on biological and psychological responses to stress. Journal of Personality and Social Psychology, 98, 47-56.

Thomas, S., Skitka, L. J., Christen, S., \& Jurgena, M. (2002). Social facilitation and impression formation. Basic and Applied Social Psychology, 24, 67-70.

Tomaka, J., \& Blascovich, J. (1994). Effects of justice beliefs on cognitive appraisal of and subjective, physiological, and behavioral responses to potential stress. Journal of Personality Social Psychology, 67, 732-740.

Tomaka, J., Blascovich, J., Kelsey, R. M., \& Leitten, C. L. (1993). Subjective, physiological, and behavioral effects of threat and challenge appraisal. Journal of Personality and Social Psychology, 65, 248-260.

Tomaka, J., Blascovich, J., Kibler, J., \& Ernst, J. M. (1997). Cognitive and Physiological Antecedents of Threat and Challenge Appraisal. Journal of Personality and Social Psychology, 73, 63-72.

Tomaka, J., \& Palacios-Esquivel, R. L. (1997). Motivational systems and stress-related cardiovascular reactivity. Motivation and Emotion, 21, 275-296.

Tomaka, J. Palacios, R., Schneider, K., Colotla, M., Concha, J. \& Herrald, M. (1999). Assertiveness predicts threat and challenge reactions to potential stress among women. Journal of Personality and Social Psychology, 76, 1008-1021.

Tormala, Z. L., Briñol, P. \& Petty, R. E. (2006). When credibility attacks: The reverse impact of source credibility on persuasión. Journal of Experimental Social Psychology, 42, 684-691. 
Tranel, D., Fowles, D.C., \& Damasio, A.R. (1985). Electrodermal discrimination of familiar and unfamiliar faces: A methodology. Psychophysiology, 22(4), 403-408.

Vick, S.B., Seery, M.D., Blascovich, J. \& Weisbuch, M. (2008) The effect of gender stereotype activation on challenge and threat motivational states. Journal of Experimental Social Psychology, 44, 624-630.

Weisbuch-Remington, M., Mendes, W.B., Seery, M.D., \& Blascovich, J. (2005). The nonconscious influence of religious symbols in motivated performance situations. Personality and Social Psychology Bulletin, 31, 1203-1216.

Whittlesea, B. W. A. (1993). Illusions of familiarity. Journal of Experimental Psychology: Learning, Memory, \& Cognition, 19, 1235-1253.

Whittlesea, B. W. A., \& Leboe, J. P. (2003). Two fluency heuristics (and how to tell them apart). Journal of Memory and Language, 49, 62-79.

Whittlesea, B. W. A., \& Williams, L. D. (2000). The source of feelings of familiarity: The discrepancy-attribution hypothesis. Journal of Experimental Psychology:Learning, Memory, and Cognition, 26, 547-565.

Whittlesea, B. W., Jacoby, L. L., \& Girard, K. (1990). Illusions of immediate memory: Evidence of an attributional basis for feelings of familiarity and perceptual quality. Journal of Memory \& Language, 29, 716-732.

Winkielman, P. \& Cacioppo, J. (2001). Mind at ease puts a smile on the face: Psychophysiological evidence that processing facilitation leads to positive affect. Journal of Personality and Social Psychology, 81, 989-1000.

Winkielman, P., Halberstadt, J., Fazendeiro, T., \& Catty, S. (2006). Prototypes are attractive because they are easy on the mind. Psychological Science, 17, 799-808.

Wood, W., Kallgren, C. A., \& Preisler, R. M. (1985). Access to attitude-relevant information as a determinant of persuasion: The role of message attributes. Journal of Experimental Social Psychology, 21, 73-85.

Worth, L. T., \& Mackie, D. M. (1987). Cognitive mediation of positive mood in persuasion. Social Cognition, 5, 76-94.

Wright, R. A., \& Kirby, L. D. (2001). Effort determination of cardiovascular response: An integrative analysis with applications in social psychology. In M. P. Zanna (Ed.), Advances in experimental social psychology (Vol. 33, pp. 255-307). San Diego, CA: Academic.

Wright, R. A., \& Kirby, L. D. (2003). Cardiovascular Correlates of Challenge and Threat Appraisals: A Critical Examination of the Biopsychosocial Analysis. Personality and Social Psychology Review, 31, 216 - 233.

Zajonc, R. B. (1965). Social Facilitation. Science, 149, 269-274.

Zajonc, R. B. (1968). Attitudinal effects of mere exposure. Journal of Personality and Social Psychology Monographs, 9(2, Pt. 2).

Zajonc, R. B. (1980). Compresence, In P. B. Paulus (Ed.), Psychology of group influence (pp. 35-60). Hilldsale, NJ: Erlbaum. 


\section{Appendix A: Persuasive Messages}

\section{Weight Loss Centers: Strong Version}

"I am strongly in favor of Weight Loss Centers as places where people can safely and effectively lose weight. There are at least a couple of reasons for advocating such a position. First of all, it is important to understand that the goal of the most commercial diet programs is not, and will never be, simply to help people to lose weight. Their special concern is in teaching people how to maintain a good and healthy lifestyle. Second, most Weight Loss Centers have the advantage of having qualified personnel on hand. Weight Loss Center counselors are specially trained to deal with their often vulnerable clients. These instructors not only have to have prior relevant knowledge but must also undergo intense training sessions sponsored by the Center.

One important aspect of this training is that weight loss counselors at most centers are instructed in the use of behavior modification techniques. These are techniques that teach participants how to reward behavior that promotes their goals and to reduce behaviors that interfere with losing weight and becoming healthier. These techniques also effectively increase client's self-esteem and feeling of control. This work is usually supervised by psychologists or behavior therapists.

In addition to weight loss counselors, most diet centers employ licensed fitness trainers. These fitness trainers develop complete programs of exercises adapted to each case. These fitness programs are designed and adapted for the general overweight population, preventing any dangerous use of exercise for these people. We have to remember that not all forms of exercise are good in all phases of the process of losing weight. Commercial weight loss centers thus have fully developed programs of exercise that are safe and efficient in attaining the proper goals.

Weight loss Centers are also monitored full time by qualified medical professionals. In these ways these programs ensure people that all aspects related with weight loss are paid careful attention: nutrition, exercise, psychological support, and education related to better and healthier lifestyles. Perhaps this is the reason that over the years a great number of these centers have demonstrated that they produce the kind of lifestyle changes necessary for clients not only to safely lose weight but more importantly to keep the weight off.

For all these reasons I believe that not only people can safely and effectively lose weight with these programs, but also that this is one of the best ways of doing it."

\section{Weight Loss Centers: Weak Version}

"I am strongly in favor of weight loss centers as places where people can safely and effectively lose weight. There are at least a couple of reasons for advocating such a position. First of all, it is important to understand that if so many people use commercial weight loss programs these programs must be an effective way to lose weight. Second, we have to understand that attending the program's weekly meeting is a really good and healthy way to spend time and meet new people. A lot of overweight people might not do this if it wasn't for these centers.

One of the important aspects of these weight loss centers is that most of the clients who use commercial diet centers report little or no feeling of hunger or deprivation while participating in the program. Although there might be some complaints about some things that go on people seem to be pretty satisfied with what they are allowed to eat and drink on these programs.

I know that some commercial weight loss programs have recently been said to cause different kinds of health problems, but I believe that most of the claims of health problems arising from the general use of diet centers are unfounded and simply reflect exploitation by unscrupulous attorneys. We must remember that people who join a weight Loss center program usually expect the center to perform miracles without their having to put any time and effort into it. The people who join are also pretty vulnerable psychologically. These are just the characteristics that make the users 
of weight loss centers really easy targets for the kind of arguments used by unscrupulous attorneys who want to cause trouble for the centers while filling their own pockets.

In addition, when we compare the programs offered in weight loss centers and what people do when they try to lose weight at home alone, it turns out that the food products manufactured for specific weight loss programs are usually less expensive than comparable non-programs foods. People don't think about that when they complain about the centers. So it seems self evident the benefits of using these programs in terms of overall quality of life far outweigh the monetary cost.

For all these reasons I believe that not only people can safely and effectively lose weight with these programs, but also that this is one of the more enjoyable ways of doing it.

\section{Acid Rain: Strong Version}

"I'm strongly opposed to imposing governmental controls to minimize the effects of acid rain on the North Eastern states.

Recently completed studies have shown that most of the increase in the acidity of our lakes and atmosphere is due to increased urbanization in the northern states. Geographic changes, such as widespread deforestation, have also contributed. The deacidifying effect of large scale forest burnoffs now no longer occurs, with the result that atmospheric acidity levels have steadily climbed."

Solving these demographic and geographic problems would have a more beneficial effect than imposing controls on industry. As the installation of sulfur dioxide emissions control devices is extremely expensive such a move would be economically detrimental. American industry would be faced with a large financial burden at a time when it must focus all its financial energies on increased production to compete with ever-growing foreign competition.

A Hudson study calculates that it would cost $\$ 100$ billion to achieve a major reduction in sulfur dioxide emissions. This cost would not only be to industry, but also to the American taxpayer. As the evidence indicates that the contribution of industrial emissions to acid rain is minimal, there is no justification for engaging in a program of this expense."

\section{Acid Rain: Weak Version}

"I'm strongly opposed to imposing governmental controls to minimize the effects of acid rain on the North Eastern states.

People who think that acid rain is caused by Midwestern factories and is raining down on Eastern forests and lakes don't recognise that the material they say is in the air has probably always been there. It just happens that in the last six years they started to measure the presence of acid rain and so now it is noticeable.

Most people who live in the East have not noticed any big differences in their air or water quality which is better that in most big cities in America. If the problem were really so bad you'd think that people would be demonstrating their discontent by moving out of the area. There is no problem with the acidity in the air or water in the Midwest region, and it seems unfair to make people in the area responsible for the problems in other regions. States independence has always been an important American virtue and it should be encouraged rather than undermined. Everyone is always blaming American industry for everything, and using them as an excuse to increase bureaucracy. Factories in other countries do not have to be burdened with regulations and neither should American companies."

\section{Road Taxes Message}

"Tax rates should be increased to help repair our freeways. There are several reasons for why this action should be taken. 
First, a tax rate increase would help in the beautification of our freeways and also make them more interesting. Considering that many of our current freeways are somewhat unsightly and boring, this tax rate increase could repair some of our highways' unattractiveness. Increasing tax rates would additionally reduce the number of hitchhikers by making our highways more attractive. That is, hitchhikers would stand out against this more attractive freeway background, and as such, be easier for police to ticket these individuals. Second, the construction crews working on the repair of our freeways should increase the safety of untamed animals living in close proximity to highways. That is, the noise and commotion cause by these construction workers would deter deer and other animals from lurking near the freeways and potentially getting injured.

Finally, the government should be trusted and supported in issued related to the maintenance of our highways. The government has often done good things in the past to increase the efficiency and safety of our highways and should be trusted to do the same here." 
Appendix B: Pre-attitudinal Questionnaire.

\section{$\underline{\text { Initial Questionnaire }}$}

1. What is your gender? $\mathrm{M}$

$\mathrm{F}$

2. How old are you? year old.

3. What class are you? Freshman __ ; Sophomore _ _ Junior __ ; Senior

4. Do you have any problems related to deafness or difficult in hearing?

Yes which type?

No

To help you answer the next questions we would like you to use a feeling thermometer. Like a regular thermometer, a feeling thermometer measures all the way from cold to hot. You can use the feeling thermometer to show how "cold" or "hot" you feel about various things. If you disagree or dislike something, you can give it a "cold" rating, choosing a temperature somewhere between 0 and 49. On the other hand, if you like or agree with something you can give it a hot rating somewhere between 51 and 100 .

For instance, when you see a statement like:

\section{"The government should be stricter with people who do not recycle"}

You can select a temperature that best represents your feelings toward the issue. If you agree that the government should be stricter with people who do not recycle, you can answer with a number that represents a warm or hot feeling. The more you agree with the statement, the higher the temperature rating you can give it. If you disagree with the statement you should give it a number associated with a cold feeling. The more you disagree with the statement, the colder the rating you should give it. If you are totally indifferent to the issue you should choose a number near 50 . See the example below and start answering on the next page.

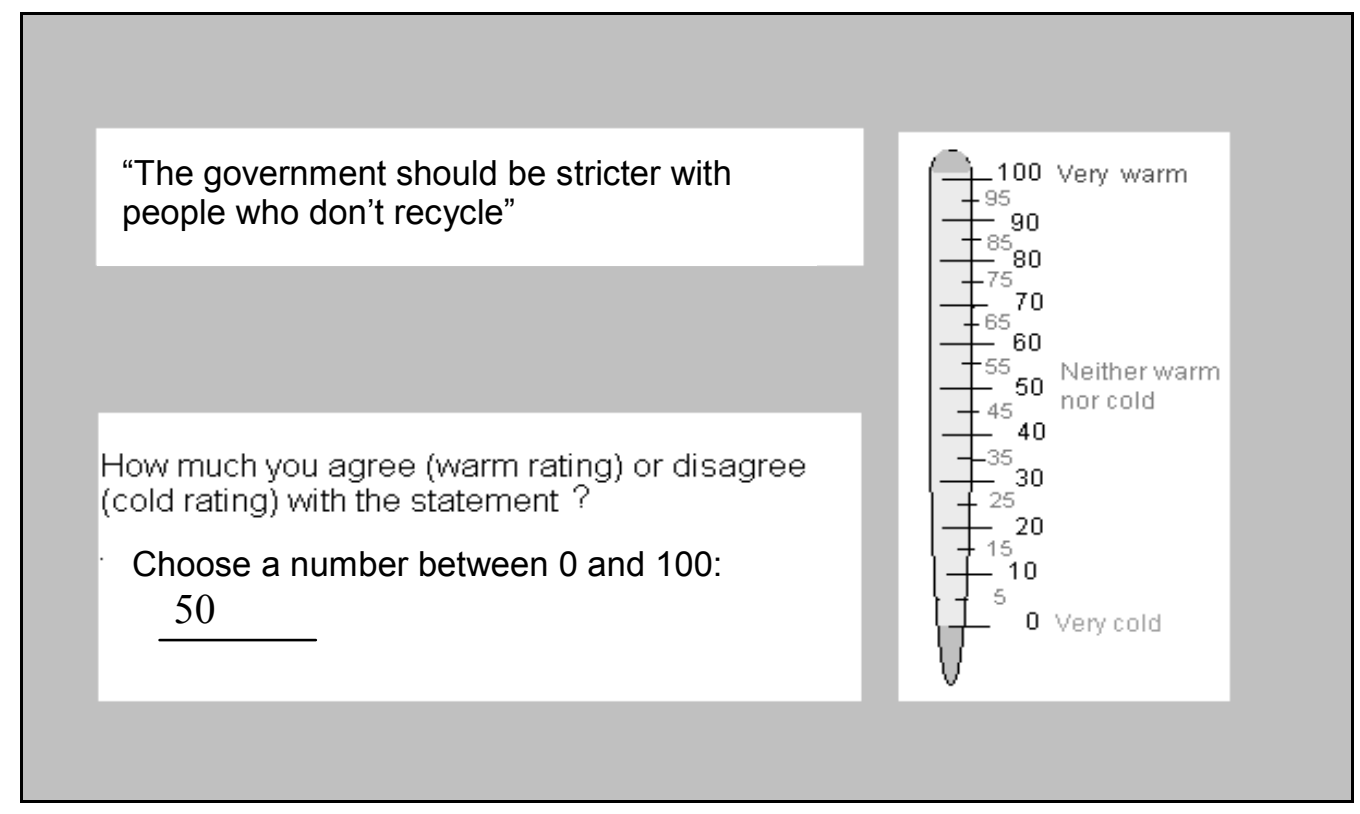


Now please answer the following:

How much do you agree (warm rating) or disagree (cold rating) with the statement?

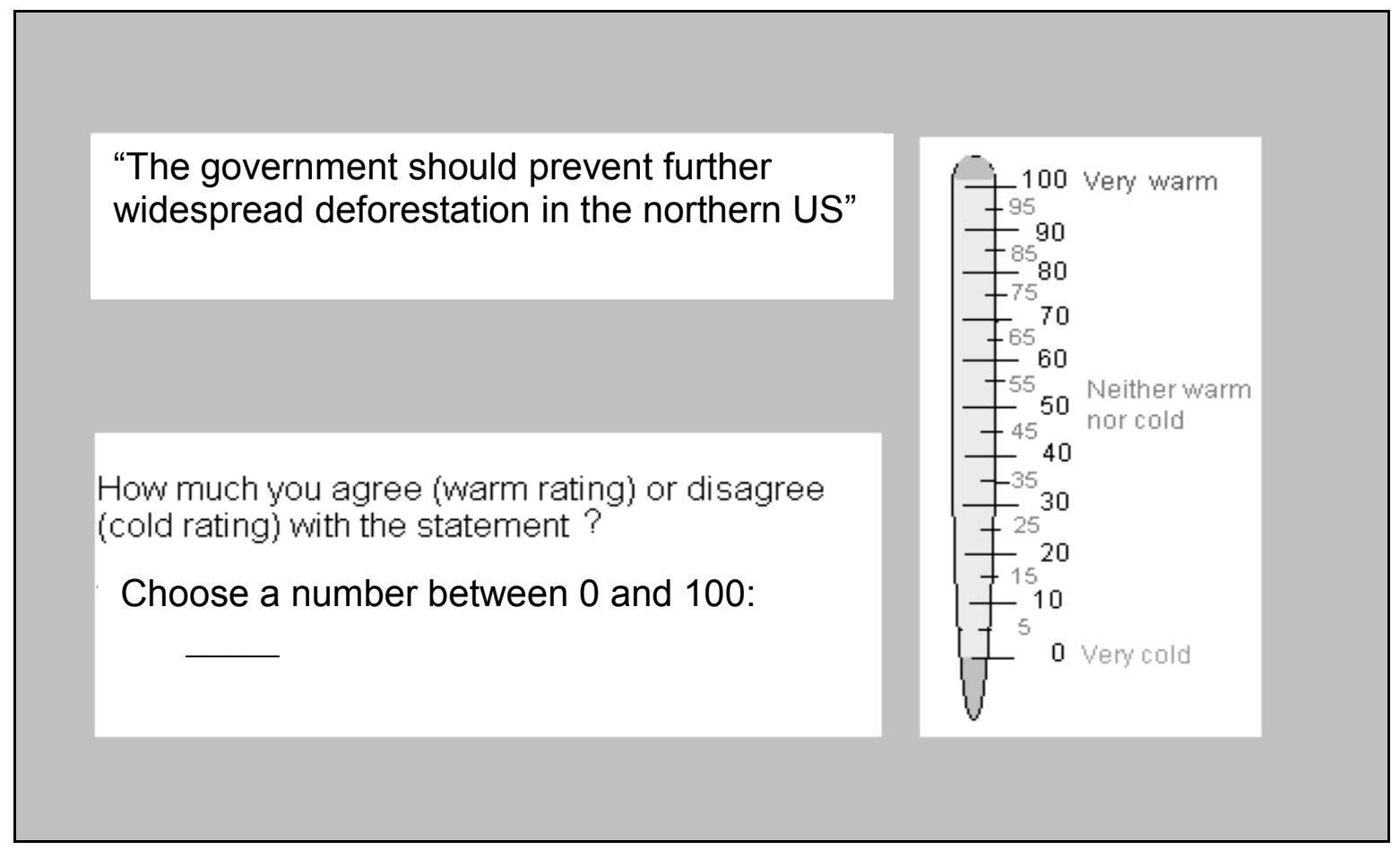

"Anti-smoking programs have been effective in preventing cigarette addiction".

How much you agree (warm rating) or disagree (cold rating) with the statement?

Choose a number between 0 and 100:

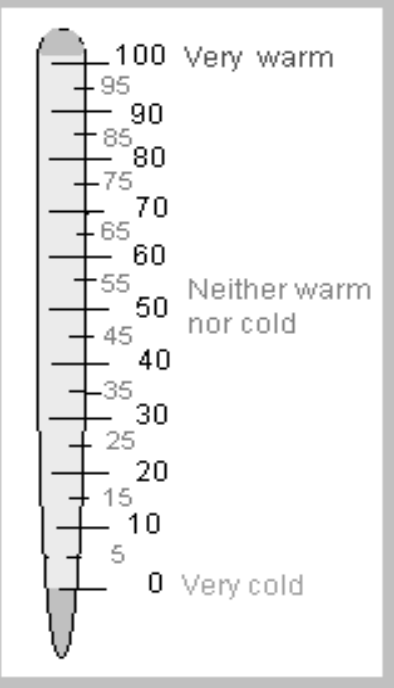


"Government should impose controls on industry to help minimize the effect of acid rain in US".

How much you agree (warm rating) or disagree (cold rating) with the statement?

Choose a number between 0 and 100:

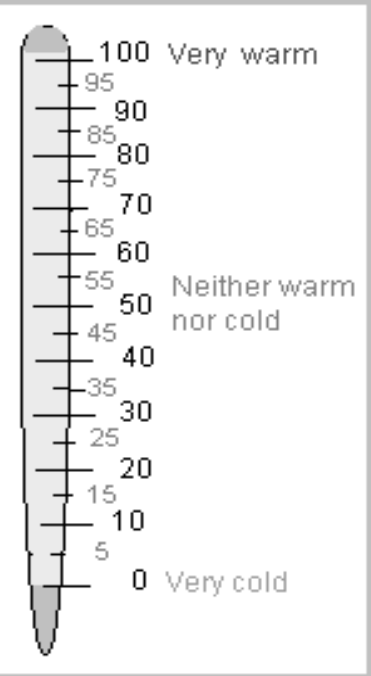

"Oil drilling off the US coast should be increased so that we are not so dependent of foreign oil".

How much you agree (warm rating) or disagree (cold rating) with the statement?

Choose a number between 0 and 100:

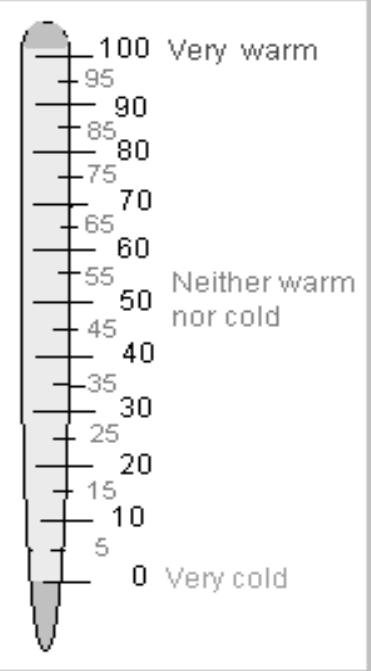




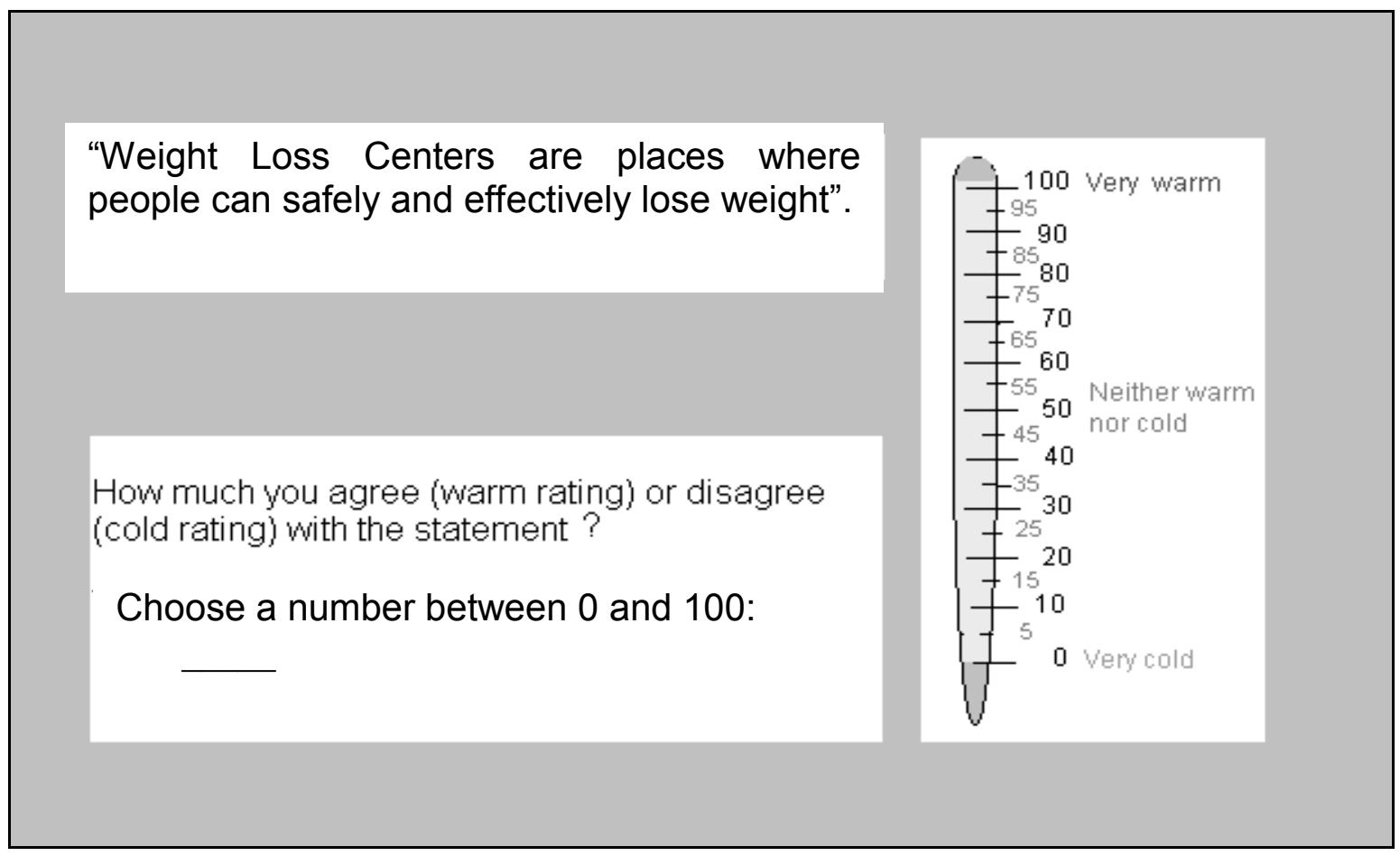

"Smog checks on automobiles have been effective in reducing air pollution".

How much you agree (warm rating) or disagree (cold rating) with the statement?

Choose a number between 0 and 100:

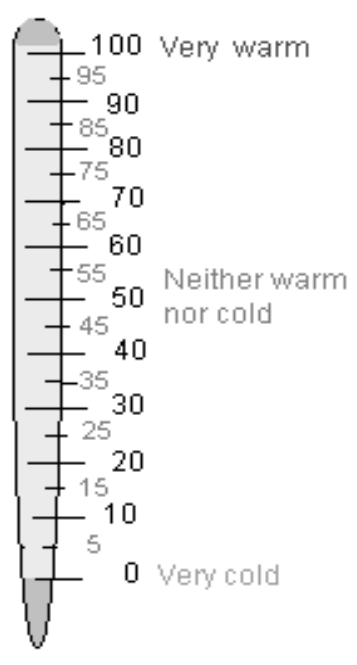


How much you agree (warm rating) or disagree (cold rating) with the statement?

Choose a number between 0 and 100 :

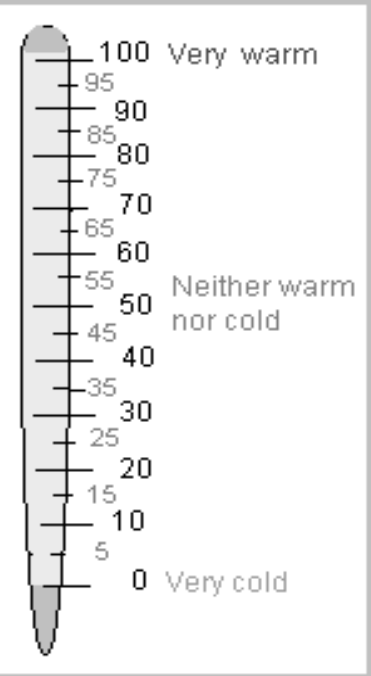

"I am used to reading newspaper articles in crowded and noisy environments".

How much you agree (warm rating) or disagree (cold rating) with the statement?

Choose a number between 0 and 100:

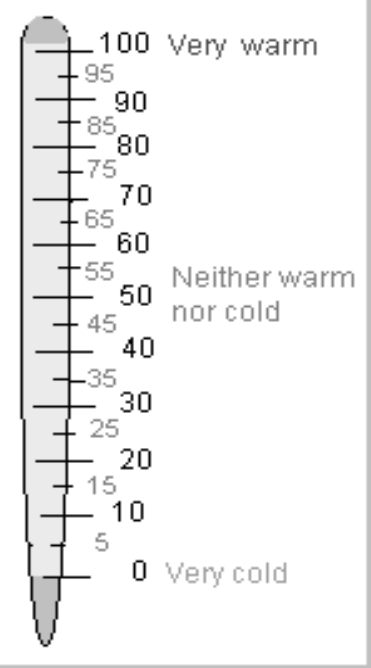


"I am used to studying in crowded and noisy environments".

How much you agree (warm rating) or disagree (cold rating) with the statement?

Choose a number between 0 and 100:

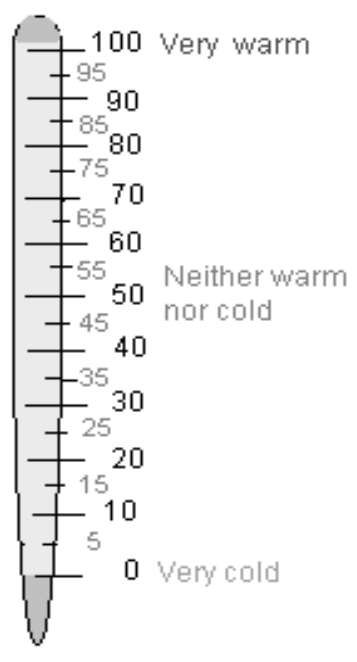




\section{Appendix C: Self-report Questionnaire - Experiment 1 and 3}

Please indicate your answers to the following questions/statements by circling the appropriate number:

How do you feel right now?

\begin{tabular}{|c|c|c|c|c|c|c|}
\hline 1 & 2 & 3 & 4 & 5 & 6 & 7 \\
\hline $\mathrm{Sad}$ & & & & & & Happy \\
\hline
\end{tabular}

How do you feel right now?

\begin{tabular}{|c|c|c|c|c|c|c|}
\hline 1 & 2 & 3 & 4 & 5 & 6 & 7 \\
\hline Discontent & & & & & & Content \\
\hline
\end{tabular}

How would you describe your mood at this time right now?

\begin{tabular}{|c|c|c|c|c|c|c|}
\hline 1 & 2 & 3 & 4 & 5 & 6 & 7 \\
\hline $\mathrm{Bad}$ & & & & & & Good \\
\hline
\end{tabular}

In general, I think I had sufficient capacity to deal with what was demand by the task.

\begin{tabular}{|c|c|c|c|c|c|c|}
\hline 1 & 2 & 3 & 4 & 5 & 6 & 7 \\
\hline $\begin{array}{c}\text { strongly } \\
\text { disagree }\end{array}$ & & & & & & $\begin{array}{c}\text { strongly } \\
\text { agree }\end{array}$ \\
\hline
\end{tabular}

I found this task easy to do.

\begin{tabular}{|c|c|c|c|c|c|c|}
\hline 1 & 2 & 3 & 4 & 5 & 6 & 7 \\
\hline $\begin{array}{c}\text { strongly } \\
\text { disagree }\end{array}$ & & & & & & $\begin{array}{c}\text { strongly } \\
\text { agree }\end{array}$ \\
\hline
\end{tabular}

During my performance I was thinking in other things.

\begin{tabular}{|c|c|c|c|c|c|c|}
\hline 1 & 2 & 3 & 4 & 5 & 6 & 7 \\
\hline $\begin{array}{c}\text { strongly } \\
\text { disagree }\end{array}$ & & & & & & $\begin{array}{c}\text { strongly } \\
\text { agree }\end{array}$ \\
\hline
\end{tabular}

I felt involved in this study.

\begin{tabular}{|c|c|c|c|c|c|c|}
\hline 1 & 2 & 3 & 4 & 5 & 6 & 7 \\
\hline $\begin{array}{c}\text { strongly } \\
\text { disagree }\end{array}$ & & & & & & $\begin{array}{c}\text { strongly } \\
\text { agree }\end{array}$ \\
\hline
\end{tabular}

I tried to answer the questions as more accurate as possible.

\begin{tabular}{|c|c|c|c|c|c|c|}
\hline 1 & 2 & 3 & 4 & 5 & 6 & 7 \\
\hline $\begin{array}{c}\text { strongly } \\
\text { disagree }\end{array}$ & & & & & & $\begin{array}{c}\text { strongly } \\
\text { agree }\end{array}$ \\
\hline
\end{tabular}

I think my answers were those that the experimenter wanted.

\begin{tabular}{|c|c|c|c|c|c|c|}
\hline 1 & 2 & 3 & 4 & 5 & 6 & 7 \\
\hline $\begin{array}{c}\text { strongly } \\
\text { disagree }\end{array}$ & & & & & & $\begin{array}{c}\text { strongly } \\
\text { agree }\end{array}$ \\
\hline
\end{tabular}


I tried to give adequate answers.

\begin{tabular}{|c|c|c|c|c|c|c|}
\hline 1 & 2 & 3 & 4 & 5 & 6 & 7 \\
\hline $\begin{array}{c}\text { strongly } \\
\text { disagree }\end{array}$ & & & & & $\begin{array}{c}\text { strongly } \\
\text { agree }\end{array}$ \\
\hline
\end{tabular}

The presentation of the audio message made me feel the second written message as familiar.

\begin{tabular}{|c|c|c|c|c|c|c|}
\hline 1 & 2 & 3 & 4 & 5 & 6 & 7 \\
\hline $\begin{array}{c}\text { strongly } \\
\text { disagree }\end{array}$ & & & & & & $\begin{array}{c}\text { strongly } \\
\text { agree }\end{array}$ \\
\hline
\end{tabular}

It was easier to answer the questions related to the second written message.

\begin{tabular}{|c|c|c|c|c|c|c|}
\hline 1 & 2 & 3 & 4 & 5 & 6 & 7 \\
\hline $\begin{array}{c}\text { strongly } \\
\text { disagree }\end{array}$ & & & & & & $\begin{array}{c}\text { strongly } \\
\text { agree }\end{array}$ \\
\hline
\end{tabular}

Thank you! 


\section{Appendix D: Statistics of Experiment 1}

\section{Effectiveness of the familiarity manipulation.}

Table 1

Between-Subjects Anova (2 x 2)

\begin{tabular}{crrrrr}
\hline & \multicolumn{1}{c}{ SS } & df & \multicolumn{1}{c}{ MS } & \multicolumn{1}{c}{ F } & \multicolumn{1}{c}{$\mathrm{p}$} \\
\hline Corrected Model & $36,250^{\mathrm{a}}$ & 3 & 12,083 & 4,761 &, 006 \\
Intercept & 954,083 & 1 & 954,083 & 375,937 &, 000 \\
Repetition & 30,083 & 1 & 30,083 & 11,854 &, 001 \\
Argument Quality & 4,083 & 1 & 4,083 & 1,609 &, 211 \\
Rep. * Arg. Qual & 2,083 & 1 & 2,083 &, 821 &, 370 \\
Error & 111,667 & 44 & 2,538 & & \\
Total & 1102,000 & 48 & & & \\
Corrected Total & 147,917 & 47 & & & \\
\hline
\end{tabular}

Table 2

Descriptive statistics of the main effect of the Repetition

\begin{tabular}{clc}
\hline Condition & $M$ & $S D$ \\
\hline No-Repetition & 3,667 &, 325 \\
Repetition & 5,250 &, 325 \\
\hline
\end{tabular}

\section{Atitude-Change Data: Weight Loss Centre Message}

Table 3

Factor Analysis and Reliability

\begin{tabular}{lrrrr}
\hline \multicolumn{4}{c}{ Loadings } \\
\cline { 2 - 5 } & Loading & \% of Variance & Cumulative \% & \multicolumn{1}{c}{$\alpha$} \\
\hline 1 & 1,510 & 50,349 & 50,349 &, 41 \\
2 &, 908 & 30,281 & 80,630 & \\
3 &, 581 & 19,370 & 100,000 & \\
\hline
\end{tabular}

Extraction Method: Principal Component Analysis.

1 components extracted. 
Table 4

Between-Subjects Anova (2 $x$ 2)

\begin{tabular}{crrrrr}
\hline & \multicolumn{1}{c}{ SS } & df & \multicolumn{1}{c}{ MS } & \multicolumn{1}{c}{ F } & \multicolumn{1}{c}{$\mathrm{p}$} \\
\hline Corrected Model & $5,112^{\mathrm{a}}$ & 3 & 1,704 & 1,411 &, 252 \\
Intercept & 65,326 & 1 & 65,326 & 54,108 &, 000 \\
Repetition &, 037 & 1 &, 037 &, 031 &, 862 \\
Argument Quality & 4,484 & 1 & 4,484 & 3,714 &, 060 \\
Rep. * Arg. Qual &, 592 & 1 &, 592 &, 490 &, 488 \\
Error & 53,122 & 44 & 1,207 & & \\
Total & 123,560 & 48 & & & \\
Corrected Total & 58,234 & 47 & & & \\
\hline
\end{tabular}

Table 5

Descriptive statistics of the main effects of Argument Quality

\begin{tabular}{crr}
\hline Condition & \multicolumn{1}{c}{$M$} & \multicolumn{1}{c}{$S D$} \\
\hline Weak &, 861 &, 224 \\
Strong & 1,472 &, 224 \\
\hline
\end{tabular}

\section{Atitude-Change Data: Acid Rain Message}

Table 6

Factor Analysis and Reliability

\begin{tabular}{lrrrr}
\hline \multicolumn{4}{c}{ Loadings } \\
\cline { 2 - 5 } & Loading & \% of Variance & Cumulative \% & \multicolumn{1}{c}{$\alpha$} \\
\hline 1 & 2,212 & 73,732 & 73,732 &, 82 \\
2 &, 466 & 15,530 & 89,261 & \\
3 &, 322 & 10,739 & 100,000 & \\
\hline
\end{tabular}

Extraction Method: Principal Component Analysis.

1 components extracted.

Table 7

Between-Subjects Anova (2 $x$ 2)

\begin{tabular}{crrrrr}
\hline & \multicolumn{1}{c}{$\mathrm{SS}$} & $\mathrm{df}$ & $\mathrm{MS}$ & $\mathrm{F}$ & $\mathrm{p}$ \\
\hline Corrected Model & $19,232^{\mathrm{a}}$ & 3 & 6,411 & 2,452 &, 076 \\
Intercept & 4,822 & 1 & 4,822 & 1,844 &, 181 \\
Repetition &, 432 & 1 &, 432 &, 165 &, 686 \\
Argument Quality & 5,140 & 1 & 5,140 & 1,966 &, 168 \\
Rep. * Arg. Qual & 13,661 & 1 & 13,661 & 5,225 &, 027
\end{tabular}




\begin{tabular}{cccc} 
Error & 115,034 & 44 & 2,614 \\
Total & 139,088 & 48 & \\
Corrected Total & 134,266 & 47 & \\
\hline
\end{tabular}

Table 8

Descriptive statistics of the Argument Quality x Repetition Interaction

\begin{tabular}{ccccc}
\hline & \multicolumn{2}{c}{ No-repetition } & \multicolumn{2}{c}{ Repetition } \\
\cline { 2 - 5 } Condition & $M$ & $S D$ & $M$ & $S D$ \\
\hline Weak &,- 639 &, 467 &, 618 &, 467 \\
Strong & 1,083 &, 467 &, 206 &, 467 \\
\hline
\end{tabular}

Atitude-Change Data: Number of Thoughts

Table 9

Between-Subjects Anova (2 $\times 2$ 2)

\begin{tabular}{crrrrr}
\hline & \multicolumn{1}{c}{ SS } & df & \multicolumn{1}{c}{ MS } & \multicolumn{1}{c}{ F } & \multicolumn{1}{c}{$\mathrm{p}$} \\
\hline Corrected Model & $2,556^{\mathrm{a}}$ & 3 &, 852 & 1,823 &, 157 \\
Intercept & 164,930 & 1 & 164,930 & 352,857 &, 000 \\
Repetition & 2,145 & 1 & 2,145 & 4,589 &, 038 \\
Argument Qual &, 000 & 1 &, 000 &, 001 &, 975 \\
Fam * Qual &, 411 & 1 &, 411 &, 878 &, 354 \\
Error & 20,566 & 44 &, 467 & & \\
Total & 188,052 & 48 & & & \\
Corrected Total & 23,122 & 47 & & & \\
\hline
\end{tabular}

Table 10

Descriptive statistics of the Main effect of Repetition

\begin{tabular}{ccc}
\hline Condition & $M$ & $S D$ \\
\hline No-Repetition & 2,065 &, 140 \\
Repetition & 1,642 &, 140 \\
\hline
\end{tabular}

\section{Atitude-Change Data: Type of Thoughts}

Table 11

Mixed Anova (2 $x 2 \times 2)$, Type of Thoughts as Repeated Measure.

\begin{tabular}{crrrrr}
\hline & SS & df & MS & \multicolumn{1}{c}{ F } & Sig. \\
\hline TypeThought &, 543 & 1 &, 543 & 4,770 &, 034 \\
TypeThought * Rep & 1,989 & 1 & 1,989 & 17,471 &, 000 \\
TypeThought * Arg. Qual & 1,810 & 1 & 1,810 & 15,905 &, 000 \\
TypeThought * Rep * Arg. Qual &, 494 & 1 &, 494 & 4,340 &, 043 \\
& 5,008 & 44 &, 114 & & \\
\hline Error(TypeofThought) & & & & &
\end{tabular}


Table 12

Descriptive statistics of the Main effect of Type of Thoughts

\begin{tabular}{ccc}
\hline Condition & $M$ & $S D$ \\
\hline Favorable &, 266 &, 027 \\
Unfavorable &, 417 &, 052 \\
\hline
\end{tabular}

Table 13

Descriptive statistics of the TypeThought $x$ Repetition Interaction

\begin{tabular}{ccccc}
\hline & \multicolumn{2}{c}{ No-repetition } & \multicolumn{2}{c}{ Repetition } \\
\cline { 2 - 5 } Condition & $M$ & $S D$ & $M$ & $S D$ \\
\hline Favorable &, 436 &, 039 &, 096 &, 039 \\
Unfavorable &, 299 &, 073 &, 535 &, 073 \\
\hline
\end{tabular}

Table 14

Descriptive statistics of the TypeThought x Argument Quality Interaction

\begin{tabular}{ccccc}
\hline & \multicolumn{2}{c}{ Strong } & \multicolumn{2}{c}{ Weak } \\
\cline { 2 - 5 } Condition & $M$ & $S D$ & $M$ & $S D$ \\
\hline Favorable &, 416 &, 039 &, 117 &, 039 \\
Unfavorable &, 292 &, 073 &, 542 &, 073 \\
\hline
\end{tabular}

\section{Atitude-Change Data: Mediation analyses}

Table 15

Regression analyses in the no-repetition condition

\begin{tabular}{cccccc}
\hline & $\begin{array}{c}\text { Unstandardized } \\
\text { Coefficients }\end{array}$ & $\begin{array}{c}\text { Standardized } \\
\text { Coefficients }\end{array}$ & $t$ & Sig. \\
\cline { 2 - 6 } Predictor* & $B$ & $S D$ & & & \\
\hline Arg. Quality & 1,721 &, 522 &, 575 & 3,300 &, 003 \\
Arg. Quality ** &, 740 &, 611 &, 256 & 1,212 &, 241 \\
Thought Index &, 949 &, 373 &, 537 & 2,544 &, 020 \\
\hline
\end{tabular}

* Attitude-Change towards Acid Rain as Dependent Measure

** Thought Index constant

Table 16

Regression analyses in the repetition condition

\begin{tabular}{cccccc}
\hline & \multicolumn{2}{c}{$\begin{array}{c}\text { Unstandardized } \\
\text { Coefficients }\end{array}$} & $\begin{array}{c}\text { Standardized } \\
\text { Coefficients }\end{array}$ & $t$ & Sig. \\
\cline { 2 - 6 } Predictor* & $B$ & $S D$ & & & \\
\hline Arg. Quality &,- 412 &, 774 &,- 113 &,- 533 &, 600 \\
Arg. Quality ** &, 048 &, 944 &, 013 &, 051 &, 960 \\
Thought Index &,- 377 &, 595 &,- 168 &,- 633 &, 534 \\
\hline
\end{tabular}

* Attitude-Change towards Acid Rain as Dependent Measure

** Thought Index constant 


\section{Challenge and Threat Data: Baseline Differences}

Table 17

Between-Subjects Manova (2 x 2)

\begin{tabular}{crrrrr}
\hline & Value* & F & $\begin{array}{r}\text { Hypothesis } \\
\text { df }\end{array}$ & Error df & Sig. \\
\hline Intercept &, 001 & $13643,398^{\mathrm{a}}$ & 4,000 & 39,000 &, 000 \\
Repetition &, 928 &, $751^{\mathrm{a}}$ & 4,000 & 39,000 &, 563 \\
Arg. Quality &, 939 &, $637^{\mathrm{a}}$ & 4,000 & 39,000 &, 640 \\
Rep. * Arg. Qual. &, 953 &, $486^{\mathrm{a}}$ & 4,000 & 39,000 &, 746 \\
\hline
\end{tabular}

*Wilks' Lambda.

\section{Challenge and Threat Data: Task-Engagement}

Table 18

T-tests for hr and ve against zero.

\begin{tabular}{|c|c|c|c|c|c|c|c|}
\hline \multirow[b]{2}{*}{ Condition } & \multirow[b]{2}{*}{$M$} & \multirow[b]{2}{*}{$S D$} & \multirow[b]{2}{*}{$\begin{array}{c}\text { Mean } \\
\text { Difference }\end{array}$} & \multirow[b]{2}{*}{$t(47)$} & \multirow[b]{2}{*}{$p$} & \multicolumn{2}{|c|}{ CI 95\% } \\
\hline & & & & & & $L L$ & $U L$ \\
\hline hr_reactivity & 15,088 & 42,160 & 15,088 & 2,480 & ,017 & 2,846 & 27,331 \\
\hline vc_reactivity & 10,599 & 14,896 & 10,599 & 4,930 &, 000 & 6,274 & 14,925 \\
\hline
\end{tabular}

$\mathrm{CI}=$ Confidence interval $(95 \%) ; \mathrm{LL}=$ lower limit; $\mathrm{UL}=$ upper limit

\section{Challenge and Threat Indexes}

Table 19

Between-Subjects Anova (2 x 2), CO as dependent measure

\begin{tabular}{crrrrr}
\hline & \multicolumn{1}{c}{ SS } & df & \multicolumn{1}{c}{ MS } & \multicolumn{1}{c}{ F } & \multicolumn{1}{c}{$\mathrm{p}$} \\
\hline Corrected Model & $90,636^{\mathrm{a}}$ & 3 & 30,212 & 2,132 &, 110 \\
Intercept & 3,123 & 1 & 3,123 &, 220 &, 641 \\
Repetition & 78,056 & 1 & 78,056 & 5,508 &, 023 \\
Argument Qual & 10,876 & 1 & 10,876 &, 768 &, 386 \\
Fam * Qual & 1,703 & 1 & 1,703 &, 120 &, 730 \\
Error & 623,520 & 44 & 14,171 & & \\
Total & 717,278 & 48 & & & \\
Corrected Total & 714,156 & 47 & & & \\
\hline
\end{tabular}

Table 20

Descriptive statistics of the Main effect of Repetition

\begin{tabular}{crc}
\hline Condition & $M$ & $S D$ \\
\hline No-Repetition & $-1,020$ &, 768 \\
Repetition & 1,530 &, 768 \\
\hline
\end{tabular}


Table 21

Between-Subjects Anova (2 $x$ 2), TPR as dependent measure

\begin{tabular}{crrrrr}
\hline & \multicolumn{1}{c}{ SS } & df & \multicolumn{1}{c}{ MS } & \multicolumn{1}{c}{ F } & \multicolumn{1}{c}{$\mathrm{p}$} \\
\hline Corrected Model & $830928,663^{\mathrm{a}}$ & 3 & 276976,221 & 1,577 &, 209 \\
Intercept & 1138986,228 & 1 & 1138986,228 & 6,483 &, 014 \\
Repetition & 706930,860 & 1 & 706930,860 & 4,024 &, 051 \\
Argument Qual & 12193,036 & 1 & 12193,036 &, 069 &, 793 \\
Fam * Qual & 111804,767 & 1 & 111804,767 &, 636 &, 429 \\
Error & 7730199,007 & 44 & 175686,341 & & \\
Total & 9700113,898 & 48 & & & \\
Corrected Total & 8561127,670 & 47 & & & \\
\hline
\end{tabular}

Table 22

Descriptive statistics of the Main effect of Repetition

\begin{tabular}{crr}
\hline Condition & \multicolumn{1}{c}{$M$} & \multicolumn{1}{c}{$S D$} \\
\hline No-Repetition & 275,400 & 85,559 \\
Repetition & 32,684 & 85,559 \\
\hline
\end{tabular}

Mediation of Unitary Index on Attitude-Change towards Acid Rain

Table 23

Ancova $(2 \times 2)$

\begin{tabular}{crrrrr}
\hline & \multicolumn{1}{c}{ SS } & df & MS & \multicolumn{1}{c}{ F } & \multicolumn{1}{c}{$\mathrm{p}$} \\
\hline Corrected Model & $19,764^{\mathrm{a}}$ & 4 & 4,941 & 1,856 &, 136 \\
Intercept & 4,822 & 1 & 4,822 & 1,811 &, 185 \\
Index_CT &, 532 & 1 &, 532 &, 200 &, 657 \\
Repetition &, 487 & 1 &, 487 &, 183 &, 671 \\
Arg. Quality & 5,451 & 1 & 5,451 & 2,047 &, 160 \\
Rep. * Arg. Qual. & 14,188 & 1 & 14,188 & 5,328 &, 026 \\
Error & 114,502 & 43 & 2,663 & & \\
Total & 139,088 & 48 & & & \\
Corrected Total & 134,266 & 47 & & & \\
\hline
\end{tabular}

Mediation of Unitary Index on Number of Thoughts

Table 24

Ancova (2 $\times 2)$

\begin{tabular}{crrrrr}
\hline & \multicolumn{1}{c}{ SS } & df & \multicolumn{1}{c}{ MS } & \multicolumn{1}{c}{ F } & \multicolumn{1}{c}{$\mathrm{p}$} \\
\hline Corrected Model & $2,827^{\text {a }}$ & 4 &, 707 & 1,497 &, 220 \\
Intercept & 164,930 & 1 & 164,930 & 349,441 &, 000 \\
Index_CT &, 271 & 1 &, 271 &, 574 &, 453 \\
Repetition & 2,226 & 1 & 2,226 & 4,715 &, 035 \\
Arg. Quality &, 006 & 1 &, 006 &, 013 &, 908
\end{tabular}




\begin{tabular}{crrrrr} 
Rep. * Arg. Qual. &, 267 & 1 &, 267 &, 565 &, 456 \\
Error & 20,295 & 43 &, 472 & & \\
Total & 188,052 & 48 & & \\
Corrected Total & 23,122 & 47 & & \\
\hline
\end{tabular}

Mediation of Cognitive evaluations on Attitude-Change towards Acid Rain

Table 25

Ancova $(2 \times 2)$

\begin{tabular}{crrrrr}
\hline & \multicolumn{1}{c}{ SS } & df & \multicolumn{1}{c}{ MS } & \multicolumn{1}{c}{ F } & \multicolumn{1}{c}{$\mathrm{p}$} \\
\hline Corrected Model & $21,246^{\text {a }}$ & 4 & 5,312 & 2,021 &, 108 \\
Intercept & 3,412 & 1 & 3,412 & 1,298 &, 261 \\
Cog Evaluation & 2,014 & 1 & 2,014 &, 766 &, 386 \\
Repetition &, 653 & 1 &, 653 &, 248 &, 621 \\
Arg. Quality & 5,370 & 1 & 5,370 & 2,043 &, 160 \\
Rep. * Arg. Qual. & 14,917 & 1 & 14,917 & 5,676 &, 022 \\
Error & 113,020 & 43 & 2,628 & & \\
Total & 139,088 & 48 & & & \\
Corrected Total & 134,266 & 47 & & & \\
\hline
\end{tabular}

\section{Mediation of Mood on Attitude-Change towards Acid Rain}

Table 26

Ancova $(2 \times 2)$

\begin{tabular}{crrrrr}
\hline & \multicolumn{1}{c}{ SS } & df & \multicolumn{1}{c}{ MS } & \multicolumn{1}{l}{ F } & \multicolumn{1}{l}{ p } \\
\hline Corrected Model & $22,355^{\mathrm{a}}$ & 4 & 5,589 & 2,147 &, 091 \\
Intercept & 4,645 & 1 & 4,645 & 1,785 &, 189 \\
Mood & 3,123 & 1 & 3,123 & 1,200 &, 279 \\
Repetition &, 545 & 1 &, 545 &, 209 &, 650 \\
Arg. Quality & 5,402 & 1 & 5,402 & 2,076 &, 157 \\
Rep. * Arg. Qual. & 12,849 & 1 & 12,849 & 4,937 &, 032 \\
Error & 111,911 & 43 & 2,603 & & \\
Total & 139,088 & 48 & & & \\
Corrected Total & 134,266 & 47 & & & \\
\hline
\end{tabular}


Appendix E: Material by Condition (Experiment 2)

Examples of non-demanding/challenge trials (difference between shapes 1,5 cm).

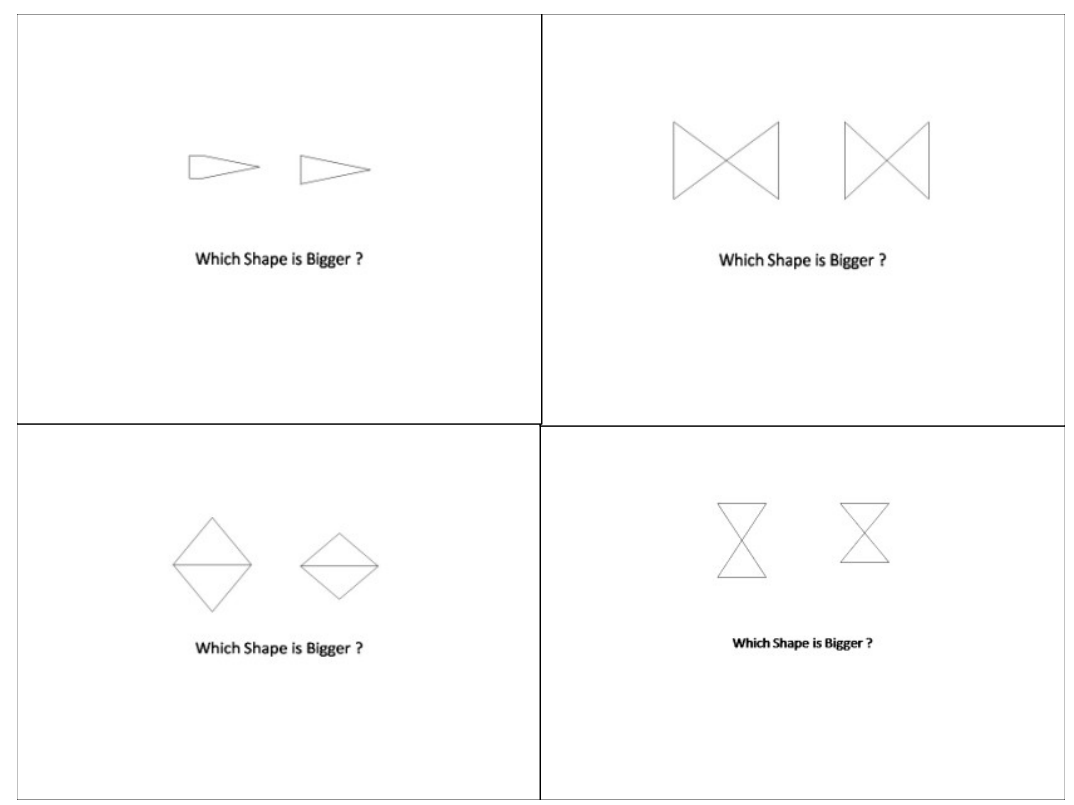

Examples of demanding/threat trials (difference between shapes $0,5 \mathrm{~cm}$ ).

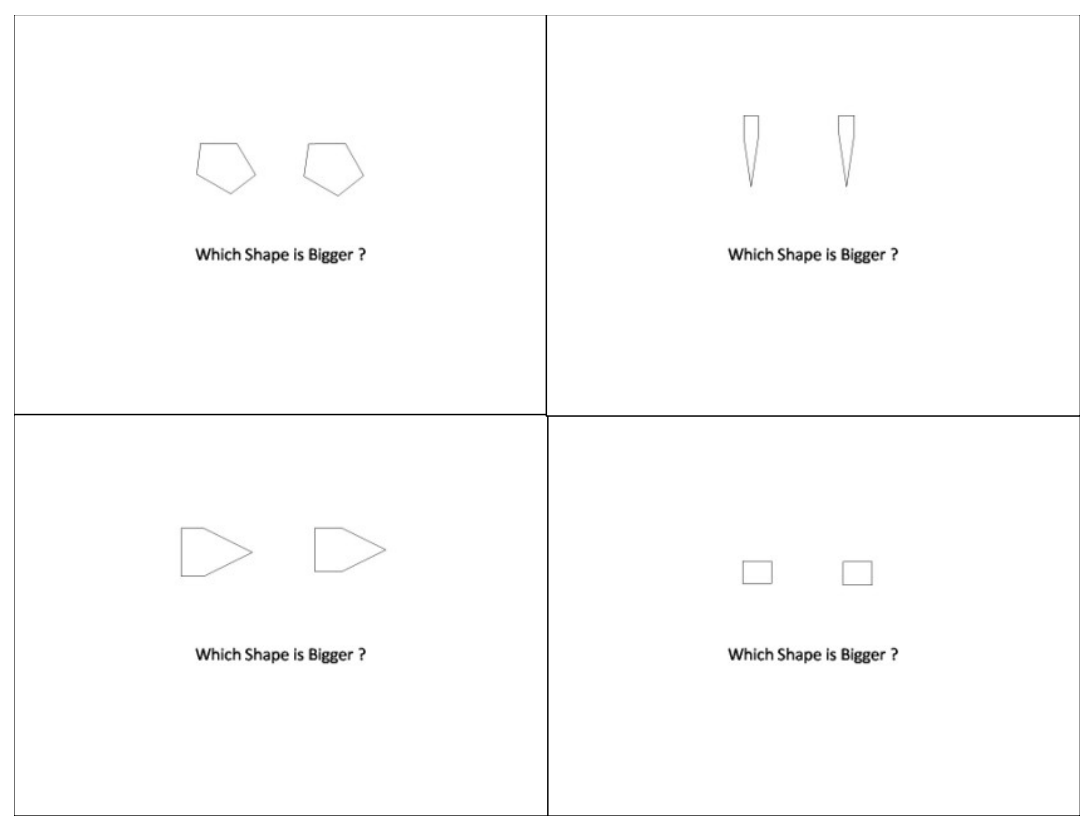


Examples of neutral trials trials (difference between shapes $1 \mathrm{~cm}$ ).

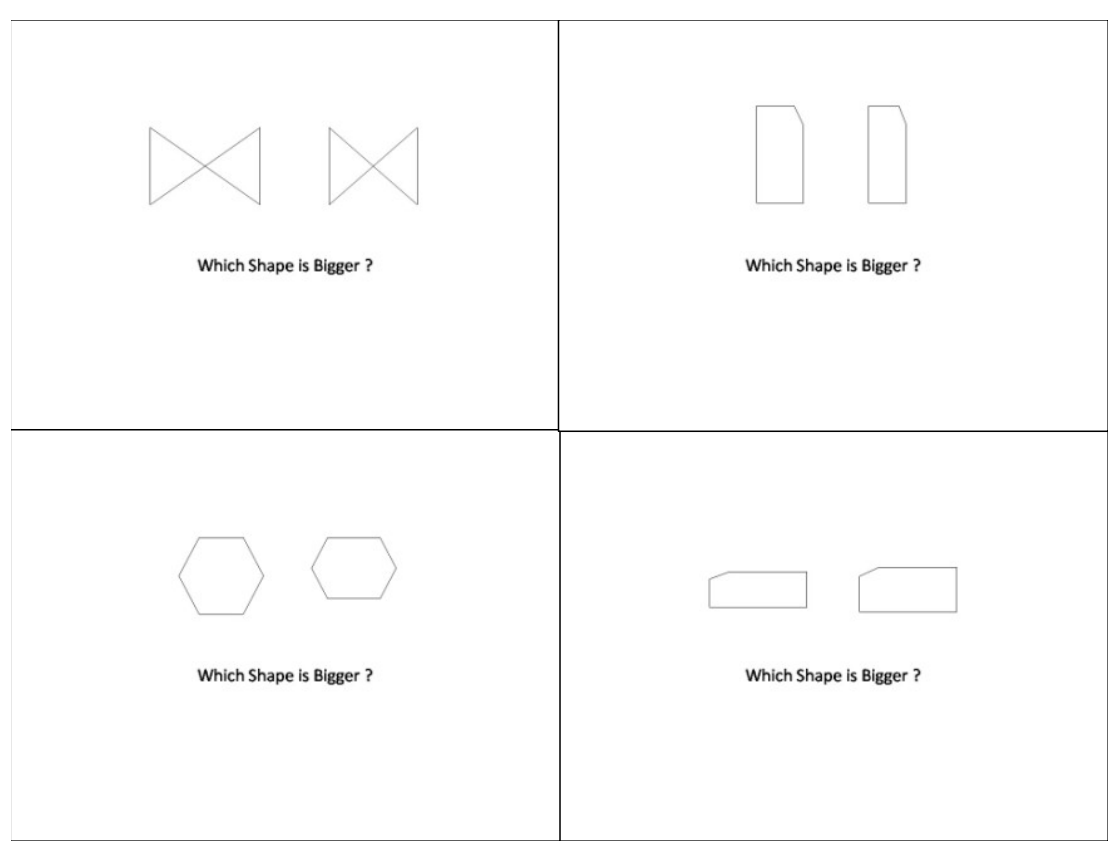




\section{Appendix F: Self Report questionnaire - Experiment 2 and 3}

Please indicate your answers to the following questions/statements by circling the appropriate number:

How do you feel right now?

\begin{tabular}{|c|c|c|c|c|c|c|}
\hline 1 & 2 & 3 & 4 & 5 & 6 & 7 \\
\hline $\mathrm{Sad}$ & & & & & & Happy \\
\hline
\end{tabular}

How do you feel right now?

\begin{tabular}{|c|c|c|c|c|c|c|}
\hline 1 & 2 & 3 & 4 & 5 & 6 & 7 \\
\hline Discontent & & & & & & Content \\
\hline
\end{tabular}

How would you describe your mood at this time right now?

\begin{tabular}{|c|c|c|c|c|c|c|}
\hline 1 & 2 & 3 & 4 & 5 & 6 & 7 \\
\hline $\begin{array}{c}\text { Very } \\
\text { Negative }\end{array}$ & & & & & & $\begin{array}{c}\text { Very } \\
\text { Positive }\end{array}$ \\
\hline
\end{tabular}

I think that Phase 1 was demanding.

\begin{tabular}{|c|c|c|c|c|c|c|}
\hline 1 & 2 & 3 & 4 & 5 & 6 & 7 \\
\hline $\begin{array}{c}\text { strongly } \\
\text { disagree }\end{array}$ & & & & & & $\begin{array}{c}\text { strongly } \\
\text { agree }\end{array}$ \\
\hline
\end{tabular}

In general, I think I had sufficient capacity to deal with what was demanded by the game

\begin{tabular}{|c|c|c|c|c|c|c|}
\hline 1 & 2 & 3 & 4 & 5 & 6 & 7 \\
\hline $\begin{array}{c}\text { strongly } \\
\text { disagree }\end{array}$ & & & & & & $\begin{array}{c}\text { strongly } \\
\text { agree }\end{array}$ \\
\hline
\end{tabular}

On Phase 1, it was difficult to perceive which one of the two shapes was bigger.

\begin{tabular}{|c|c|c|c|c|c|c|}
\hline 1 & 2 & 3 & 4 & 5 & 6 & 7 \\
\hline $\begin{array}{c}\text { strongly } \\
\text { disagree }\end{array}$ & & & & & & $\begin{array}{c}\text { strongly } \\
\text { agree }\end{array}$ \\
\hline
\end{tabular}

During my performance on Phase $1 \mathrm{I}$ was thinking in other things.

\begin{tabular}{|c|c|c|c|c|c|c|}
\hline 1 & 2 & 3 & 4 & 5 & 6 & 7 \\
\hline $\begin{array}{c}\text { strongly } \\
\text { disagree }\end{array}$ & & & & & & $\begin{array}{c}\text { strongly } \\
\text { agree }\end{array}$ \\
\hline
\end{tabular}

I felt involved in Phase 1.

\begin{tabular}{|c|c|c|c|c|c|c|}
\hline 1 & 2 & 3 & 4 & 5 & 6 & 7 \\
\hline $\begin{array}{c}\text { strongly } \\
\text { disagree }\end{array}$ & & & & & & $\begin{array}{c}\text { strongly } \\
\text { agree }\end{array}$ \\
\hline
\end{tabular}

I felt involved in Phase 2.

\begin{tabular}{|l|l|l|l|l|l|l|}
\hline 1 & 2 & 3 & 4 & 5 & 6 & 7 \\
\hline
\end{tabular}




\begin{tabular}{|c|l|l|l|l|l|c|}
\hline $\begin{array}{l}\text { strongly } \\
\text { disagree }\end{array}$ & & & & & $\begin{array}{c}\text { strongly } \\
\text { agree }\end{array}$ \\
\hline
\end{tabular}

I tried to answer the questions on Phase 2 as more accurate as possible.

\begin{tabular}{|c|c|c|c|c|c|c|}
\hline 1 & 2 & 3 & 4 & 5 & 6 & 7 \\
\hline $\begin{array}{c}\text { strongly } \\
\text { disagree }\end{array}$ & & & & & & $\begin{array}{c}\text { strongly } \\
\text { agree }\end{array}$ \\
\hline
\end{tabular}

It was easier to answer the questions related to Phase 2 , than perform the game on phase 1 and 3.

\begin{tabular}{|c|c|c|c|c|c|c|}
\hline 1 & 2 & 3 & 4 & 5 & 6 & 7 \\
\hline $\begin{array}{c}\text { strongly } \\
\text { disagree }\end{array}$ & & & & & & $\begin{array}{c}\text { strongly } \\
\text { agree }\end{array}$ \\
\hline
\end{tabular}

I think my answers on Phase 2 were those that the experimenter wanted.

\begin{tabular}{|c|c|c|c|c|c|c|}
\hline 1 & 2 & 3 & 4 & 5 & 6 & 7 \\
\hline $\begin{array}{c}\text { strongly } \\
\text { disagree }\end{array}$ & & & & & & $\begin{array}{c}\text { strongly } \\
\text { agree }\end{array}$ \\
\hline
\end{tabular}

I tried to give adequate answers.

\begin{tabular}{|c|c|c|c|c|c|c|}
\hline 1 & 2 & 3 & 4 & 5 & 6 & 7 \\
\hline $\begin{array}{c}\text { strongly } \\
\text { disagree }\end{array}$ & & & & & & $\begin{array}{c}\text { strongly } \\
\text { agree }\end{array}$ \\
\hline
\end{tabular}

During my performance on Phase 2 I was thinking in other things.

\begin{tabular}{|c|c|c|c|c|c|c|}
\hline 1 & 2 & 3 & 4 & 5 & 6 & 7 \\
\hline $\begin{array}{c}\text { strongly } \\
\text { disagree }\end{array}$ & & & & & & $\begin{array}{c}\text { strongly } \\
\text { agree }\end{array}$ \\
\hline
\end{tabular}

Thank you! 


\section{Appendix G: Statistics of Experiment 2}

\section{Challenge and Threat Data. Effectiveness of the task-demands manipulation.}

Table 1

Anova one-way.

\begin{tabular}{crrrrr}
\hline & \multicolumn{1}{c}{ SS } & df & \multicolumn{1}{c}{ MS } & \multicolumn{1}{c}{ F } & \multicolumn{1}{c}{$\mathrm{p}$} \\
\hline Corrected Model & $9,680^{\mathrm{a}}$ & 1 & 9,680 & 3,799 &, 057 \\
Intercept & 1352,000 & 1 & 1352,000 & 530,543 &, 000 \\
Demands & 9,680 & 1 & 9,680 & 3,799 &, 057 \\
Error & 122,320 & 48 & 2,548 & & \\
Total & 1484,000 & 50 & & & \\
Corrected Total & 132,000 & 49 & & & \\
\hline
\end{tabular}

Table 2

Descriptive statistics of the main effect of the Demands

\begin{tabular}{ccc}
\hline Condition & $M$ & $S D$ \\
\hline Non-Demanding & 5,640 & 1,629 \\
Demanding & 4,760 & 1,562 \\
\hline
\end{tabular}

\section{Challenge and Threat Data: Baseline Differences}

Table 3

Between-Subjects Manova (2 × 2)

\begin{tabular}{crrrrr}
\hline & Value & F & $\begin{array}{r}\text { Hypothesis } \\
\text { df }\end{array}$ & Error df & Sig. \\
\hline Intercept &, 006 & $1644,924^{\mathrm{a}}$ & 4,000 & 39,000 &, 000 \\
Argument Quality &, 947 &, $549^{\mathrm{a}}$ & 4,000 & 39,000 &, 701 \\
Demands &, 859 & $1,600^{\mathrm{a}}$ & 4,000 & 39,000 &, 194 \\
Arg. Qual x Demands &, 829 & $2,016^{\mathrm{a}}$ & 4,000 & 39,000 &, 111 \\
\hline
\end{tabular}

*Wilks' Lambda.

\section{Challenge and Threat Data: Task-Engagement}

Table 4

T-tests for hr and vc against zero

\begin{tabular}{|c|c|c|c|c|c|c|c|}
\hline \multirow[b]{2}{*}{ Condition } & \multirow[b]{2}{*}{$M$} & \multirow[b]{2}{*}{$S D$} & \multirow[b]{2}{*}{$\begin{array}{c}\text { Mean } \\
\text { Difference }\end{array}$} & \multirow[b]{2}{*}{$t(51)$} & \multirow[b]{2}{*}{$p$} & \multicolumn{2}{|c|}{ CI 95\% } \\
\hline & & & & & & $L L$ & $U L$ \\
\hline hr_reactivity* & 12,768 & 28,515 & 12,768 & 3,229 & ,002 & 4,829 & 20,707 \\
\hline vc_reactivity* & 1,874 & 5,277 & 1,87424 & 2,561 &, 013 & ,404 & 3,343 \\
\hline
\end{tabular}


$\begin{array}{llllllll}\text { hr_reactivity** } & 12,858 & 24,256 & 12,858 & 3,823 & , 000 & 6,105 & 19,611\end{array}$

\begin{tabular}{llllllll} 
vc_reactivity** & 1,195 & 4,358 & 1,195 & 1,978 &, 053 &,- 017 & 2,409 \\
\hline Cl-Confidence
\end{tabular}

$\mathrm{CI}=$ Confidence interval (95\%); $\mathrm{LL}=$ lower limit; $\mathrm{UL}=$ upper limit

*Values relative to the game task

$* *$ Values relative to the persuasion task

\section{Challenge and Threat Indexes}

Table 5

Between-Subjects Anova (2 $\times 2$ 2), CO as dependent measure, relative to the game task.

\begin{tabular}{crrrrr}
\hline & \multicolumn{1}{c}{ SS } & df & \multicolumn{1}{c}{ MS } & \multicolumn{1}{c}{ F } & \multicolumn{1}{c}{$\mathrm{p}$} \\
\hline Corrected Model & $25,985^{\mathrm{a}}$ & 1 & 25,985 & 4,296 &, 043 \\
Intercept &, 383 & 1 &, 383 &, 063 &, 802 \\
Demands & 25,985 & 1 & 25,985 & 4,296 &, 043 \\
Error & 302,400 & 50 & 6,048 & & \\
Total & 328,768 & 52 & & & \\
Corrected Total & 328,385 & 51 & & & \\
\hline
\end{tabular}

Table 6

Descriptive statistics of the Main effect of demands

\begin{tabular}{ccc}
\hline Condition & $M$ & $S D$ \\
\hline Demanding &,- 792 & .49 \\
Non-Demanding &, 621 & .48 \\
\hline
\end{tabular}

Table 7

Between-Subjects Anova (2 $\times 2$ 2), TPR as dependent measure, relative to the game task.

\begin{tabular}{crrrrr}
\hline & \multicolumn{1}{c}{ SS } & df & \multicolumn{1}{c}{ MS } & \multicolumn{1}{c}{ F } & \multicolumn{1}{c}{$\mathrm{p}$} \\
\hline Corrected Model & $372074,584^{\mathrm{a}}$ & 1 & 372074,584 & 5,472 &, 023 \\
Intercept & 27806,694 & 1 & 27806,694 &, 409 &, 525 \\
Demands & 372074,584 & 1 & 372074,584 & 5,472 &, 023 \\
Error & 3399571,319 & 50 & 67991,426 & & \\
Total & 3799452,597 & 52 & & & \\
Corrected Total & 3771645,903 & 51 & & & \\
\hline
\end{tabular}

Table 8

Descriptive statistics of the Main effect of Demands

\begin{tabular}{crc}
\hline Condition & \multicolumn{1}{c}{$M$} & \multicolumn{1}{c}{$S D$} \\
\hline Demanding & 61,464 & 25,60 \\
Non-Demanding & $-107,713$ & 51,14 \\
\hline
\end{tabular}


Table 9

Between-Subjects Anova (2 $\times 2$ 2), CO as dependent measure, relative to the persuasion task.

\begin{tabular}{crrrrr}
\hline & \multicolumn{1}{c}{ SS } & df & \multicolumn{1}{c}{ MS } & \multicolumn{1}{c}{ F } & \multicolumn{1}{c}{$\mathrm{p}$} \\
\hline Corrected Model & $99,902^{\mathrm{a}}$ & 3 & 33,301 & 1,626 &, 196 \\
Intercept &, 073 & 1 &, 073 &, 004 &, 952 \\
Argument Qual & 1,399 & 1 & 1,399 &, 068 &, 795 \\
Demands & 89,219 & 1 & 89,219 & 4,358 &, 042 \\
Qual * Demands & 10,474 & 1 & 10,474 &, 512 &, 478 \\
Error & 982,775 & 48 & 20,474 & & \\
Total & 1082,677 & 52 & & & \\
Corrected Total & 1082,677 & 51 & & & \\
\hline
\end{tabular}

Table 10

Descriptive statistics of the Main effect of Demands

\begin{tabular}{ccc}
\hline Condition & $M$ & $S D$ \\
\hline Demanding & -1.48 &, 92 \\
Non-Demanding & 1.22 &, 88 \\
\hline
\end{tabular}

Table 11

Between-Subjects Anova (2 $x 2$ ), TPR as dependent measure, relative to the persuasion task.

\begin{tabular}{crrrrr}
\hline & \multicolumn{1}{c}{$\mathrm{SS}$} & $\mathrm{df}$ & \multicolumn{1}{c}{ MS } & \multicolumn{1}{c}{$\mathrm{F}$} & \multicolumn{1}{c}{$\mathrm{p}$} \\
\hline Corrected Model & $988690,915^{\mathrm{a}}$ & 3 & 329563,638 & 2,950 &, 042 \\
Intercept & 1,101 & 1 & 1,101 &, 000 &, 998 \\
Argument Qual & 6020,011 & 1 & 6020,011 &, 054 &, 817 \\
Demands & 439016,067 & 1 & 439016,067 & 3,929 &, 053 \\
Qual * Demands & 548961,961 & 1 & 548961,961 & 4,913 &, 031 \\
Error & 5362989,743 & 48 & 111728,953 & & \\
Total & 6354829,080 & 52 & & & \\
Corrected Total & 6351680,658 & 51 & & & \\
\hline
\end{tabular}

Table 12

Descriptive statistics of the Main effect of Demands

\begin{tabular}{ccc}
\hline Condition & $M$ & $S D$ \\
\hline Demanding & 92,011 & 65,748 \\
Non-Demanding & $-92,303$ & 63,224 \\
\hline
\end{tabular}




\section{Attitude-Change Data.}

Table 13

Factor Analysis and Reliability

\begin{tabular}{lrrrr}
\hline \multicolumn{4}{c}{ Loadings } \\
\cline { 2 - 5 } & Loading & \% of Variance & Cumulative \% & \multicolumn{1}{c}{$\alpha$} \\
\hline 1 & 1,944 & 64,811 & 64,811 &, 72 \\
2 &, 724 & 24,126 & 88,937 & \\
3 &, 332 & 11,063 & 100,000 & \\
\hline
\end{tabular}

Extraction Method: Principal Component Analysis.

1 components extracted.

Table 14

Anova (2 $x$ 2)

\begin{tabular}{crrrrr}
\hline & \multicolumn{1}{c}{ SS } & df & \multicolumn{1}{c}{ MS } & \multicolumn{1}{c}{ F } & \multicolumn{1}{c}{$\mathrm{p}$} \\
\hline Corrected Model & $9,346^{\mathrm{a}}$ & 3 & 3,115 & 1,665 &, 187 \\
Intercept & 10,118 & 1 & 10,118 & 5,409 &, 024 \\
Demands &, 857 & 1 &, 857 &, 458 &, 502 \\
Argument Qual &, 682 & 1 &, 682 &, 364 &, 549 \\
Demands * Qual & 7,680 & 1 & 7,680 & 4,105 &, 048 \\
Error & 89,796 & 48 & 1,871 & & \\
Total & 110,729 & 52 & & & \\
Corrected Total & 99,141 & 51 & & & \\
\hline
\end{tabular}

Table 15

Descriptive statistics of the Demands $x$ Argument quality Interaction

\begin{tabular}{ccccc}
\hline & \multicolumn{2}{c}{ Non-Demanding } & \multicolumn{2}{c}{ Demanding } \\
\cline { 2 - 5 } Condition & $M$ & $S D$ & \multicolumn{1}{c}{$M$} & \multicolumn{1}{c}{$S D$} \\
\hline Strong &, 043 &, 395 & 1,071 &, 366 \\
Weak &, 584 &, 366 &, 071 &, 395 \\
\hline
\end{tabular}

Table 16

Contrasts revealing the impact of the version of the game/motivational state on the differentiation between strong and weak arguments in attitude-change.

\begin{tabular}{cccccccccc}
\hline & \multicolumn{2}{c}{ Strong Arguments } & & \multicolumn{2}{c}{ Weak Arguments } & & & \multicolumn{2}{c}{$C I 95 \%$} \\
\cline { 2 - 3 } \cline { 8 - 10 } Condition & $M$ & $S D$ & & $M$ & $S D$ & $t(48)$ & $p$ & $L L$ & $U L$ \\
\hline Demanding & 1.08 & 1.01 & & .08 & .51 & 1.81 & .07 & -.08 & 2.08 \\
\hline Non-Demanding & .02 & 1.42 & & .52 & .56 & 1.06 & .29 & -1.62 & 0.54 \\
\hline
\end{tabular}

$\mathrm{CI}=$ Confidence interval $(95 \%) ; \mathrm{LL}=$ lower limit; $\mathrm{UL}=$ upper limit 
Mediation of the Unitary Index on Attitude-Change towards Acid Rain

Table 17

Ancova (2 $\times 2)$

\begin{tabular}{crrrrr}
\hline & \multicolumn{1}{c}{ SS } & df & \multicolumn{1}{c}{ MS } & \multicolumn{1}{c}{ F } & \multicolumn{1}{c}{$\mathrm{p}$} \\
\hline Corrected Model & $10,472^{\mathrm{a}}$ & 4 & 2,618 & 1,388 &, 253 \\
Intercept & 10,000 & 1 & 10,000 & 5,301 &, 026 \\
Index_CT & 1,127 & 1 & 1,127 &, 597 &, 443 \\
Demands & 1,438 & 1 & 1,438 &, 762 &, 387 \\
Arg. Quality &, 616 & 1 &, 616 &, 327 &, 570 \\
Demands * Qual. & 8,617 & 1 & 8,617 & 4,568 &, 038 \\
Error & 88,669 & 47 & 1,887 & & \\
Total & 110,729 & 52 & & & \\
Corrected Total & 99,141 & 51 & & & \\
\hline
\end{tabular}

Mediation of Cognitive evaluations on Attitude-Change towards Acid Rain

Table 18

Ancova $(2 \times 2)$

\begin{tabular}{crrrrr}
\hline & \multicolumn{1}{c}{ SS } & df & \multicolumn{1}{c}{ MS } & \multicolumn{1}{c}{ F } & \multicolumn{1}{c}{$\mathrm{p}$} \\
\hline Corrected Model & $10,746^{\text {a }}$ & 4 & 2,687 & 1,407 &, 247 \\
Intercept &, 314 & 1 &, 314 &, 165 &, 687 \\
Cog. Evaluations &, 053 & 1 &, 053 &, 028 &, 869 \\
Demands &, 546 & 1 &, 546 &, 286 &, 595 \\
Argument Quality & 1,428 & 1 & 1,428 &, 748 &, 392 \\
Demands * Arg. Qual & 8,105 & 1 & 8,105 & 4,246 &, 045 \\
Error & 85,902 & 45 & 1,909 & & \\
Total & 105,905 & 50 & & & \\
Corrected Total & 96,648 & 49 & & & \\
\hline
\end{tabular}

\section{Mediation of Mood on Attitude-Change towards Acid Rain}

Table 19

Ancova $(2 \times 2)$

\begin{tabular}{crrrrr}
\hline & SS & df & \multicolumn{1}{c}{ MS } & \multicolumn{1}{c}{ F } & \multicolumn{1}{c}{$\mathrm{p}$} \\
\hline Corrected Model & $10,926^{\mathrm{a}}$ & 4 & 2,732 & 1,434 &, 238 \\
Intercept &, 865 & 1 &, 865 &, 454 &, 504 \\
Mood &, 233 & 1 &, 233 &, 122 &, 728 \\
Arg. Quality & 1,499 & 1 & 1,499 &, 787 &, 380 \\
Demands &, 478 & 1 &, 478 &, 251 &, 619 \\
Qual. * Demands & 8,377 & 1 & 8,377 & 4,398 &, 042
\end{tabular}




\begin{tabular}{lrrr} 
Error & 85,722 & 45 & 1,905 \\
Total & 105,905 & 50 & \\
rected Total & 96,648 & 49 & \\
\hline
\end{tabular}




\section{Appendix H: Statistics of Experiment 3}

\section{Challenge and Threat Data. Effectiveness of the task-demands manipulation.}

Table 1

Anova (2 $x$ 2).

\begin{tabular}{crrrrr}
\hline & \multicolumn{1}{c}{$\mathrm{SS}$} & $\mathrm{df}$ & \multicolumn{1}{c}{ MS } & \multicolumn{1}{c}{$\mathrm{F}$} & \multicolumn{1}{c}{$\mathrm{p}$} \\
\hline Corrected Model & $33,107^{\mathrm{a}}$ & 3 & 11,036 & 5,563 &, 002 \\
Intercept & 2914,588 & 1 & 2914,588 & 1469,294 &, 000 \\
Presence &, 766 & 1 &, 766 &, 386 &, 536 \\
Demands & 31,972 & 1 & 31,972 & 16,118 &, 000 \\
Presence * Demands &, 866 & 1 &, 866 &, 436 &, 510 \\
Error & 180,514 & 91 & 1,984 & & \\
Total & 3126,000 & 95 & & & \\
Corrected Total & 213,621 & 94 & & & \\
\hline
\end{tabular}

Table 2

Descriptive statistics of the main effect of the Demands

\begin{tabular}{clc}
\hline Condition & $M$ & $S D$ \\
\hline Non-Demanding & 6,131 &, 208 \\
Demanding & 4,968 &, 202 \\
\hline
\end{tabular}

\section{Challenge and Threat Data: Baseline Differences}

Table 3

Between-Subjects Manova $(2 \times 2 \times 2)$

\begin{tabular}{crrrrr}
\hline & Value* & F & $\begin{array}{c}\text { Hypothesis } \\
\text { df }\end{array}$ & Error df & Sig. \\
\hline Intercept &, 010 & 2177,496 & 4,000 & 86,000 &, 000 \\
Presence &, 933 & 1,537 & 4,000 & 86,000 &, 199 \\
Demands &, 986 &, 310 & 4,000 & 86,000 &, 871 \\
Argument Quality &, 919 & 1,889 & 4,000 & 86,000 &, 120 \\
Presence * Demands &, 943 & 1,309 & 4,000 & 86,000 &, 273 \\
Presence * Arg. Quality &, 968 &, 710 & 4,000 & 86,000 &, 587 \\
Demands * Arg. Qual &, 972 &, 616 & 4,000 & 86,000 &, 653 \\
Pres. * Demand * Arg. Qual &, 994 &, 139 & 4,000 & 86,000 &, 967 \\
\hline *Wilks' Lambda. & & & & &
\end{tabular}




\section{Challenge and Threat Data: Task-Engagement}

Table 4

T-tests for hr and vc against zero, for the presence condition

\begin{tabular}{|c|c|c|c|c|c|c|c|}
\hline \multirow[b]{2}{*}{ Condition } & \multirow[b]{2}{*}{$\bar{M}$} & \multirow[b]{2}{*}{$S D$} & \multirow[b]{2}{*}{$\begin{array}{c}\text { Mean } \\
\text { Difference }\end{array}$} & \multirow[b]{2}{*}{$t(49)$} & \multirow[b]{2}{*}{$p$} & \multicolumn{2}{|c|}{ CI 95\% } \\
\hline & & & & & & $L L$ & $U L$ \\
\hline hr_reactivity* & 10,238 & 22,886 & 10,238 & 3,163 & ,003 & 3,733 & 16,742 \\
\hline vc_reactivity* & 6,520 & 23,870 & 6,520 & 1,931 &, 059 &,- 264 & 13,304 \\
\hline hr_reactivity** & 5,928 & 17,879 & 5,928 & 2,345 & ,023 & ,847 & 11,009 \\
\hline vc_reactivity** & 7,326 & 24,152 & 7,326 & 2,145 &, 037 & ,462 & 14,191 \\
\hline
\end{tabular}

Table 5

T-tests for hr and vc against zero, for the alone condition

\begin{tabular}{|c|c|c|c|c|c|c|c|}
\hline \multirow[b]{2}{*}{ Condition } & \multirow[b]{2}{*}{$\bar{M}$} & \multirow[b]{2}{*}{$S D$} & \multirow[b]{2}{*}{$\begin{array}{c}\text { Mean } \\
\text { Difference }\end{array}$} & \multirow[b]{2}{*}{$t(47)$} & \multirow[b]{2}{*}{$p$} & \multicolumn{2}{|c|}{ CI 95\% } \\
\hline & & & & & & $L L$ & $\overline{U L}$ \\
\hline hr_reactivity* & 5,057 & 18,149 & 5,05793 & 1,931 &, 060 &,- 212 & 10,327 \\
\hline vc_reactivity* & 1,520 & 21,479 & 1,52083 & ,491 & ,626 & $-4,716$ & 7,757 \\
\hline hr_reactivity** & 4,937 & 17,544 & 4,937 & 1,950 &, 057 &,- 157 & 10,031 \\
\hline vc_reactivity** &,- 645 & 15,857 &,- 645 &,- 282 & ,779 & $-5,250$ & 3,958 \\
\hline
\end{tabular}

\section{Challenge and Threat Indexes}

Table 6

Anova one-way for the game task and only for presence condition. CO as dependent measure.

\begin{tabular}{crrrrr}
\hline & \multicolumn{1}{c}{ SS } & df & MS & \multicolumn{1}{c}{ F } & \multicolumn{1}{c}{$\mathrm{p}$} \\
\hline Corrected Model & $16,899^{\mathrm{a}}$ & 2 & 8,449 & 1,931 &, 156 \\
Intercept & 6,709 & 1 & 6,709 & 1,534 &, 222 \\
CO_Baseline & 4,189 & 1 & 4,189 &, 958 &, 333 \\
Demands & 15,954 & 1 & 15,954 & 3,647 &, 062 \\
Error & 205,610 & 47 & 4,375 & & \\
Total & 227,141 & 50 & & & \\
Corrected Total & 222,508 & 49 & & &
\end{tabular}


Table 7

Descriptive statistics of the Main effect of demands

\begin{tabular}{crc}
\hline Condition & $M$ & $S D$ \\
\hline Demanding &,- 26 & .42 \\
Non-Demanding &, 92 & .44 \\
\hline
\end{tabular}

Table 8

Anova one-way for the game task only for presence condition. TPR as dependent measure.

\begin{tabular}{crrrrr}
\hline & \multicolumn{1}{c}{ SS } & df & MS & F & \multicolumn{1}{c}{ p } \\
\hline Corrected Model & $4,470 \mathrm{E} 6$ & 2 & 2234891,028 & 3,550 &, 037 \\
Intercept & 2752814,957 & 1 & 2752814,957 & 4,373 &, 042 \\
Demands & 3168143,318 & 1 & 3168143,318 & 5,032 &, 030 \\
Error & $2,959 \mathrm{E} 7$ & 47 & 629544,400 & & \\
Total & $3,423 \mathrm{E} 7$ & 50 & & & \\
Corrected Total & $3,406 \mathrm{E} 7$ & 49 & & & \\
\hline
\end{tabular}

Table 9

Descriptive statistics of the Main effect of Demands

\begin{tabular}{crc}
\hline Condition & $M$ & $S D$ \\
\hline Demanding & 193,450 & 158,722 \\
Non-Demanding & $-331,194$ & 165,471 \\
\hline
\end{tabular}

Table 10

Between-Subjects Anova (2 $x$ 2) for the persuasion task only for presence condition. CO as dependent measure.

\begin{tabular}{crrrrr}
\hline & \multicolumn{1}{c}{ SS } & df & \multicolumn{1}{c}{ MS } & \multicolumn{1}{c}{ F } & \multicolumn{1}{l}{ p } \\
\hline Corrected Model & $46,544^{\mathrm{a}}$ & 4 & 11,636 & 1,815 &, 143 \\
Intercept &, 718 & 1 &, 718 &, 112 &, 740 \\
CO_Base &, 596 & 1 &, 596 &, 093 &, 762 \\
Demands & 24,845 & 1 & 24,845 & 3,874 &, 055 \\
Arg. Quality & 9,469 & 1 & 9,469 & 1,477 &, 231 \\
Demands * Qual & 6,809 & 1 & 6,809 & 1,062 &, 308 \\
Error & 288,575 & 45 & 6,413 & & \\
Total & 368,020 & 50 & & & \\
Corrected Total & 335,120 & 49 & & & \\
\hline
\end{tabular}

Table 11

Descriptive statistics of the Main effect of Demands

\begin{tabular}{crr}
\hline Condition & \multicolumn{1}{c}{$M$} & \multicolumn{1}{c}{$S D$} \\
\hline Demanding &, 131 &, 508 \\
Non-Demanding & 1,617 &, 534 \\
\hline
\end{tabular}


Table 12

Between-Subjects Anova (2 $\left.\begin{array}{lll}2 & 2\end{array}\right)$ for the persuasion task only for presence condition. TPR as dependent measure.

\begin{tabular}{crrrrr}
\hline & \multicolumn{1}{c}{ SS } & df & \multicolumn{1}{c}{ MS } & \multicolumn{1}{c}{ F } & \multicolumn{1}{c}{$\mathrm{p}$} \\
\hline Corrected Model & $5,914 \mathrm{E} 6$ & 4 & 1478386,538 & 1,726 &, 161 \\
Intercept & 354,450 & 1 & 354,450 &, 000 &, 984 \\
CO_Base & 157989,887 & 1 & 157989,887 &, 184 &, 670 \\
Demands & 3778758,512 & 1 & 3778758,512 & 4,411 &, 041 \\
Arg. Quality & 1421969,598 & 1 & 1421969,598 & 1,660 &, 204 \\
Demands * Qual & 571456,994 & 1 & 571456,994 &, 667 &, 418 \\
Error & $3,855 \mathrm{E} 7$ & 45 & 856680,981 & & \\
Total & $4,674 \mathrm{E} 7$ & 50 & & & \\
Corrected Total & $4,446 \mathrm{E} 7$ & 49 & & & \\
\hline
\end{tabular}

Table 13

Descriptive statistics of the Main effect of Demands

\begin{tabular}{ccc}
\hline Condition & $M$ & $S D$ \\
\hline Demanding & 489,075 & 185,520 \\
Non-Demanding & $-90,288$ & 195,223 \\
\hline
\end{tabular}

\section{Attitude-Change Data.}

Table 14

Factor Analysis and Reliability

\begin{tabular}{lrrrr}
\hline \multicolumn{4}{c}{ Loadings } \\
\cline { 2 - 5 } & Loading & \% of Variance & Cumulative \% & \multicolumn{1}{c}{$\alpha$} \\
\hline 1 & 2,429 & 80,971 & 80,971 &, 874 \\
2 &, 339 & 11,311 & 92,282 & \\
3 &, 232 & 7,718 & 100,000 & \\
\hline
\end{tabular}

Extraction Method: Principal Component Analysis.

1 components extracted.

Table 15

Anova (2 $x$ 2)

\begin{tabular}{crrrrr}
\hline & SS & df & MS & F & p \\
\hline Corrected Model & $17,389^{\mathrm{a}}$ & 7 & 2,484 & 1,773 &, 102 \\
Intercept & 5,248 & 1 & 5,248 & 3,746 &, 056 \\
Demands & 1,574 & 1 & 1,574 & 1,123 &, 292 \\
Arg. Quality &, 123 & 1 &, 123 &, 088 &, 767 \\
Presence &, 086 & 1 &, 086 &, 061 &, 805
\end{tabular}




\begin{tabular}{crrrrr} 
Demands * Qual & 2,616 & 1 & 2,616 & 1,868 &, 175 \\
Demands * Presence & 4,462 & 1 & 4,462 & 3,185 &, 078 \\
Quality * Presence &, 650 & 1 &, 650 &, 464 &, 498 \\
Qual * Dem * Pres & 6,826 & 1 & 6,826 & 4,873 &, 030 \\
Error & 126,077 & 90 & 1,401 & & \\
Total & 148,556 & 98 & & & \\
Corrected Total & 143,466 & 97 & & & \\
\hline
\end{tabular}

Table 16

Descriptive statistics of the Demands $x$ Argument quality $x$ Presence Interaction

\begin{tabular}{ccccccccc}
\hline & \multicolumn{9}{c}{ Presence } & \multicolumn{9}{c}{ Alone } \\
\hline \multirow{2}{*}{ Condition } & \multicolumn{2}{c}{ Demanding } & \multicolumn{2}{c}{ Non-Demanding } & \multicolumn{2}{c}{ Demanding } & \multicolumn{2}{c}{ Non-Demanding } \\
\cline { 2 - 10 } & $M$ & $S D$ & $M$ & $M$ & $S D$ & $S D$ & $M$ & $S D$ \\
\hline Strong & 1,077 &, 328 &,- 462 &, 328 &,- 103 &, 328 &, 273 &, 357 \\
Weak &, 128 &, 328 &, 303 &, 357 &, 333 &, 342 &, 306 &, 342 \\
\hline
\end{tabular}

Table 17

Contrasts revealing the impact of the version of the game/motivational state on the differentiation between strong and weak arguments in the presence condition.

\begin{tabular}{|c|c|c|c|c|c|c|c|c|}
\hline \multirow[b]{2}{*}{ Condition } & \multicolumn{2}{|c|}{ Strong Arguments } & \multicolumn{2}{|c|}{ Weak Arguments } & \multirow[b]{2}{*}{$t(26)$} & \multirow[b]{2}{*}{$p$} & \multicolumn{2}{|c|}{ CI 95\% } \\
\hline & $M$ & $S D$ & $M$ & $S D$ & & & $L L$ & $U L$ \\
\hline \multirow[t]{2}{*}{ Demanding } & 1.02 & 1.18 & .10 & .04 & 2.04 & .04 & .03 & 1.87 \\
\hline & & & & & $t(28)$ & & & \\
\hline Non-Demanding & -.49 & 1.19 & .23 & .04 & 1.58 & .12 & -1.13 & .19 \\
\hline
\end{tabular}

Mediation of the Unitary Index on Attitude-Change towards Acid Rain

Table 18

Ancova $(2 \times 2)$

\begin{tabular}{crrrrr}
\hline & \multicolumn{1}{c}{ SS } & df & MS & \multicolumn{1}{l}{ F } & \multicolumn{1}{c}{$\mathrm{p}$} \\
\hline Corrected Model & $17,394^{\mathrm{a}}$ & 8 & 2,174 & 1,535 &, 157 \\
Intercept & 5,252 & 1 & 5,252 & 3,708 &, 057 \\
Unitray Index &, 005 & 1 &, 005 &, 003 &, 955 \\
Demands & 1,518 & 1 & 1,518 & 1,071 &, 303 \\
Arg. Qual. &, 127 & 1 &, 127 &, 089 &, 766 \\
Presence &, 089 & 1 &, 089 &, 063 &, 802 \\
Demands * Qual. & 2,619 & 1 & 2,619 & 1,849 &, 177 \\
Demands * Presence & 4,238 & 1 & 4,238 & 2,992 &, 087 \\
Qual.* Presence &, 653 & 1 &, 653 &, 461 &, 499 \\
Dem.* Qual * Pres & 6,357 & 1 & 6,357 & 4,488 &, 037 \\
Error & 126,072 & 89 & 1,417 & &
\end{tabular}




\begin{tabular}{ccc} 
Total & 148,556 & 98 \\
Corrected Total & 143,466 & 97 \\
\hline
\end{tabular}

Mediation of Cognitive evaluations on Attitude-Change towards Acid Rain

Table 19

Ancova (2 $\times 2$ 2)

\begin{tabular}{crrrrr}
\hline & \multicolumn{1}{c}{ SS } & df & MS & \multicolumn{1}{l}{ F } & \multicolumn{1}{c}{$\mathrm{p}$} \\
\hline Corrected Model & $23,265^{\text {a }}$ & 8 & 2,908 & 2,169 &, 038 \\
Intercept & 7,195 & 1 & 7,195 & 5,366 &, 023 \\
Cogn. Evaluations & 5,359 & 1 & 5,359 & 3,996 &, 049 \\
Demands &, 020 & 1 &, 020 &, 015 &, 902 \\
Quality &, 025 & 1 &, 025 &, 018 &, 892 \\
Presence &, 739 & 1 &, 739 &, 551 &, 460 \\
Demands * Qual. & 3,066 & 1 & 3,066 & 2,287 &, 134 \\
Demands * Presence & 5,495 & 1 & 5,495 & 4,098 &, 046 \\
Qual.* Presence &, 727 & 1 &, 727 &, 542 &, 463 \\
Dem.* Qual * Pres & 4,025 & 1 & 4,025 & 3,002 &, 087 \\
Error & 115,324 & 86 & 1,341 & & \\
Total & 142,000 & 95 & & & \\
Corrected Total & 138,589 & 94 & & & \\
\hline
\end{tabular}

\section{Mediation of Mood on Attitude-Change towards Acid Rain}

Table 20

Ancova (2 $\times 2)$

\begin{tabular}{crrrrr}
\hline & \multicolumn{1}{c}{ SS } & df & \multicolumn{1}{c}{ MS } & \multicolumn{1}{c}{ F } & \multicolumn{1}{c}{ p } \\
\hline Corrected Model & $16,242^{\mathrm{a}}$ & 8 & 2,030 & 1,426 &, 197 \\
Intercept &, 204 & 1 &, 204 &, 144 &, 706 \\
Mood &, 004 & 1 &, 004 &, 003 &, 958 \\
Presence &, 178 & 1 &, 178 &, 125 &, 725 \\
Demands & 1,830 & 1 & 1,830 & 1,285 &, 260 \\
Quality &, 284 & 1 &, 284 &, 200 &, 656 \\
Presence * Demands & 3,456 & 1 & 3,456 & 2,428 &, 123 \\
Presence * Qual. &, 867 & 1 &, 867 &, 609 &, 437 \\
Demands * Qual & 2,950 & 1 & 2,950 & 2,072 &, 154 \\
Pres * Dem * Qual & 5,404 & 1 & 5,404 & 3,795 &, 055 \\
Error & 123,864 & 87 & 1,424 & & \\
Total & 144,000 & 96 & & & \\
Corrected Total & 140,106 & 95 & & & \\
\hline
\end{tabular}




\section{Appendix I: Statistics of Experiment 4}

\section{Effectiveness of the familiarity manipulation.}

Table 1

Between-Subjects Anova (2 $x 2 \times 2)$

\begin{tabular}{crrrrr}
\hline & \multicolumn{1}{c}{ SS } & df & \multicolumn{1}{c}{ MS } & \multicolumn{1}{c}{ F } & \multicolumn{1}{c}{ p } \\
\hline Corrected Model & $65,204^{\mathrm{a}}$ & 7 & 9,315 & 3,018 &, 008 \\
Intercept & 1364,580 & 1 & 1364,580 & 442,098 &, 000 \\
Presence & 9,889 & 1 & 9,889 & 3,204 &, 077 \\
Repetition & 38,058 & 1 & 38,058 & 12,330 &, 001 \\
Quality &, 520 & 1 &, 520 &, 168 &, 683 \\
Presence * Repetition & 1,307 & 1 & 1,307 &, 423 &, 517 \\
Presence * Quality &, 453 & 1 &, 453 &, 147 &, 703 \\
Repetition* Quality & 3,929 & 1 & 3,929 & 1,273 &, 263 \\
Pres * Rep * Qual & 7,240 & 1 & 7,240 & 2,346 &, 130 \\
Error & 231,495 & 75 & 3,087 & & \\
Total & 1665,000 & 83 & & & \\
Corrected Total & 296,699 & 82 & & & \\
\hline
\end{tabular}

Table 2

Descriptive statistics of the main effect of the Repetition

\begin{tabular}{ccc}
\hline Condition & $M$ & $S D$ \\
\hline No-Repetition & 3,389 &, 276 \\
Repetition & 4,748 &, 271 \\
\hline
\end{tabular}

\section{Atitude-Change Data: Weight Loss Centre Message}

Table 3

Factor Analysis and Reliability

\begin{tabular}{lrrrr}
\hline \multicolumn{4}{c}{ Loadings } \\
\cline { 2 - 5 } & Loading & \% of Variance & Cumulative \% & \multicolumn{1}{c}{$\alpha$} \\
\hline 1 & 2,174 & 72,472 & 72,472 &, 806 \\
2 &, 523 & 17,449 & 89,921 & \\
3 &, 302 & 10,079 & 100,000 & \\
\hline
\end{tabular}

Extraction Method: Principal Component Analysis.

1 components extracted.

Table 4

Between-Subjects Anova (2 x 2) 


\begin{tabular}{crrrrr}
\hline & \multicolumn{1}{c}{ SS } & df & \multicolumn{1}{c}{ MS } & \multicolumn{1}{c}{ F } & \multicolumn{1}{c}{$\mathrm{p}$} \\
\hline Corrected Model & $13,357^{\text {a }}$ & 7 & 1,908 &, 983 &, 453 \\
Intercept & 95,759 & 1 & 95,759 & 49,308 &, 000 \\
Presence & 4,869 & 1 & 4,869 & 2,507 &, 119 \\
Repetition &, 105 & 1 &, 105 &, 054 &, 817 \\
Quality & 4,364 & 1 & 4,364 & 2,247 &, 139 \\
Presence * Repetition &, 174 & 1 &, 174 &, 090 &, 766 \\
Presence * Quality & 2,268 & 1 & 2,268 & 1,168 &, 284 \\
Repetition* Quality &, 001 & 1 &, 001 &, 000 &, 983 \\
Pres * Rep * Qual & 1,877 & 1 & 1,877 &, 966 &, 330 \\
Error & 108,754 & 56 & 1,942 & & \\
Total & 215,556 & 64 & & & \\
Corrected Total & 122,111 & 63 & & & \\
\hline
\end{tabular}

\section{Atitude-Change Data: Acid Rain Message}

Table 5

Factor Analysis

\begin{tabular}{lrrrr}
\hline \multicolumn{4}{c}{ Loadings } \\
\cline { 2 - 5 } & Loading & \% of Variance & Cumulative \% & \multicolumn{1}{c}{$\alpha$} \\
\hline 1 & 1,693 & 56,422 & 56,422 &, 604 \\
2 &, 852 & 28,403 & 84,825 & \\
3 &, 455 & 15,175 & 100,000 & \\
\hline
\end{tabular}

Extraction Method: Principal Component Analysis.

1 components extracted.

Table 6

Between-Subjects Anova $(2 \times 2 \times 2)$

\begin{tabular}{crrrrr}
\hline & \multicolumn{1}{c}{ SS } & df & \multicolumn{1}{c}{ MS } & \multicolumn{1}{c}{ F } & \multicolumn{1}{c}{$\mathrm{p}$} \\
\hline Corrected Model & $27,786^{\mathrm{a}}$ & 7 & 3,969 & 2,816 &, 012 \\
Intercept & 40,726 & 1 & 40,726 & 28,892 &, 000 \\
Presence &, 026 & 1 &, 026 &, 019 &, 892 \\
Repetition &, 204 & 1 &, 204 &, 145 &, 705 \\
Quality & 5,467 & 1 & 5,467 & 3,879 &, 053 \\
Presence * Repetition & 14,811 & 1 & 14,811 & 10,507 &, 002 \\
Presence * Quality & 1,903 & 1 & 1,903 & 1,350 &, 249 \\
Repetition* Quality & 4,289 & 1 & 4,289 & 3,043 &, 085 \\
Pres * Rep * Qual &, 293 & 1 &, 293 &, 208 &, 650 \\
Error & 105,720 & 75 & 1,410 & & \\
Total & 176,533 & 83 & & & \\
Corrected Total & 133,506 & 82 & & & \\
\hline
\end{tabular}


Table 7

Descriptive statistics of the Argument Quality x Repetition Interaction

\begin{tabular}{ccccc}
\hline & \multicolumn{2}{c}{ No-repetition } & \multicolumn{2}{c}{ Repetition } \\
\cline { 2 - 5 } Condition & $M$ & $S D$ & $M$ & $S D$ \\
\hline Weak &, 267 &, 265 &, 624 &, 253 \\
Strong & 1,238 &, 262 &, 682 &, 265 \\
\hline
\end{tabular}

Table 8

Contrasts revealing the impact of the familiarity on the differentiation between strong and weak arguments in attitude-change.

\begin{tabular}{|c|c|c|c|c|c|c|c|c|}
\hline \multirow[b]{2}{*}{ Condition } & \multicolumn{2}{|c|}{ Strong Arguments } & \multicolumn{2}{|c|}{ Weak Arguments } & \multirow[b]{2}{*}{$t(41)$} & \multirow[b]{2}{*}{$p$} & \multicolumn{2}{|c|}{ CI 95\% } \\
\hline & $M$ & $S D$ & $M$ & $S D$ & & & $L L$ & $U L$ \\
\hline No-Repetition & 1.21 & .21 & .22 & .09 & 2.60 & .01 & .23 & 1.71 \\
\hline Repetition & .69 & .10 & .61 & .11 & .16 & .88 & -.67 & .78 \\
\hline
\end{tabular}

\section{Atitude-Change Data: Number of Thoughts}

Table 9

Between-Subjects Anova (2 x 2)

\begin{tabular}{crrrrr}
\hline & \multicolumn{1}{c}{ SS } & df & \multicolumn{1}{c}{ MS } & \multicolumn{1}{c}{ F } & \multicolumn{1}{c}{$\mathrm{p}$} \\
\hline Corrected Model & $27,849^{\mathrm{a}}$ & 7 & 3,978 & 3,449 &, 004 \\
Intercept & 355,577 & 1 & 355,577 & 308,237 &, 000 \\
Presence & 1,596 & 1 & 1,596 & 1,383 &, 244 \\
Repetition & 5,706 & 1 & 5,706 & 4,946 &, 030 \\
Quality & 4,760 & 1 & 4,760 & 3,766 &, 051 \\
Presence * Repetition & 8,915 & 1 & 8,915 & 7,728 &, 007 \\
Presence * Quality &, 211 & 1 &, 211 &, 183 &, 670 \\
Repetition * Quality & 2,659 & 1 & 2,659 & 2,305 &, 134 \\
Pres * Rep * Qual & 1,596 & 1 & 1,596 & 1,383 &, 244 \\
Error & 71,522 & 64 & 1,154 & & \\
Total & 456,000 & 70 & & & \\
Corrected Total & 99,371 & 69 & & & \\
\hline
\end{tabular}

Table 10

Descriptive statistics of the Main effect of Repetition

\begin{tabular}{ccc}
\hline Condition & $M$ & $S D$ \\
\hline No-Repetition & 2,569 &, 190 \\
Repetition & 1,992 &, 177 \\
\hline
\end{tabular}


Table 11

Contrasts revealing the different number of thoughts as a function of repetition and no-repetition in the presence conditions.

\begin{tabular}{cccccccccc}
\hline & \multicolumn{2}{c}{ Presence } & & \multicolumn{2}{c}{ Alone } & & & \multicolumn{2}{c}{$C I 95 \%$} \\
\cline { 2 - 6 } \cline { 8 - 10 } Condition & $M$ & $S D$ & & $M$ & $S D$ & $t(41)$ & $p$ & $L L$ & $U L$ \\
\hline No-Repetition & 3.08 & .28 & & 2.06 & .25 & 2.21 & .03 & .08 & 1.51 \\
\hline Repetition & 1.78 & .25 & & 2.20 & .26 & .68 & .49 & .94 & .46 \\
\hline
\end{tabular}

\section{Atitude-Change Data: Type of Thoughts}

Table 12

Mixed Anova (2 $x 2 \times$ 2), Type of Thoughts as Repeated Measure.

\begin{tabular}{ccrrrr}
\hline & SS & Df & MS & \multicolumn{1}{c}{ F } & \multicolumn{1}{c}{ p } \\
\hline Type of Thought &, 022 & 1 &, 022 &, 111 &, 740 \\
T.Thought * Presence &, 004 & 1 &, 004 &, 018 &, 892 \\
T.Thought * Repetition &, 038 & 1 &, 038 &, 193 &, 662 \\
T.Thought * Arg. Quality & 1,148 & 1 & 1,148 & 5,756 &, 019 \\
T.Thought * Pres * Rep &, 189 & 1 &, 189 &, 945 &, 334 \\
T.Thought * Pres * A.Qual &, 083 & 1 &, 083 &, 418 &, 520 \\
T.Thought * Rep * A. Qual &, 529 & 1 &, 529 & 2,650 &, 108 \\
T.Thought * Pres * Rep * &, 061 & 1 &, 061 &, 308 &, 581 \\
Arg.Quality & & & & & \\
\hline
\end{tabular}

Table 13

Descriptive statistics of the TypeThought $x$ Repetition Interaction

\begin{tabular}{ccccc}
\hline & \multicolumn{2}{c}{ No-repetition } & \multicolumn{2}{c}{ Repetition } \\
\cline { 2 - 5 } Condition & $M$ & $S D$ & $M$ & $S D$ \\
\hline Favorable & .309 & .059 & .272 & .059 \\
Unfavorable & .301 & .066 & .329 & .065 \\
\hline
\end{tabular}

Table 14

Descriptive statistics of the Type of thoughts $x$ Repetition $x$ Argument quality

\begin{tabular}{ccccccccc}
\hline & \multicolumn{1}{l}{ No-Repetition } & \multicolumn{2}{c}{ Repetition } \\
\hline \multirow{2}{*}{ Condition } & \multicolumn{2}{c}{ Strong } & \multicolumn{2}{c}{ Weak } & \multicolumn{2}{c}{ Strong } & \multicolumn{2}{c}{ Weak } \\
\cline { 2 - 10 } & $M$ & $S D$ & $M$ & $M$ & $S D$ & $S D$ & $M$ & $S D$ \\
\hline Favorable &, 472 &, 079 &, 146 &, 088 &, 241 &, 085 &, 304 &, 081 \\
Unfavorable &, 170 &, 088 &, 432 &, 097 &, 241 &, 094 &, 417 &, 090 \\
\hline
\end{tabular}


Table 15

Contrasts revealing the difference in the number of favourable thoughts as a function of message quality and familiarity conditions.

\begin{tabular}{|c|c|c|c|c|c|c|c|c|}
\hline \multirow[b]{2}{*}{ Condition } & \multicolumn{2}{|c|}{ Strong Arguments } & \multicolumn{2}{|c|}{ Weak Arguments } & \multirow[b]{2}{*}{$t(38)$} & \multirow[b]{2}{*}{$p$} & \multicolumn{2}{|c|}{ CI 95\% } \\
\hline & $M$ & $S D$ & $M$ & $S D$ & & & $L L$ & $U L$ \\
\hline Repetition & .24 & .08 & .30 & .08 & 2.83 & .0001 & .17 & 1.01 \\
\hline No-Repetition & .47 & .08 & .15 & .09 & .55 & .59 & -.29 & .52 \\
\hline
\end{tabular}

$\mathrm{CI}=$ Confidence interval $(95 \%) ; \mathrm{LL}=$ lower limit; $\mathrm{UL}=$ upper limit

\section{Atitude-Change Data: Mediation analyses}

Table 16

Regression analyses in the no-repetition condition

\begin{tabular}{|c|c|c|c|c|c|}
\hline \multirow[b]{2}{*}{ Predictor* } & \multicolumn{2}{|c|}{$\begin{array}{c}\text { Unstandardized } \\
\text { Coefficients } \\
\end{array}$} & \multirow[t]{2}{*}{$\begin{array}{c}\text { Standardized } \\
\text { Coefficients }\end{array}$} & \multirow[t]{2}{*}{$t$} & \multirow[t]{2}{*}{ Sig. } \\
\hline & $B$ & $S D$ & & & \\
\hline Arg. Quality & 1,021 & ,352 & ,421 & 2,901 & ,006 \\
\hline Arg. Quality ** & 1,366 & ,593 & ,541 & 2,306 & ,029 \\
\hline Thought Index &,- 442 & ,528 &,- 159 &,- 837 & ,410 \\
\hline
\end{tabular}

* Attitude-Change towards Acid Rain as Dependent Measure

** Thought Index constant

Table 17

Regression analyses in the repetition condition

\begin{tabular}{cccccc}
\hline & \multicolumn{2}{c}{$\begin{array}{c}\text { Unstandardized } \\
\text { Coefficients }\end{array}$} & $\begin{array}{c}\text { Standardized } \\
\text { Coefficients }\end{array}$ & $t$ & Sig. \\
\cline { 2 - 6 } Predictor* & $B$ & $S D$ &, 022 &, 141 &, 888 \\
\hline Arg. Quality &, 059 &, 417 &,- 055 &,- 296 &, 769 \\
Arg. Quality ** &,- 137 &, 463 &, 106 &, 574 &, 571 \\
Thought Index &, 250 &, 436 & & \\
\hline * Attitude-Change towards Acid Rain as Dependent Measure \\
** Thought Index constant
\end{tabular}

\section{Challenge and Threat Data: Baseline Differences}

Table 18

Between-Subjects Manova (2 x 2 ×2)

\begin{tabular}{crrrrr}
\hline & Value* & F & $\begin{array}{r}\text { Hypothesis } \\
\text { df }\end{array}$ & Error df & Sig. \\
\hline Presence &, 933 & $1,255^{\mathrm{a}}$ & 4,000 & 70,000 &, 296 \\
Repetition &, 965 &, $629^{\mathrm{a}}$ & 4,000 & 70,000 &, 643 \\
Argument Quality &, 929 & $1,336^{\mathrm{a}}$ & 4,000 & 70,000 &, 265 \\
Presence x Repetition &, 911 & $1,711^{\mathrm{a}}$ & 4,000 & 70,000 &, 157 \\
Presence x Arg. Quality &, 963 &, $670^{\mathrm{a}}$ & 4,000 & 70,000 &, 615
\end{tabular}




\begin{tabular}{crrrrr} 
Repetition x Arg. Quality &, 939 & $1,139^{\mathrm{a}}$ & 4,000 & 70,000 &, 346 \\
Pres * Rep * Arg. Qual. &, 932 & $1,276^{\mathrm{a}}$ & 4,000 & 70,000 &, 288 \\
\hline
\end{tabular}

*Wilks' Lambda.

\section{Challenge and Threat Data: Task-Engagement}

Table 19

T-tests for hr and vc against zero for the presence condition.

\begin{tabular}{|c|c|c|c|c|c|c|c|}
\hline \multirow[b]{2}{*}{ Condition } & \multirow[b]{2}{*}{$M$} & \multirow[b]{2}{*}{$S D$} & \multirow[b]{2}{*}{$\begin{array}{c}\text { Mean } \\
\text { Difference } \\
\end{array}$} & \multirow[b]{2}{*}{$t(49)$} & \multirow[b]{2}{*}{$p$} & \multicolumn{2}{|c|}{ CI 95\% } \\
\hline & & & & & & $L L$ & $U L$ \\
\hline hr_reactivity & 10.24 & 22.89 & 10.24 & 3.16 & .003 & $-1,319$ & 17,487 \\
\hline vc_reactivity & 4.95 & 16.09 & 4.93 & 2.17 & .04 & 5,295 & 22,258 \\
\hline
\end{tabular}

$\mathrm{CI}=$ Confidence interval (95\%); $\mathrm{LL}=$ lower limit; $\mathrm{UL}=$ upper limit

Table 20

T-tests for hr and vc against zero for the alone condition.

\begin{tabular}{|c|c|c|c|c|c|c|c|}
\hline \multirow[b]{2}{*}{ Condition } & \multirow[b]{2}{*}{$M$} & \multirow[b]{2}{*}{$S D$} & \multirow[b]{2}{*}{$\begin{array}{c}\text { Mean } \\
\text { Difference } \\
\end{array}$} & \multirow[b]{2}{*}{$t(42)$} & \multirow[b]{2}{*}{$p$} & \multicolumn{2}{|c|}{ CI 95\% } \\
\hline & & & & & & $L L$ & $U L$ \\
\hline hr_reactivity & 8.35 & 27.48 & 8.35 & 1.99 & .06 &,- 107 & 16,804 \\
\hline vc_reactivity & 4.59 & 23.76 & 4.59 & 1.27 & .21 & $-2,717$ & 11,907 \\
\hline
\end{tabular}

\section{Challenge and Threat Indexes}

Table 21

Between-Subjects Anova (2 × 2), CO as dependent measure

\begin{tabular}{crrrrr}
\hline & \multicolumn{1}{c}{ SS } & df & \multicolumn{1}{c}{ MS } & \multicolumn{1}{c}{ F } & \multicolumn{1}{c}{$\mathrm{p}$} \\
\hline Corrected Model & $169,733^{\mathrm{a}}$ & 3 & 56,578 & 1,917 &, 144 \\
Intercept &, 253 & 1 &, 253 &, 009 &, 927 \\
Repetition & 141,853 & 1 & 141,853 & 4,807 &, 035 \\
Arg. Quality & 25,732 & 1 & 25,732 &, 872 &, 357 \\
Rep * Arg. Qual &, 800 & 1 &, 800 &, 027 &, 870 \\
Error & 1062,314 & 36 & 29,509 & & \\
Total & 1232,765 & 40 & & & \\
Corrected Total & 1232,047 & 39 & & & \\
\hline
\end{tabular}


Table 22

Descriptive statistics of the Main effect of Repetition

\begin{tabular}{ccc}
\hline Condition & $M$ & $S D$ \\
\hline No-Repetition & $-1,808$ & 1,248 \\
Repetition & 1,968 & 1,187 \\
\hline
\end{tabular}

Table 23

Between-Subjects Anova (2 $\times 2)$, TPR as dependent measure

\begin{tabular}{crrrrr}
\hline & \multicolumn{1}{c}{ SS } & df & \multicolumn{1}{c}{ MS } & \multicolumn{1}{c}{ F } & \multicolumn{1}{c}{$\mathrm{p}$} \\
\hline Corrected Model & $2,506 \mathrm{E} 6$ & 3 & 835376,020 & 1,823 &, 160 \\
Intercept & 5758,990 & 1 & 5758,990 &, 013 &, 911 \\
Repetition & 2467491,352 & 1 & 2467491,352 & 5,384 &, 026 \\
Argument Qual & 41667,680 & 1 & 41667,680 &, 091 &, 765 \\
Fam * Qual & 10009,496 & 1 & 10009,496 &, 022 &, 883 \\
Error & $1,650 \mathrm{E} 7$ & 36 & 458295,423 & & \\
Total & $1,903 \mathrm{E} 7$ & 40 & & & \\
Corrected Total & $1,900 \mathrm{E} 7$ & 39 & & & \\
\hline
\end{tabular}

Table 24

Descriptive statistics of the Main effect of Repetition

\begin{tabular}{ccc}
\hline Condition & $M$ & $S D$ \\
\hline No-Repetition & 236,966 & 155,524 \\
Repetition & $-261,025$ & 147,896 \\
\hline
\end{tabular}

Mediation of Unitary Index on Attitude-Change towards Acid Rain

Table 25

Ancova $(2 \times 2)$

\begin{tabular}{crrrrr}
\hline & \multicolumn{1}{c}{ SS } & df & \multicolumn{1}{c}{ MS } & \multicolumn{1}{c}{ F } & \multicolumn{1}{c}{$\mathrm{p}$} \\
\hline Corrected Model & $9,991^{\text {a }}$ & 4 & 2,498 & 1,507 &, 209 \\
Intercept & 46,191 & 1 & 46,191 & 27,875 &, 000 \\
Index_CT &, 122 & 1 &, 122 &, 074 &, 787 \\
Repetition & 1,061 & 1 & 1,061 &, 640 &, 426 \\
Arg. Quality & 4,624 & 1 & 4,624 & 2,791 &, 099 \\
Rep. * Arg. Qual. & 4,436 & 1 & 4,436 & 2,677 &, 106 \\
Error & 115,998 & 70 & 1,657 & & \\
Total & 173,001 & 75 & & & \\
Corrected Total & 125,988 & 74 & & & \\
\hline
\end{tabular}




\section{Mediation of Cognitive evaluations on Attitude-Change towards Acid Rain}

Table 26

Ancova (2 $x$ 2)

\begin{tabular}{crrrrr}
\hline & \multicolumn{1}{c}{ SS } & df & \multicolumn{1}{c}{ MS } & \multicolumn{1}{c}{ F } & \multicolumn{1}{c}{$\mathrm{p}$} \\
\hline Corrected Model & $33,243^{\mathrm{a}}$ & 8 & 4,155 & 2,995 &, 006 \\
Intercept &, 373 & 1 &, 373 &, 269 &, 605 \\
Cog Evaluation & 5,012 & 1 & 5,012 & 3,613 &, 061 \\
Repetition &, 238 & 1 &, 238 &, 172 &, 680 \\
Arg. Quality & 7,645 & 1 & 7,645 & 5,511 &, 022 \\
Rep. * Arg. Qual. & 4,072 & 1 & 4,072 & 2,935 &, 091 \\
Error & 99,886 & 72 & 1,387 & & \\
Total & 174,246 & 81 & & & \\
Corrected Total & 133,129 & 80 & & & \\
\hline
\end{tabular}

\section{Mediation of Mood on Attitude-Change towards Acid Rain}

\section{Table 27}

Ancova (2 $\times 2)$

\begin{tabular}{crrrrr}
\hline & \multicolumn{1}{c}{ SS } & df & MS & \multicolumn{1}{l}{ F } & \multicolumn{1}{l}{ p } \\
\hline Corrected Model & $11,701^{\mathrm{a}}$ & 4 & 2,925 & 1,807 &, 136 \\
Intercept & 2,466 & 1 & 2,466 & 1,523 &, 221 \\
Mood &, 183 & 1 &, 183 &, 113 &, 738 \\
Repetition &, 577 & 1 &, 577 &, 356 &, 552 \\
Arg. Quality & 5,701 & 1 & 5,701 & 3,521 &, 064 \\
Rep. * Arg. Qual. & 5,038 & 1 & 5,038 & 3,111 &, 082 \\
Error & 121,427 & 75 & 1,619 & & \\
Total & 173,797 & 80 & & & \\
Corrected Total & 133,127 & 79 & & & \\
\hline
\end{tabular}

